UAB-FT-369

PLY-MS-95-03

\title{
CONSTITUENT QUARKS FROM QCD
}

\author{
MARTin LAVELLE \\ Grup de Física Teòrica and IFAE \\ Edificio $\mathrm{Cn}$ \\ Universitat Autónoma de Barcelona \\ E-08193 Bellaterra (Barcelona) \\ Spain \\ (e-mail: lavelle@ifae.es) \\ AND \\ David McMullan \\ School of Mathematics and Statistics \\ University of Plymouth \\ Drake Circus, Plymouth, Devon PL4 8AA \\ U.K. \\ (e-mail: d.mcmullan@plymouth.ac.uk)
}

\begin{abstract}
Starting from the observation that colour charge is only well defined on gauge invariant states, we construct perturbatively gauge invariant, dynamical dressings for individual quarks. Explicit calculations show that an infra-red finite mass-shell renormalisation of the gauge invariant, dressed propagator is possible and, further, that operator product effects, which generate a running mass, may be included in a gauge invariant way in the propagator. We explain how these fields may be combined to form hadrons and show how the interquark potential can now be directly calculated. The onset of confinement is identified with an obstruction to building a non-perturbative dressing. We propose several methods to extract the hadronic scale from the interquark potential. Various extensions are discussed.
\end{abstract}

Physics Reports C279 (1997) 1 


\section{Introduction}

More than twenty years after the birth of Quantum Chromodynamics (QCD) it seems fair to say that physicists are convinced that QCD is the correct theory of the strong interactions $^{[1]}$. The successes of Chromodynamics are many: the $Q^{2}$ dependence of structure functions at high $Q^{2}$ shows the scaling violations that QCD predicts and the study of the long expected quark and gluon jets now forms a sub-field of the physics of strong interactions. Moving down to lower energies, the somewhat approximate approach of QCD sum rules has predicted many hadronic parameters, such as masses, rather well. Many phenomenological models have also received some input from the insights spawned by QCD. This theory is so far fully supported by experiment.

And yet despite the many successes of the theory much, if not most, of low energy hadronic physics cannot be extracted directly from QCD. The most visible problem of the theory being the non-observation of the fundamental QCD particles: the quarks and gluons. This has led to the dogma of confinement which teaches that quarks and gluons are permanently confined inside colour singlets - the observed hadrons. Another problem is our inability to calculate the structure functions themselves (in contrast to their $Q^{2}$ dependence) and, more recently, there has been controversy about how the spin of the proton is produced ${ }^{[1]}$.

Quarks were initially introduced as constituent particles so as to introduce a semblance of order into the "particle zoo" ${ }^{[2,3]}$. Mesons are in this picture made up of a quark and an antiquark, and baryons of three quarks. The hadrons can then be fitted into multiplets and their spectrum understood. In this way new particles were even predicted ${ }^{[4]}$. Later a colour quantum number was introduced to explain the statistics of the baryons. Deep inelastic scattering experiments ${ }^{[5]}$ showed that there were seemingly freely moving objects inside the nucleus and these 'partons' were identified with the naive quarks of the quark model. The discovery ${ }^{[6]}$ of asymptotic freedom showed that in non-abelian gauge theories the coupling grows small at short distances and the quarks of QCD were then identified with the partons.

QCD is a non-abelian gauge theory modelled upon Quantum Electrodynamics (QED). The interaction between the quarks is carried by massless vector (gauge) bosons, the gluons. As is well known a decisive difference between QED and QCD is that the gluons also carry QCD (colour) charges and the quarks feel the colour charges of the gluons. It is, however, 
not possible to directly identify the quarks of the QCD Lagrangian with the constituent quarks which explain hadronic spectroscopy. The most obvious reason for this is the fact that the masses of the QCD Lagrangian quarks are, generally speaking, much smaller than typical hadronic mass scales. (Three times the Lagrangian mass of the $u$ quark is only about $1 \%$ of the mass of the nucleon.) In addition, deep inelastic scattering experiments show that about half the momentum of the nucleon is carried by particles which, unlike quarks, do not have an electric charge. These are believed to be the gluons. It is also seen that nucleons contain, as well as the three valence quarks which account for quantum numbers such as baryon number and electromagnetic charge, quark-antiquark pairs — the sea quarks. The interpretation of deep inelastic scattering, it should be further stressed, relies upon the quarks having small masses. We see that although quark fields are now more solidly rooted, in the theory of QCD, we have lost touch with the initial motivation for their introduction, viz. hadronic spectroscopy.

The success of QCD together with that of the spectroscopic description of hadrons in terms of more massive, constituent quarks leads in fact to our modern picture of hadrons, where the three valence quarks of the nucleon, say, are dressed with gluons and quarkantiquark pairs in such a way that they become much heavier. Although the very language being used leads to an appealing picture of this dressing as a swirling cloud of glue and sea-quarks, the precise nature of the dressing is, to say the least, hard to pin down within the context of QCD. This dressing, with colour charged objects, has in the first instance to explain the successes of the constituent quark model. As such it has to be constructed in such a way that the quantum numbers of the hadrons are still produced. Insofar as the constituent quark picture applies, one expects quasiparticle type constituent quarks to also have quantum numbers such as colour charges and spin. In addition, this dressing must be such that it is compatible with the confinement of coloured objects.

What is required is a clear physical reason for the need for such a dressing in QCD. Our view is that if QCD is the correct description of strong interactions then its structure should restrict the dressing so as to conform with the above observed properties. The main aim of this paper is to investigate the guiding principles behind the process of dressing such charged fields in a gauge theory. In particular, we will discuss why one in fact must dress quark (and gluonic) fields and how far this dressing may be taken. We will argue that these results point to a systematic approach to dressing both quarks and gluons that make it at least plausible that a constituent quark picture can emerge from QCD. 
Our first motivation for dressing the Lagrangian quarks is to note that they are not gauge invariant. The gauge symmetry shows that not all fields are physically significant. To produce a gauge invariant quark field it is necessary to dress the fermions with coloured gluons. In QED electrons must also be dressed with photons, as will be discussed below, but a major difference is that photons are not electrically charged, in contrast to the colour charged gluons. We will, however, see that the gluonic dressing is such that it actually gives a quark a well defined colour charge. Since we want to use the standard tools of theoretical physics, our approach to dressing quarks will be perturbative. We will give explicit dressings to low orders in the coupling and then show how they may be systematised.

One views dressing as surrounding the charged particle with a cloud of gauge fields. We will see that, at least in this perturbative description, this cloud spreads out over the whole space, resulting in a highly non-local structure. This is not unexpected in $\mathrm{QED}^{[7]}$, but in QCD we will see that there is a non-perturbative obstruction to the construction of this dressing ${ }^{[8]}$. This breakdown of the quark description should be identified with confinement. We cannot in the non-perturbative domain talk about quark fields. This is to be contrasted with the situation in both QED and spontaneously broken non-abelian gauge theories (i.e., with Higgs' scalars) where such problems do not arise ${ }^{[9]}$.

As well as gauge invariance, dressing is necessary because use of, for example, the free electron as an asymptotic field in QED leads to infra-red divergences (see, for example, the summary in Supplement 4 of Ref. 10). Essentially the long range of the Coulomb force, which only falls off as $1 / R$, is responsible for this ${ }^{[11,12]}$. In QCD the infra-red problems seem more severe. We will argue, and also show by explicit calculation, that the gauge invariant dressing can remove the infra-red problems.

A physically quite reasonable, but at first sight surprising, aspect of this dressing is that it depends upon the velocity of the quark. In the context of QED this is simply a reflection of the fact that the electric and magnetic fields of a charged particle vary with its velocity. We will demonstrate that this non-covariance is really unavoidable. A technical consequence of this, though, will be that our formulation will be non-covariant. In conjunction with the non-locality of the dressing, this means that we will need to carefully show that standard field theoretic methods can still be applied to this description of constituent quarks. 
Some parts of the work reported here, and certain closely related topics, have already been reported, albeit in rather terser form, elsewhere ${ }^{[13,14,8,9]}$. However, we have tried to make this paper self-contained. As we have stated above, the basic theme in this approach is to use gauge invariance as the guiding principle in the construction of the dressings appropriate for the charged particles of the system and, in particular, for the quarks and gluons. Of course, it is almost a truism to insist upon the gauge invariance of all physical quantities in a gauge theory. What is different here is that we are requiring gauge invariance for not just S-matrix elements, but for all the basic constructions in the theory, i.e., also for the propagators and the states of the system. The idea that gauge invariance should dictate how electrons are dressed goes back to Dirac (see Ref. 15and Section 80 of Ref. 16). There are understandable reasons for why this suggestion seems to have gone largely unnoticed. In the context of QED the Bloch-Nordsieck theorem allows one to, at least perturbatively, circumvent the infra-red problems associated with the use of the (gauge non-invariant) Lagrangian fermion. Given that Dirac's expression for the electron is, as we will see, both non-local and non-covariant, it is tempting to dismiss it as a purely formal construction that cannot be accommodated within the standard perturbative, field theoretic catechism. We argue, though, that in QCD such a pragmatic view is less defensible. Indeed, it has long been speculated that the additional infra-red problems found there could lead to a confinement mechanism through some form of infra-red slavery ${ }^{[17-19]}$. However, as this is usually approached within a conventional, local, covariant formulation of quantum field theory, Dirac's non-local, non-covariant, abelian construction has had little impact.

This is not to say, though, that Dirac's arguments have been completely ignored. We have become aware of several groups that have either been directly influenced by Dirac's observations, or have arrived at similar points of view through their own investigations ${ }^{[20,21]}$. In Ref. 22 the obstruction to extending Dirac's treatment of the electron to non-abelian gauge theories has also been noted, and its relation to confinement discussed. Gauge invariance is also seen to take a central role in Ref.'s 23-26 albeit from somewhat different perspectives. We should also, for completeness, mention the work of Kugo and Ojima ${ }^{[27]}$ where confinement is related to gauge invariance (or more properly, BRST invariance), although the connection of that approach to the one presented here is far from clear to us. None of these works, though, deals with the perturbative aspects of the dressing. Hence they are unable to interpret the construction in terms of constituent quarks.

We will now outline the structure of this paper and highlight the key results. After this introduction, in Sect. 2, we summarize the successes of the constituent quark model. This 
short review will serve as a constant source of motivation for the more technical arguments that will follow. Then, in Sect. 3, the basic properties of QCD will be discussed. As well as allowing us to fix our notation, this gives us an opportunity to present several important aspects of gauge theories which will be central to our discussion. In particular, we will discuss the definition of colour charges, proving that the colour charge only has a well defined meaning on gauge invariant states. It immediately follows that the Lagrangian quarks and gluons do not have well defined colour charges. We then recall that charged states are always non-local and that their Lorentz transformations are not the standard ones. Having developed sufficient background material, in Sect. 4 we present explicit dressings of quarks to lowest order in perturbation theory, and show that these dressed quarks have well defined colour charges. This construction makes manifest the non-locality and non-covariance expected from the dressing, and we show that such dressed quarks may be combined to form colourless hadrons in the way commonly done in the constituent quark model. In Sect. 5 we systematise the results of the preceding section, and show how gluons may also be dressed. An algorithm for dressing colour charges is given. The central theme of this section, and one which underlies this paper, is the close relationship between dressings and gauge fixings. This allows us to then identify the existence of the Gribov ambiguity with the breakdown of the non-perturbative dressing of a quark. This unavoidable feature of QCD means that it is impossible to construct asymptotic quark states, and single quarks cannot be observed.

In Sect. 6 we study the practical use of the dressed quark. We see in a one loop calculation that a dressed quark's propagator is gauge invariant and infra-red finite in a mass shell renormalisation scheme where the on-shell quark has the velocity appropriate to its dressing. This allows us to identify Dirac's original dressing as that of a static particle. We further see that it is possible to incorporate quark condensate effects in a gauge invariant fashion into the dressed quark propagator and that they introduce a running mass. In Sect. 7 the interaction between static quarks is discussed. We show that the perturbative interquark static potential may, with the help of the dressings, be obtained in a simpler fashion than is usually the case. We make two proposals for how to extract the confinement scale in such a heavy meson system.

The final part of this paper deals with how light quarks may be dressed. In Sect. 8 the structure of Lorentz transformations in gauge theories is investigated. We see that the Lorentz generators must be modified when they act on charged states. These results are 
then brought to bear on the construction of boosted charges in Sect. 9. Gauge invariant dressings for moving charges are given. In Sect. 10 a summary of our results is presented, along with some speculations on future directions. Finally, there is an appendix where the dressing for a static quark to order $g^{3}$ is explicitly derived. 


\section{The Constituent Quark Model}

The aim of this section is to rapidly review the successes of the constituent quark model. More detailed discussions of this model may be found in Ref.'s 28-30. These results show that, despite confinement, we can still use quarks in low energy hadronic physics although these constituent quarks are not the bare Lagrangian fermions.

Hadrons may be essentially divided into those containing heavy $c$ and $b$ quarks ${ }^{1}$ and those made up of light quarks. Heavy quark-antiquark pairs, quarkonia, have masses which are roughly equal to twice those of the valence quarks. These mesons may be described in terms of non-relativistic potential models which describe confinement in terms of a phenomenological potential. The success of non-relativistic models, and their ability to describe the splitting of the energy levels in terms of a chromomagnetic interaction, is strong evidence that it makes sense to discuss these hadrons in terms of their constituent quarks. The quarks here are dressed quarks but their masses are believed to be close to those of the Lagrangian masses.

It is much more surprising that we can also describe light hadrons in terms of constituent quarks. Recall that the Lagrangian masses of the three light quarks may be estimated to be: $m_{u} \approx 4 \mathrm{MeV}, m_{d} \approx 7 \mathrm{MeV}$ and $m_{s} \approx 150 \mathrm{MeV}$ from chiral symmetry arguments ${ }^{[31]}$. These are more or less negligible compared to the standard hadronic masses. The physical scale which must generate the hadronic masses is the QCD scale, $\Lambda_{\mathrm{QCD}}$, which enters the theory through renormalisation. It turns out, however, to be possible to generate the spectrum of light hadrons remarkably well by assuming they are composed of quark-antiquark pairs or three quarks, which quarks now, however, have larger, so-called, constituent masses. It is, of course, very well known ${ }^{[2,3]}$ that the hadrons can be fitted into multiplets such as the vector octet. A corollary of this is that certain states are forbidden, i.e., they cannot be constructed in the simple quark model out of three spin one-half quarks or from a quark and an antiquark. The problems with the experimental signals for these states is another confirmation of the applicability of the simple (coloured) quark model. At the simplest quantitative level the quark model corresponds to noting that the mass of the rho meson is very roughly equal to two thirds that of the nucleon and the observation that the difference in the masses of a meson formed from two $u$ or $d$ quarks and one constructed out of a strange quark and a single $u$ or $d$ quark is roughly equal to

\footnotetext{
1 The $t$ quark decays too rapidly for hadrons to form.
} 
the mass gap between the latter and a meson solely built out of $s$ quarks. However, much greater accuracy can be attained.

Let us now briefly recall the quantitative precision of the quark model ${ }^{[28,29]}$. The model predicts that the masses of the mesons are given by

$$
m_{\bar{q}^{\prime} q}=m_{\bar{q}^{\prime}}+m_{q}+\Delta_{\text {spin }}^{1}
$$

and those of the baryons should be described by

$$
m_{q q^{\prime} q^{\prime \prime}}=m_{q}+m_{q^{\prime}}+m_{q^{\prime \prime}}+\Delta_{\text {spin }}^{2}
$$

where the $m_{q}$ are the constituent masses of the quarks and the $\Delta_{\text {spin }}^{i}$ describe the hyperfine splitting. This term emanates from a spin-spin interaction produced by one gluon exchange, we shall not go into its exact form here, suffice it to say that it depends upon the masses of the quarks, their spins and the square of the wave function of a quark pair for vanishing separation $^{[29]}$. (For baryons this term entails a sum over all the possible quark pairs.) The model has therefore three free parameters: the mass of the constituent $u$ and $d$ quarks (assumed to be equal), that of the $s$ quark and the square of the wave function.

The following two tables contain the results of fits for mesons and baryons respectively. (The fits are taken from Ref. 29, and experimental values ${ }^{2}$ from Ref. 32.) For the mesons one uses the following constituent quark masses: $m_{u}=m_{d}=310 \mathrm{MeV}$ and $m_{s}=483 \mathrm{MeV}$ and obtains (in units of $\mathrm{MeV}$ ):

$\begin{array}{lrrrrrr}\text { Meson } & \pi & K & \eta & \rho / \omega & K^{*} & \phi \\ \text { Mass (Expt.) } & 137 & 496 & 548 & 769 / 782 & 894 & 1019 \\ \text { Mass (Theory) } & 140 & 485 & 559 & 780 & 896 & 1032\end{array}$

while the baryons may be fitted with slightly different parameters. From the masses $m_{u}=m_{d}=363 \mathrm{MeV}$ and $m_{s}=538 \mathrm{MeV}$ one finds:

$\begin{array}{lrrrrrrrr}\text { Baryon } & N & \Lambda & \Sigma & \Xi & \Delta & \Sigma^{*} & \Xi^{*} & \Omega \\ \text { Mass (Expt.) } & 939 & 1116 & 1193 & 1318 & 1232 & 1385 & 1533 & 1673 \\ \text { Mass (Theory) } & 939 & 1114 & 1179 & 1327 & 1239 & 1381 & 1529 & 1682\end{array}$

The goodness of these fits is remarkable. They show that hadrons may be very well described in terms of dressed, constituent quarks. The constituent masses differ slightly for

2 These experimental values are slightly rounded off and, where necessary, are averages over the various charge states of the hadrons. 
the quarks in mesons and those in baryons. This is not unexpected since they are confined in quite different systems. However, the similarity of the masses is great and supports the view that the dressing of the quarks is primarily responsible for the constituent mass which is only somewhat modified by the dynamics of confinement.

Before looking at further quantitative predictions, we need to recall that the statistics of the constituent quark picture was responsible for the introduction of the colour quantum number ${ }^{[33]}$. The combination of fermionic quarks could only produce a ground state particle like the $\Delta^{++}$(made from three $u$ quarks, with zero angular momentum and parallel spins, i.e., its wave function is completely symmetric) if colour was introduced and one postulated a totally antisymmetric colour wave function, $\epsilon^{a b c}$, for the constituent quarks. Once we introduce colour we may construct general baryonic wave functions with the necessary symmetry properties.

Using then the spin wave functions of three coloured constituent quarks in the various baryons, we can also predict the magnetic moments of the baryons ${ }^{[29]}$. The above baryons all have zero angular momentum and their magnetic moments may all be assumed in the framework of the quark model to be a direct sum of the magnetic moments of the constituent quarks. These last have magnetic moments

$$
\mu_{q}=\frac{e_{q}}{2 m_{q}}
$$

where $e_{q}$ is the electric charge of the quark. One may now, for example, find the ratio of the magnetic moments of the proton and the neutron from their wave functions without reference to the quark masses (assuming only that $m_{u}=m_{d}$ ) and a good agreement is obtained. As far as the absolute values are concerned, one obtains using the constituent quark masses which fitted the masses of the baryons the following predictions (in units of nuclear magnetons):

$\begin{array}{lrrrrrrr}\text { Baryon } & p & n & \Lambda & \Sigma^{+} & \Sigma^{-} & \Xi^{0} & \Xi^{-} \\ \text {Moment (Expt.) } & 2.79 & -1.91 & -0.61 & 2.46 & -1.16 & -1.25 & -0.65 \\ \text { Moment (Theory) } & 2.79 & -1.86 & -0.58 & 2.68 & -1.05 & -1.40 & -0.47\end{array}$

This accuracy is again very satisfactory, especially considering the extraordinary simplicity of the model. We note also that these last predictions test not only the size of the constituent masses but also the symmetry properties of the baryonic wave functions. Further predictions of the quark model may be found elsewhere ${ }^{[28,29]}$. 
The above agreement between experimental data and the predictions of the constituent quark model shows that it is useful to think of hadrons as being made up of dressed quarks; this although the confinement mechanism is conspicuous by its absence in the above considerations. Additionally these are not the current quarks of QCD: the masses are quite different. Furthermore, as we shall see in the following section, the Lagrangian quarks of QCD have ill-defined colour charges because of their gauge dependence, a defect that is at odds with the constituent quark model.

Of course the constituent quark model breaks down if high energy probes are used. This is because such probes test the structure of the constituent quarks and the naive model lacks this structure. The need for structure is seen most simply in the existence of the need for two different limiting masses - the current and constituent masses. The dressing of the constituent (valence) quarks must be made up of gluons and sea quarks. At short distances and very large momenta the coupling is small and the dressing may be to a good approximation neglected. At larger distances we know that the coupling increases and that perturbation theory will collapse, at this stage the quark picture we propose will also break down and we can only talk of hadrons. This should also be reflected in one's choice of effective models.

In the next section we will introduce the QCD concepts required for this paper, in particular we will discuss colour charges and states. In the light of this treatment we will be able to start dressing a Lagrangian quark. 


\section{Colour charges and states in QCD}

In this section we review the structure of QCD as a field theory, paying particular attention to its gauge symmetries and their generators. We will show that colour quantum numbers can only be defined on gauge invariant states. This has direct consequences for any description of coloured fields, i.e., quarks and gluons. Furthermore, we will see that gauge invariant charged states are necessarily both non-local and non-covariant. Hence any approach to constituent quarks necessitates going beyond the traditional, local, covariant formulation of QCD. The implications of this general account of charged states will all be visible in the explicit solutions which we will start to develop in the next section.

As discussed in the introduction, QCD is now widely accepted as the correct field theoretic description of the strong interaction. It is a gauge theory based upon the nonabelian structure group $S U(3)$ rather than the abelian group $U(1)$ familiar from QED. This non-abelian structure manifests itself in the fact that the gauge fields interact not just with the matter fields but also with themselves. The non-linearities inherent in such self interactions obstruct any simple extrapolation from our experience with the structure of QED to QCD.

The basic fields in QCD are the vector fields, $A_{\mu}^{a}$, and the spinor fields $\psi_{n}$. In these the index $a$ ranges over the values $1, \ldots, 8$ (the dimension of the $S U(3)$ group), while $n$ takes three distinct values (the dimension of $S U(3)$ 's fundamental representation). (We will, on occasion, deal with more general gauge theories in which case $S U(3)$ is replaced by an $S U\left(N_{c}\right)$ group.) These fields are conventionally identified with the gluons and the quarks. Although we shall see that this identification is, at best, misleading, it is so ingrained into the physics literature that we will also loosely refer to these fields in this way. When a distinction is important, though, we shall refine this terminology and refer to these fields as the Lagrangian quarks and gluons.

Let $T^{a}$ be a basis of the Lie algebra $s u(3)$. We take these to be anti-hermitian operators in some representation of $S U(3)$ which satisfy the Lie algebra commutator

$$
\left[T^{a}, T^{b}\right]=f_{a b c} T^{c}
$$

where the $f_{a b c}$ are the structure constants of $s u(3)$. For the quark fields the appropriate representation is the fundamental one where we take $T_{m n}^{a}=\left(\lambda^{a} / 2 i\right)_{m n}$, with $\lambda^{a}$ the GellMann matrices. For the gauge fields we use the adjoint representation with $T_{b c}^{a}=-f_{a b c}$. 
The gauge fields can be viewed in a more compact way as a $s u(3)$ valued field $A_{\mu}$, where ${ }^{[34]}$

$$
A_{\mu}:=A_{\mu}^{a} T^{a}
$$

We can recover the components via $A_{\mu}^{a}=-c \operatorname{Tr}\left(A_{\mu} T^{a}\right)$, where $c$ is a constant determined by the representation (in the fundamental representation $c=2$ ).

The field strength is given by

$$
F_{\mu \nu}^{a}=\partial_{\mu} A_{\nu}^{a}-\partial_{\nu} A_{\mu}^{a}+g f_{a b c} A_{\mu}^{b} A_{\nu}^{c}
$$

where $g$ is the coupling constant. The Yang-Mills Lagrangian is then

$$
\mathcal{L}_{\mathrm{YM}}=-\frac{1}{4} F^{\mu \nu a} F_{\mu \nu}^{a}
$$

The covariant derivative acting on the matter fields is

$$
\left(D_{\mu} \psi\right)_{n}=\partial_{\mu} \psi_{n}+g A_{\mu}^{a} T_{n m}^{a} \psi_{m}
$$

and the matter Lagrangian is

$$
\mathcal{L}_{M}=\bar{\psi}\left(i \gamma^{\mu} D_{\mu}-m\right) \psi
$$

The QCD Lagrangian is then the sum of the Yang-Mills and matter ones:

$$
\mathcal{L}_{\mathrm{QCD}}=\mathcal{L}_{\mathrm{YM}}+\mathcal{L}_{\mathrm{M}}
$$

The equations of motion which follow from this are

$$
\left(D_{\mu} F^{\mu \nu}\right)^{a}=g J_{a}^{\nu}
$$

where the covariant derivative is now that appropriate to the adjoint representation, and we have the matter current

$$
J_{a}^{\mu}:=-i \bar{\psi} \gamma^{\mu} T^{a} \psi
$$

In addition to this current we can also define the conserved singlet vector current

$$
J^{\mu}=\bar{\psi} \gamma^{\mu} \psi
$$

In the free quark theory the matter current is also conserved and it may be used to determine the colour charge carried by such quarks. In this way combinations of quarks 
can be constructed with a prescribed colour and, in particular, globally colourless hadrons may be given a non point-like definition. However, when we couple the quarks to gluons in QCD, we see from (3.8) that this matter current is not conserved. As such, its role in characterizing coloured states has been lost. This reflects the fact that gluons also possess colour charges. To find an appropriate conserved colour charge in QCD we first need to review the structure of some of the symmetries of the Lagrangian (3.7).

Among the many symmetries of the QCD Lagrangian, the gauge symmetry is surely primus inter pares. We recall that, by construction, (3.7) is invariant under the transformation

$$
A_{\mu} \rightarrow A_{\mu}^{U}:=U^{-1} A_{\mu} U+\frac{1}{g} U^{-1} \partial_{\mu} U
$$

and

$$
\psi \rightarrow \psi^{U}:=U^{-1} \psi
$$

where $U(x)$ is an element of the group $S U(3)$ (in the appropriate representation) for each point $x$ in spacetime. These gauge transformations contain the rigid transformations where $U(x)=U$ is a constant group element. For such rigid transformations we have

$$
\begin{aligned}
A_{\mu} & \rightarrow U^{-1} A_{\mu} U \\
\psi & \rightarrow U^{-1} \psi
\end{aligned}
$$

The transformations (3.11-3.14) are symmetries of the action, as such they have associated with them conserved Noether currents. Writing $U(x)=e^{\theta(x)}$, where $\theta=\theta^{a} T^{a}$, we find that the Noether current for gauge transformations is

$$
j_{\theta}^{\mu}=-\frac{1}{g} F^{\mu \nu a}\left(D_{\nu} \theta\right)^{a}+J_{a}^{\mu} \theta^{a} .
$$

For the rigid transformations the parameter $\theta^{a}$ is a constant which we can discard to give the conserved current

$$
j_{a}^{\mu}=-f_{a b c} F^{\mu \nu b} A_{\nu}^{c}+J_{a}^{\mu} .
$$

The conservation of this current is a consequence of Noether's theorem and follows directly from (3.8) which can be written as $\partial_{\mu} F^{\mu \nu a}=g j_{a}^{\nu}$.

From (3.15) we expect to be able to identify the generator of gauge transformations with the charge

$$
\begin{aligned}
G(\theta) & =\int d^{3} x j_{\theta}^{0}(x) \\
& =\int d^{3} x G^{a}(x) \theta^{a}(x),
\end{aligned}
$$


where

$$
G^{a}(x)=-\frac{1}{g}\left(D_{i} E_{i}\right)^{a}(x)+J_{a}^{0}(x)
$$

and $E_{i}^{a}(x)=-F_{0 i}^{a}(x)$ is the chromo-electric field.

Using the basic, non-vanishing, equal time (anti)commutators

$$
\left[E_{i}^{a}(x), A_{j}^{b}(y)\right]=i \delta^{a b} \delta_{i j} \delta(x-y)
$$

and

$$
\left[\psi^{\dagger}(x), \psi(y)\right]_{+}=\delta(x-y)
$$

it is straightforward to show that the Noether charge (3.17) generates the transformations

$$
\begin{aligned}
\delta_{\theta} A_{i}^{a}(x) & :=\left[i G(\theta), A_{i}^{a}(x)\right] \\
& =\frac{1}{g}\left(D_{i} \theta\right)^{a}
\end{aligned}
$$

and

$$
\begin{aligned}
\delta_{\theta} \psi(x): & =[i G(\theta), \psi(x)] \\
& =-\theta \psi(x) .
\end{aligned}
$$

These are the infinitesimal forms of the gauge transformations on the $A_{i}$ and $\psi$ fields, but we have not recovered the gauge transformation on the time component $A_{0}$ of the gauge field. The reason for this is that this field is really not a dynamical variable. In the QCD Lagrangian (3.7) it enters as a multiplier field (this is most easily seen from the fact that its momentum $E_{0}^{a}$ can only be of the form $F^{00 a}$, which trivially vanishes). This means that this gauge theory is a constrained system and care must be taken in identifying physical degrees of freedom. Variation of the Lagrangian (3.7) with respect to the multiplier field $A_{0}^{a}$ leads to the further constraints

$$
G^{a}(x)=0,
$$

which are the non-abelian generalizations of Gauss' law.

In the quantum theory we cannot take the constraint (3.23) to hold as an operator equation since this is incompatible with the above commutation relations, rather it is implemented as a restriction on the allowed physical states of the system. So, heuristically, a state $|\psi\rangle$ is said to be physical if

$$
G^{a}(x)|\psi\rangle=0
$$


That this is at least consistent follows from the commutator

$$
\left[G^{a}(x), G^{b}(y)\right]=-i f_{a b c} G^{c}(x) \delta(x-y),
$$

which reflects the fact that Gauss' law is also the generator of the gauge transformations.

On such physical states the expectation values of the constraints are zero: $\left\langle\psi\left|G^{a}\right| \psi\right\rangle=$ 0 . This we can interpret as naturally corresponding to the classical condition (3.23). We note, however, that we may not use this weaker condition to replace (3.24). If we were to use this superficially more appealing property to actually define the physical states then it is simple to see that such a restriction on the expectation values does not pick out a linear subspace of states, i.e., if $\left\langle\psi_{1}\left|G^{a}\right| \psi_{1}\right\rangle=\left\langle\psi_{2}\left|G^{a}\right| \psi_{2}\right\rangle=0$, then $\left(\left\langle\psi_{1}\left|+\left\langle\psi_{2}\right|\right) G^{a}\left(\left|\psi_{1}\right\rangle+\left|\psi_{2}\right\rangle\right)=\right.\right.$ $2 \operatorname{Re}\left\langle\psi_{1}\left|G^{a}\right| \psi_{2}\right\rangle$ which does not necessarily vanish. Hence the weaker condition would imply that there would be no superposition principle.

How are we to interpret the condition (3.24)? This seems to imply that the physical states are gauge invariant, but even in QED this would lead to difficulties. The problem is that the gauge transformations also include the rigid ones where $\theta$ is a constant. In this case the abelian charge becomes the electric charge, and physical states annihilated by this can only have charge zero. This is clearly something of an obstacle to our aim of constructing charged states. To avoid this we must have a non-trivial action of the rigid transformations on the physical states. To determine then what (3.24) means in terms of the gauge invariance of the system we will analyse how the colour charge is to be defined in QCD.

From (3.16) we would expect to identify the colour charge, $Q=Q^{a} T^{a}$, with

$$
\begin{aligned}
Q^{a} & =\int d^{3} x j_{a}^{0}(x) \\
& =\int d^{3} x\left(J_{a}^{0}(x)-f_{a b c} E_{i}^{b}(x) A_{i}^{c}(x)\right) .
\end{aligned}
$$

This is, by construction, conserved but it is not gauge invariant. From our discussion in Sect. 2 we know that constituent quarks have a well-defined colour and the question now naturally arises: how can we reconcile this with the gauge structure of Chromodynamics? The answer hinges on taking the gauge invariance of any physical state seriously. Extending an argument due to Jackiw (see Exercise 2.5 in Ref. 34), we now show that if this charge is acting on a physical state, as defined by (3.24), then we have

$$
j_{a}^{0}=\frac{1}{g} \partial_{i} E_{i}^{a},
$$


and we so find that

$$
\begin{aligned}
Q & =\frac{1}{g} \int d^{3} x \partial_{i} E_{i} \\
& =\frac{1}{g} \lim _{R \rightarrow \infty} \int_{S_{R}^{2}} d \boldsymbol{s} \cdot \boldsymbol{E}
\end{aligned}
$$

where we have used Gauss' theorem to get an integral over the spatial two-sphere $S_{R}^{2}$ of radius $R$. Under a gauge transformation we have $E_{i} \rightarrow U^{-1} E_{i} U$ so that the charge $Q$ becomes $Q^{U}$, where

$$
Q^{U}=\frac{1}{g} \lim _{R \rightarrow \infty} \int_{S_{R}^{2}} d \boldsymbol{s} \cdot U^{-1} \boldsymbol{E} U .
$$

In order to be able to extract the group element from this integral we must assume that it tends to a constant $U_{\infty}$ in a direction independent way. Then

$$
Q^{U}=U_{\infty}^{-1} Q U_{\infty}
$$

and we see that the colour charge acting on physical states is gauge invariant under those gauge transformation that at spatial infinity tend to a constant $U_{\infty}$ which lies in the centre of $\mathrm{SU}(3)$. We postpone for the moment a detailed analysis of the structure of these gauge transformation, and content ourselves with the observation that if $U_{\infty}=1$, the identity element of $S U(3)$, then the colour charge (3.26) is invariant.

We now define the group of local gauge transformations to be those gauge transformations that become the identity at spatial infinity. In addition we have the rigid (global) gauge transformations which are those that are constant in space-time. Note the important distinction between local and rigid gauge transformations: to define colour charge we are forced to restrict local gauge transformations to those which reduce to unity at spatial infinity, this means that the rigid transformations are not a special class of the local ones in this description. We thus see that in QCD we have to consider those gauge transformations which are a combination of such local and rigid transformations.

We know from the instanton structure of QCD, (see also Sect. 5), that the local gauge group is disconnected ${ }^{[34]}$. This means that it is only the part of the group connected to the identity that can be generated from the infinitesimal gauge transformations and hence Gauss' law. Thus, in order for the colour charge to be well defined, the physical states, as defined by (3.24), are invariant under those local gauge transformations that belong to the identity component of the group of all gauge transformations. In a moment we will see that the BRST charge gives a more succinct characterization of these states. 
In summary, we have seen that the conserved colour charge (3.26) is only well defined acting on locally gauge invariant states. Under rigid transformations this charge transforms in the same way as the matter charge constructed out of the matter current (3.9). From its construction, it is clear that we should expect both the quarks and the gluons to carry colour charge. This is in marked contrast to the situation in QED where it is only the electron that has an electric charge, not the photon.

The gauge sector of QCD was initially described by a vector field $A_{\mu}^{a}$ with $4 \times 8$ degrees of freedom (per space time point). We have seen that a kinematical consequence of gauge invariance was that these are reduced to $2 \times 8$ degrees of freedom. (There are $2 \times 8$ constraints coming from the primary constraint $E_{0}^{a}=0$ and Gauss' law.) This is analogous to the reduction in QED from the four-potential to the two transverse polarizations for the photon. This discussion, though, has been at the expense of manifest Lorentz invariance. Now quantum field theory, to a large extent, owes its success to the realization that the ultra-violet structure of the theory is best handled in a manifestly covariant formulation. That is, in a perturbative description, the programme of regularization and then renormalization has only been successfully carried out in local, manifestly covariant theories.

To circumvent the non-covariance inherent in the dynamical content of the QCD Lagrangian (3.7) a Lorentz invariant gauge fixing term may be introduced. A convenient choice is the Lorentz class of gauges

$$
\mathcal{L}_{\xi}=-\frac{1}{2 \xi}\left(\partial_{\mu} A^{\mu a}\right)^{2}
$$

which can be viewed as a generalization of the Lorentz gauge, $\partial_{\mu} A^{\mu a}=0$. The addition of this gauge fixing term has the desired effect of destroying the gauge invariance of the QCD Lagrangian, resulting in all the components of the gauge field being dynamical. This democracy in the dynamics means, though, that we are not allowed to just blithely add such a gauge fixing term to the Lagrangian, since it has evidently changed the physical content of the theory. On top of this, maintaining covariance implies that the state space will have an indefinite metric. In QED, in for example the Feynman gauge (where $\xi=1$ and $\left.\square \partial_{\mu} A^{\mu}=0\right)$, one can project out the unphysical, negative norm states using the Gupta-Bleuler positive frequency condition $\left(\partial_{\mu} A^{\mu}\right)^{+}|\psi\rangle=0$. In QCD, though, this is not possible and additional ghost degrees of freedom must be introduced. Heuristically, as ghosts are fermionic scalars, each one contributes minus one degrees of freedom. So adding 
eight ghost fields $c^{a}$ and eight anti-ghost fields $\bar{c}^{a}$ to the gauged fixed QCD Lagrangian will result in a theory with the expected $4 \times 8-2 \times 8$ degrees of freedom. The actual proof of this claim is far from transparent. The key step in the argument is to note that the ghosts contribute a term

$$
\mathcal{L}_{\text {ghost }}=-\bar{c}^{a} \partial^{\mu} D_{\mu} c^{a}
$$

to the full QCD effective Lagrangian

$$
\mathcal{L}_{\text {eff }}=\mathcal{L}_{\mathrm{QCD}}+\mathcal{L}_{\xi}+\mathcal{L}_{\text {ghost }}
$$

Although gauge invariance has been lost, the action constructed out of this Lagrangian is invariant under BRST transformations. Acting on the gluons and quarks these are:

$$
\begin{aligned}
\delta A_{\mu}^{a} & =\frac{1}{g}\left(D_{\mu} c\right)^{a} \\
\delta \psi & =-c \psi .
\end{aligned}
$$

Comparing these with (3.21) and (3.22), it is clear that there is a close connection between these global (but odd) transformations (3.34) and the original gauge transformations of the system. This simple observation lies at the heart of the success of the BRST method.

This symmetry is generated by a BRST charge $Q_{\mathrm{BRST}}$ which is nilpotent, $Q_{\mathrm{BRST}}^{2}=0$. The physical states are now defined as being BRST invariant; a condition which can be shown ${ }^{[35,36]}$ to recover the states $(3.24)$. The requirement that the BRST invariant states have a well defined colour charge now translates into the condition that the ghosts tend to zero at spatial infinity. So, for example, a ghost field configuration that only depends on time, $c^{a}=c^{a}(t)$, is not allowed. From (3.21), (3.22) and (3.34) we see that for states built out of the gauge and matter fields, BRST invariance is a precise and rather economical way of imposing the condition that these states are invariant under those local gauge transformations that are in the identity component of the group of gauge transformations.

In the rest of this paper we will work almost exclusively with the Lorentz invariant effective QCD Lagrangian (3.33). However, we will also persist, rather perversely, in occasionally describing the physical states as being gauge invariant. This must always be translated into the statement that the states are BRST invariant. Our justification for such an 'economy with the truth' is that there is little intuition in the wider physics community for BRST invariance: gauge invariance is much more appealing physically. As long as one 
is aware of all the caveats needed when applying gauge invariance, this should not lead to any confusion.

The above discussion shows that there exists a local, covariant formulation of QCD. In this description the ultra-violet structure is manageable - renormalization can be proven. What is more, the colour charge is an observable when restricted to BRST invariant states.

Starting from the vacuum, we can build up physical states by applying gauge invariant operators to it. For example, in QCD sum rules the singlet current $J_{\mu}(x)$ may be used to study certain colourless states. To actually construct hadrons, though, we know from our discussion in the introduction that such 'point like' operators are inappropriate. This leaves two options: we can either attach a Wilson line between the two fermions to maintain gauge invariance but at the cost of then solely having charge zero states, or we can look for a gauge invariant generalization of the Lagrangian fermion, $\psi$. It is this second route that we will follow in the rest of this paper: our aim being to show to what extent such charged states can be constructed in QCD, and to analyse their dynamical consequences. That there should be obstructions to fully carrying out this programme is, we will argue, simply a restatement of confinement. The extent to which such coloured charged states can be defined, though, will give a range of validity for the constituent quark model in QCD.

Before embarking on a detailed account of how such charged states are constructed, we wish to end this section with some general remarks on the expected structure of such states. We will argue that, with only a very few assumptions, a picture emerges of the charged sectors that is far removed from the local, covariant stand taken in the standard approaches to quantum field theories. Indeed, many of the unfamiliar techniques that we will be forced to introduce in our later construction of charged states will have natural counterparts in this initial general discussion.

In our description of the colour charge (see the discussion around Eq. 3.28) we assume that such an operator exists and is non-zero on some states. In terms of the field strength, we expect it to fall off like $1 / R^{2}$ at spatial infinity, a fall off rate dictated by finite energy considerations. This allows us to identify the "asymptotic field strength"

$$
F_{\mu \nu}^{\mathrm{as} a}(t, \theta, \phi)=\lim _{R \rightarrow \infty} R^{2} F_{\mu \nu}^{a}(t, R, \theta, \phi) .
$$


We now assume that the physical states are such that the expectation value of the field strength converges to a well defined distribution (for a more precise account of this see, for example, Ref. 37)

$$
f_{\mu \nu}^{a}:=\left\langle\psi\left|F_{\mu \nu}^{\mathrm{as} a}\right| \psi\right\rangle=\lim _{R \rightarrow \infty} R^{2}\left\langle\psi\left|F_{\mu \nu}^{a}\right| \psi\right\rangle
$$

In fact, for what follows, we only need the limit

$$
\Phi^{a}(\boldsymbol{n})=\lim _{R \rightarrow \infty} R^{2}\left\langle\psi\left|\boldsymbol{n} \cdot \boldsymbol{E}^{a}(R \boldsymbol{n})\right| \psi\right\rangle,
$$

which measures the asymptotic chromo-electric flux distribution in the direction $\boldsymbol{n}$.

We note here that we cannot deduce from this alone that the potentials should fall off like $1 / R$ at spatial infinity, since there could be a residual pure gauge term. However, we have seen that in order for the colour charge to be well defined, such a pure gauge must, in fact, be zero.

Given a physical state $|\psi\rangle$, its colour charge from (3.28) and (3.37) is

$$
\left\langle\psi\left|Q^{a}\right| \psi\right\rangle=\int_{S^{2}} d \Omega \Phi^{a}(\boldsymbol{n}) .
$$

That is, as a consequence of Gauss' law, its charge is given by the total chromo-electric flux through the sphere at spatial infinity. In QED we expect to be able to construct charged states, so the abelian version of the limit (3.37) is expected to hold. For QCD isolated coloured charges have not been observed, so the existence of a non-zero limit in (3.37) might be considered questionable. However, to probe the extent to which constituent quarks can be constructed, we now proceed to analyse the consequences of (3.38) in QCD.

States can always be written as $O|0\rangle$, where $O$ is a gauge invariant operator and $|0\rangle$ is the vacuum ${ }^{3}$. In quantum mechanics the (Hermitian) operators correspond to the observables of the system. In gauge theories being Hermitian and gauge invariant is not enough to be able to qualify as an observable: in addition causality dictates that the operator must be local — that is, having support in some compact space-like region ${ }^{[37]}$. In QED the field strength $F_{\mu \nu}$ and current $J^{\mu}$ (both suitably smeared) are observables. In QCD we have the singlet current $J^{\mu}$ and the colour charge $Q^{a}$, for localized sources, as observables. On top of these one can construct observables from localized Wilson loops

3 In QCD we would expect the vacuum to incorporate some non-perturbative structure, so $|0\rangle$ should not be thought of as just the perturbative vacuum. We will return to this point in Sect. 6 . 
and Wilson lines between fermions. Recalling our discussion of the charge operator around equation $(3.29)$, we see that for any such observable $O$ we have ${ }^{[7]}$

$$
\left[Q^{a}, O\right]=\frac{1}{g} \lim _{R \rightarrow \infty} \int_{S_{R}^{2}} d s \cdot\left[\boldsymbol{E}^{a}, O\right]=0
$$

since $O$ has, by definition, compact support and hence vanishes outside of some sphere of finite radius. This implies that any state created from the vacuum by such an observable will have charge zero. Hence charged states are intrinsically non-local.

This argument shows that, even in QED, if one wishes to maintain locality for all fields then charged states cannot exist in nature. Put another way: charged states can only be described by unobservable fields (since they are non-local) ${ }^{[38]}$. A possible response to this is to maintain a zero charge by always including a balance between the charges in the theory (the so called "particle behind the moon" argument of Haag and Kastler ${ }^{[39]}$ ). This is neither very appealing nor is it economical. Indeed, in the context of inflationary cosmology, the charge of our accessible universe is not expected to be zero. This implies that if we want to correctly model the real world then charged states are needed and hence some form of non-locality must be expected. We stress, this does not mean that observables can now be non-local, that would violate causality; rather we must accept into the theory non-local fields that take us from the vacuum to the charged sectors. Although these fields cannot be directly identified with observables due to their non-locality, they will have observable consequences since they create charged states.

We now wish to conclude this section with an account of the surprising impact gauge invariance has on that stalwart symmetry — the Lorentz invariance of the theory. In QED it has been noted ${ }^{[40-43,37]}$ that the well known problems with the infrared structure ${ }^{[10]}$ obstruct the unitary implementation of the Lorentz group: the Lorentz symmetry is said to be spontaneously broken (see, for example, page 281 of Ref. 37). Buchholz's analysis ${ }^{[43]}$ makes it clear that this obstruction is related to the gauge invariance of the system. In Sect. 8 we will present an alternative account and interpretation of this important result that directly uses the gauge invariance of the system. Then, in Sect. 9 we will see how to use these results to construct non-static charged states. For now, though, let us briefly summarize how this result is usually arrived at.

It is a general phenomena in both quantum mechanics and quantum field theory that the state space breaks up into physically distinct sectors - the so called superselection 
sectors of the theory ${ }^{[37]}$. These are closed subspaces of the state space which are invariant under the action of the observables of the theory. In QCD the $\theta$-parameter ${ }^{[34]}$, which labels the $\theta$-vacua, thereby labels different superselection sectors. Another example in QCD are the sectors labelled by triality ${ }^{[44]}$. Both of these types of sectors reflect global properties of the Yang-Mills configuration space: the $\theta$ parameter emerges because the gauge group is disconnected; triality arises because $S U(3)$ has a non-trivial centre. In general we expect many other such sectors arising from the topological structure of the system $^{[45]}$. Furthermore, it is evident that, since it is not possible for a local operator to alter the flux (3.37), the asymptotic field strength also labels different superselection sectors. A consequence of this is that the charge of the system is a label for different superselection sectors ${ }^{[37]}$.

The fact that the asymptotic fields (3.37) label superselection sectors now has a direct consequence for the action of the Lorentz group on the system. Since the asymptotic field of a charged particle can be changed by applying a Lorentz transformation, such a transformation must change the superselection sector. But this contradicts the expectation that the Lorentz generators are observables of the system. This leaves us with two choices: either the Lorentz generators are not gauge invariant, and hence not observables (which is physically unappealing as they will then have no clear action on the true degrees of freedom); or we take their gauge invariance as fundamental and live with the fact that they cannot furnish a representation of the Lorentz group on all the fields (which is, to say the least, an unfamiliar stand in quantum field theory). In Sect. 8 we will show explicitly how these general considerations arise in practice, and how to deal with them. There we will see that on the (local) physical observables and states the Lorentz group does act in a physically acceptable way. However, on the gauge invariant charged states their action changes in quite a dramatic way.

In conclusion, we have seen in this section that gauge theories contain both physical and un-physical degrees of freedom, as well as observable and un-observable gauge invariant operators. Physics dictates much of the mathematical structure associated with the physical observables of the theory, i.e., their locality and Lorentz covariance properties. The conventional (and highly successful) approach to these theories requires that even the un-observable fields share these mathematical structures. We have seen, though, that this utilitarianism fails when charged sectors are allowed and we argue that it is misleading to think of individual quarks or gluons as local, covariant fields. Hence in any approach 
to constituent quarks we must allow for non-locality and the lack of manifest Lorentz invariance. One aim of this paper is to show that one can live with this in quantum field theory. 


\section{Dressing the Quarks}

In the previous section we derived some general results about charged states in gauge theories. In particular we saw that any description of a charged state must be gauge invariant, non-local and non-covariant. This clearly rules out the Lagrangian fermion $\psi$ - which, as we argued earlier, does not correspond to a real field of the sort seen in experiments and has only a very limited asymptotic validity corresponding to the case of the free theory, $g=0$. The aim of this section is to go beyond this and to obtain explicit solutions for charged states in QCD to low orders in perturbation theory. These solutions will then be shown to have well-defined colour charges, which means that they can be combined just as in the naive quark model to form colourless hadrons.

A gauge invariant extension of a fermion may be formed by attaching a string to it. In QED, where similar problems exist with the construction of charged states (although their effects are, as we will see, not so dramatic) such a 'string' electron is:

$$
\psi_{\Gamma}(x) \equiv \exp \left(i e \int_{-\infty}^{\boldsymbol{x}} d \boldsymbol{z}_{i} A_{i}\right) \psi(x)
$$

where the integral runs over some contour, $\Gamma$. (We assume that this contour is local in time; a detailed critique of temporal non-localities in any description of a physical field will be presented in Sect. 5.) By attaching the string to the point at spatial infinity, and using the boundary condition that the gauge transformations reduce to the identity there, the gauge invariance of $\psi_{\Gamma}$ is easily seen. The non-physical aspect of this ansatz is, however, immediately apparent: the contour, $\Gamma$, is arbitrary and the dependence upon it is non-physical. This unnatural dependence may, however, be factorised out. We split the $A_{i}$ fields into the longitudinal and transverse components in the standard way $A_{i} \equiv A_{i}^{T}+A_{i}^{L}$, where $A_{i}^{L} \equiv \frac{\partial_{i} \partial_{j} A_{j}}{\nabla^{2}}$. Recall that the non-local term

$$
\frac{f}{\nabla^{2}}(x) \equiv-\frac{1}{4 \pi} \int d^{3} y \frac{f\left(x_{0}, \boldsymbol{y}\right)}{|\boldsymbol{x}-\boldsymbol{y}|},
$$

is (given our assumptions about the spatial fall-off of the potentials) the inverse Laplacian acting on $f$, i.e., $\nabla^{2} / \nabla^{2}=1$. It is then obvious that (4.1) may be rewritten as

$$
\psi_{\Gamma}(x)=N_{\Gamma} \exp \left(i e \frac{\partial_{i} A_{i}}{\nabla^{2}}(x)\right) \psi(x)
$$


where the dependence on $\Gamma$ is now only present in the first term

$$
N_{\Gamma}(x) \equiv \exp \left(i e \int_{-\infty}^{\boldsymbol{x}} d \boldsymbol{z}_{i} A_{i}^{T}\right) .
$$

Dropping this unphysical first factor, we are forced to use what is left to describe the electron.

In fact we recognise the second term in (4.3) as just the set of fields corresponding to electrons and positrons which was proposed many years ago by Dirac ${ }^{[16]}$. He suggested using

$$
\psi_{\mathrm{c}}(x) \equiv \exp \left(i e \frac{\partial_{i} A_{i}}{\nabla^{2}}(x)\right) \psi(x),
$$

where the $A_{i}(x)$ are the QED gauge fields (which are real) and $e$ is the electromagnetic coupling constant. It is easily seen that these fields fulfill our minimal expectations from Sect. 3, i.e., they are gauge invariant, and (manifestly) non-local and non-covariant. The notation $\psi_{\mathrm{c}}$ is used to signify that this field dresses the charged field $\psi$ with the Coulombic electric field. As such it is describing a static charge in QED. This interpretation can be seen formally as follows: let $|\varepsilon\rangle$ be an eigenstate of the electric field, i.e., we have $E_{i}(x)|\varepsilon\rangle=\varepsilon_{i}(x)|\varepsilon\rangle$. Then consider the (equal time, $\left.x_{0}=y_{0}\right)$ state $\psi_{\mathrm{c}}(y)|\varepsilon\rangle$. It is also an eigenstate of the electric field since,

$$
\begin{aligned}
E_{i}(x) \psi_{\mathrm{c}}(y)|\varepsilon\rangle & =\left[E_{i}(x), \psi_{\mathrm{c}}(y)\right]|\varepsilon\rangle+\psi_{\mathrm{c}}(y) E_{i}(x)|\varepsilon\rangle \\
& =\frac{e}{4 \pi} \frac{\boldsymbol{x}_{i}-\boldsymbol{y}_{i}}{|\boldsymbol{x}-\boldsymbol{y}|^{3}} \psi_{\mathrm{c}}(y)|\varepsilon\rangle+\psi_{\mathrm{c}}(y) \varepsilon_{i}(x)|\varepsilon\rangle \\
& =\left(\varepsilon_{i}(x)+\frac{e}{4 \pi} \frac{\boldsymbol{x}_{i}-\boldsymbol{y}_{i}}{|\boldsymbol{x}-\boldsymbol{y}|^{3}}\right) \psi_{\mathrm{c}}(y)|\varepsilon\rangle .
\end{aligned}
$$

From this we see that introducing $\psi_{\mathrm{c}}(y)$ has changed the electric field by a term corresponding to that from a static charge at the point $\boldsymbol{y}$. This argument is rather appealing, but its reliance on the canonical commutation relations (3.19) implies that it is only really valid for free theories (or the bare fields). For interacting theories an infinite renormalization of the field is needed which could greatly complicate the above argument. In the full theory it is more useful to focus on the Green's functions, and we will see in Sect. 6 that the same interpretation of $\psi_{\mathrm{c}}$ as a static charged field emerges directly from the renormalization of its two-point function.

Note, however, that the string ansatz dismissed above only possesses an electric field along the string contour — which is a further indication of its essentially unphysical nature. We also mention here that Shabanov ${ }^{[46]}$ has shown that if we take a static, non-local 
fermion-antifermion pair joined by a string, so as to ensure gauge invariance, as an initial state and then study its time development, one finds that it radiates and eventually approaches a pair of the Dirac physical fields from (4.5).

We now want to find a description of quarks similar to that of (4.5). We no longer have Dirac's guideline of the Coulombic electric field, but we will let ourselves be led by gauge invariance and the lack of any dependence upon an arbitrary path such as $\Gamma$ above. It is not enough to just replace the coupling $e$ with $g$, and the potential $i A_{i}(x)$ by $A_{i}^{a}(x) T^{a}$ (recall our anti-hermitian conventions for the non-abelian potentials), since gauge invariance is then lost as may be easily seen. However, we note that at lowest order in perturbation theory this direct extension of Dirac's description of the electron to the quark does go through and we can define:

$$
\psi_{\mathrm{c}}^{g^{1}}(x)=\left(1+g \frac{\partial_{i} A_{i}^{a}}{\nabla^{2}}(x) T^{a}\right) \psi(x),
$$

which we may suggestively rewrite as

$$
\psi_{\mathrm{c}}^{g^{1}}(x)=\exp \left(g \frac{\partial_{i} A_{i}^{a}}{\nabla^{2}}(x) T^{a}\right) \psi(x)+O\left(g^{2}\right) .
$$

To see that this is indeed gauge invariant to lowest order in the coupling recall that under a local gauge transformation, $U(x)=\exp \left(g \theta^{a} T^{a}\right)$, we have in perturbation theory:

$$
\psi(x) \rightarrow(1-g \theta(x)) \psi(x)+O\left(g^{2}\right),
$$

and

$$
A_{i}(x) \rightarrow A_{i}(x)+\partial_{i} \theta(x)+O\left(g^{1}\right),
$$

where we note that due to the factor of $1 / g$ in (3.11) it is convenient in such a perturbative expansion in $g$ to include a factor of the coupling in $U$. The factor of $g$ in the exponential of (4.5) also means that we only require the gauge transformation on $A_{i}$ to zeroeth order in $g$. From these we have

$$
\begin{aligned}
\psi_{\mathrm{c}}^{g^{1}}(x) & \rightarrow\left(1+g \frac{\partial_{i} A_{i}^{a}}{\nabla^{2}}(x) T^{a}+g \theta(x)\right)(1-g \theta(x)) \psi(x) \\
& =\left(1+g \frac{\partial_{i} A_{i}^{a}}{\nabla^{2}}(x) T^{a}\right) \psi(x)+O\left(g^{2}\right),
\end{aligned}
$$

which shows the desired invariance. 
Although (4.7) is not gauge invariant at order $g^{2}$ it is possible, with a certain amount of effort, to obtain the correction terms which make it so ${ }^{[8]}$. One finds

$$
\begin{aligned}
\psi_{\mathrm{c}}^{g^{2}}(x)=(1 & +g \frac{\partial_{i} A_{i}^{a}}{\nabla^{2}}(x) T^{a}+\frac{1}{2} g^{2}\left(\frac{\partial_{i} A_{i}^{a}}{\nabla^{2}}(x) T^{a}\right)^{2} \\
& -\frac{1}{2} g^{2} f^{a b c} \frac{1}{\nabla^{2}}\left(A_{j}^{b} \frac{\partial_{j} \partial_{i} A_{i}^{c}}{\nabla^{2}}\right)(x) T^{a} \\
& \left.-\frac{1}{2} g^{2} f^{a b c} \frac{\partial_{j}}{\nabla^{2}}\left(A_{j}^{b} \frac{\partial_{i} A_{i}^{c}}{\nabla^{2}}\right)(x) T^{a}\right) \psi(x) .
\end{aligned}
$$

At this stage we should comment on why we still interpret this field as describing a static quark. Firstly this description reduces in the abelian case $\left(f^{a b c} \rightarrow 0\right)$ to Dirac's description which has the electric field of a static charge. Secondly this is a minimal description: we could imagine adding further separately gauge invariant gluonic field combinations, which would not destroy the gauge invariance of the dressing, but this would unnecessarily raise the energy of the quark.

The gluonic counterpart of this description and a systematic way of obtaining such expressions will be presented in the next section. We now want to see how this quark behaves under the action of the charge operator (3.26) with the eventual aim of seeing how to build colourless objects out of these fields. For simplicity all explicit calculations in the remainder of this section will be in the framework of an $S U(2)$ gauge theory, a more general treatment will be postponed until the next section.

Recall firstly that in the free quark theory (i.e., no gluons) we only have a rigid gauge symmetry and the charge operator reduces to the matter charge

$$
Q_{\text {mat }}^{a}=\int d^{3} x J_{a}^{0}(x)
$$

For the free $S U(2)$ theory we expect the quarks to diagonalize the charge $Q_{\text {mat }}^{3}$ (or more generally the Cartan sub-algebra) where now the antihermitian $T^{a}$ are related to the Pauli matrices by $T^{a}=\sigma^{a} / 2 i$. The addition of a free quark to (say) a charge zero state, $|\Omega\rangle$, alters the eigenvalue of this charge operator by

$$
\begin{aligned}
Q_{\text {mat }}^{3} \psi_{1}(x)|\Omega\rangle & =\frac{i}{2} \psi_{1}(x)|\Omega\rangle \\
Q_{\text {mat }}^{3} \psi_{2}(x)|\Omega\rangle & =-\frac{i}{2} \psi_{2}(x)|\Omega\rangle
\end{aligned}
$$


and a meson with zero net colour charge may be described (as in the constituent quark model) by

$$
\bar{\psi}_{n}(x) \psi_{n}(y)
$$

Under rigid gauge transformations 'red' may become 'blue' etc., but such a meson remains colourless overall, rather as an isospin singlet remains a singlet under isospin rotations, and a 'meson' does not change the charge $Q_{\text {mat }}^{3}$

$$
Q_{\text {mat }}^{3} \bar{\psi}_{n}(x) \Gamma \psi_{n}(y)|\Omega\rangle=0
$$

where $\Gamma$ denotes any potential Lorentz structure. The analogous description of a baryon goes through directly. So much regarding the free quark theory.

In QCD, however, we have a local gauge invariance and it is naively apparent that a configuration of two or more Lagrangian quarks at different points would not stay colourless under local gauge transformations. In fact, as we saw in Sect. 3, we can only associate a charge to gauge invariant fields and although we could introduce, say, strings connecting the fermions, this would be foreign to our philosophy of trying to talk about quarks. Even more significantly perhaps, the charge operator in QCD (unlike in QED) is not the same in the free and the interacting theories, and although we have dressed the quarks above in such a way that they are perturbatively gauge invariant, we have dressed them with gluons which themselves are coloured objects. It is therefore not self-evident that we can straightforwardly combine the dressed quarks in the usual way to form hadrons.

To see that this is in fact possible consider the lowest order description of an $S U(2)$ dressed quark from (4.7). The commutator of the full charge with the dressed quark receives two terms (one from the gluons and one from the fermions) and, although it is not immediately obvious, they can be shown by explicit calculation to combine to give

$$
\begin{aligned}
Q^{3} \psi_{\mathrm{c} 1}^{g^{1}}(x)|\Omega\rangle & =\frac{i}{2} \psi_{\mathrm{c} 1}^{g^{1}}(x)|\Omega\rangle \\
Q^{3} \psi_{\mathrm{c} 2}{ }^{g^{1}}(x)|\Omega\rangle & =-\frac{i}{2} \psi_{\mathrm{c} 2}^{g^{1}}(x)|\Omega\rangle .
\end{aligned}
$$

This is just what we obtained in the free (glue-less) theory. We so learn that the physical, dressed quark fields have a well defined colour charge which has the properties of the charge of the fermion in the free theory. This means that we can indeed combine the dressed quarks so as to form, e.g., (perturbatively) colourless, mesonic objects:

$$
\bar{\psi}_{n}(x) \psi_{n}(y) \rightarrow \bar{\psi}_{\mathrm{c} n}^{g^{1}}(x) \psi_{\mathrm{c} n}^{g^{1}}(y)
$$


and analogous baryonic structures. Recalling the distinction we were earlier forced to make between local and rigid gauge transformations (see just after Eq. 3.30), we see that this explicit calculation may be understood rather simply: although the dressed quarks are invariant under local gauge transformations, they transform just like the Lagrangian quark under rigid transformations. The colour charge is, however, the charge associated with the Noether current for such rigid transformations and so we would indeed expect that perturbatively dressed quarks have colour charges like those of the bare ones in the free theory. This property will be also be visible in all of the extensions (higher orders in $g$, non-static) of the dressed quark (4.12) which will be presented below.

This is a very satisfying result which explains one of the more surprising parts of the constituent quark model: it means that we can combine coloured dressed quarks to form mesons and baryons just as we would naively do in the model. This, for example, allows us to write the wave function of a baryon as a product of a colour wave function for the constituent, dressed quarks with the remaining wave function (i.e., the spatial, spin and flavour parts) in accord with the Pauli principle. This aspect of hadronic spectroscopy was of course the initial motivation for the introduction of the colour quantum number. A further implication of this result is that we have not yet seen colour screening. The colour charges will be screened by renormalisation effects and by interactions between the quarks when we bring them together in bound states. We will present our initial studies of the interactions between such quarks in Sect. 7 .

Before we can, however, start to combine the dressed quarks to form hadrons, we are now confronted by several immediate questions. Firstly, can we systematise the derivation of these dressed quark fields and are we able to dress quarks (and gluons) non-perturbatively? Secondly, these fields are both non-local and non-covariant: can one actually work with them in a quantum field theory such as QCD? In particular, can we carry though a programme of regularisation and renormalisation for these fields and their Green's functions? We will address these important issues (in the above order) in the following two sections. 


\section{The Breakdown of the Quark Description}

In this section we explain how the construction of the perturbative quarks can be generalized and extended both to all orders in perturbation theory and to the gluons. The key step in the argument is the recognition of the intimate link between gauge fixing and dressing any charged object. As well as providing a simple way of generating the perturbative dressing, and showing that the colour charges of dressed quarks are those of the constituent quark model to any order in perturbation theory, this link will let us prove that in QCD there is an obstruction to extending these results beyond perturbation theory. This lack of any gauge invariant description of a single quark or gluon underlies colour confinement.

We have seen that it is possible to construct perturbatively physical quarks, at least to low orders in the coupling. The solution (4.12) had the characteristic non-locality and noncovariance expected of such a charged object. A natural question to ask is what expression is (4.12) the low order approximation to? In addition to this, for completeness, we would like to see a similar description of the gluons.

In order to dress the quark $\psi$ and make it gauge invariant we need a gauge field dependent element of $S U(3)$ that transforms under the gauge transformation (3.11) in the same way as $\psi$, i.e., we need to find $h \in S U(3)$ such that under local gauge transformations we have

$$
h \rightarrow h^{U}=U^{-1} h .
$$

Then a physical quark can be identified with the BRST invariant combination

$$
\psi_{\text {phys }}=h^{-1} \psi
$$

From (4.12) we see that our expression for the static quark corresponds to taking $\psi_{\mathrm{c}}^{g^{2}}=$ $h_{\mathrm{c}}^{-1} \psi$ with $h_{\mathrm{c}}=e^{-v_{\mathrm{c}}}+O\left(g^{3}\right)$ and, to this order,

$$
v_{\mathrm{c}}=g \frac{\partial_{i} A_{i}}{\nabla^{2}}-\frac{1}{2} g^{2} \frac{1}{\nabla^{2}}\left[A_{j}, \frac{\partial_{j} \partial_{i} A_{i}}{\nabla^{2}}\right]-\frac{1}{2} g^{2} \frac{\partial_{j}}{\nabla^{2}}\left[A_{j}, \frac{\partial_{i} A_{i}}{\nabla^{2}}\right] .
$$

In this expression we have reverted to the compact expression (3.2) for Lie-algebra valued fields and hence the commutators are Lie-algebra commutators. We note that since $\partial_{i}$ and $1 / \nabla^{2}$ commute, this expression can also be written as a total divergence (see the appendix):

$$
v_{\mathrm{c}}=\frac{\partial_{j}}{\nabla^{2}}\left(g A_{j}+g^{2}\left[\frac{\partial_{i} A_{i}}{\nabla^{2}}, A_{j}\right]+\frac{1}{2} g^{2}\left[\frac{\partial_{j} \partial_{i} A_{i}}{\nabla^{2}}, \frac{\partial_{k} A_{k}}{\nabla^{2}}\right]\right)
$$


Given a dressing $h$ satisfying (5.1) then the BRST invariant gluonic field is identified with the transformed field $A_{i}^{h}$, i.e., with

$$
\left(A_{\mathrm{phys}}\right)_{i}:=h^{-1} A_{i} h+\frac{1}{g} h^{-1} \partial_{i} h .
$$

To see that this is also gauge invariant note that under the gauge transformation $A \rightarrow A^{U}$ we have

$$
\begin{aligned}
\left(A_{\mathrm{phys}}\right)_{i} & \rightarrow\left(h^{U}\right)^{-1} A_{i}^{U} h^{U}+\frac{1}{g}\left(h^{U}\right)^{-1} \partial_{i} h^{U} \\
& =h^{-1} U A_{i}^{U} U^{-1} h+\frac{1}{g} h^{-1}\left(U \partial_{i} U^{-1}\right) h+\frac{1}{g} h^{-1} \partial_{i} h \\
& =h^{-1}\left(A_{i}+\frac{1}{g} \partial_{i} U U^{-1}\right) h-\frac{1}{g} h^{-1}\left(\partial_{i} U U^{-1}\right) h+\frac{1}{g} h^{-1} \partial_{i} h=\left(A_{\text {phys }}\right)_{i} .
\end{aligned}
$$

Substituting expression (5.3) into (5.5) yields the physical gluonic field $\left(A_{\mathrm{c}}\right)_{i}:=A_{i}^{h_{\mathrm{c}}}$ which is to $O\left(g^{1}\right)$

$$
\left(A_{\mathrm{c}}^{g^{1}}\right)_{i}=\left(\delta_{i j}-\frac{\partial_{i} \partial_{j}}{\nabla^{2}}\right)\left(A_{j}+g\left[\frac{\partial_{k} A_{k}}{\nabla^{2}}, A_{j}\right]+\frac{1}{2} g\left[\frac{\partial_{j} \partial_{k} A_{k}}{\nabla^{2}}, \frac{\partial_{l} A_{l}}{\nabla^{2}}\right]\right)
$$

We have thus reduced the problem of finding gauge invariant, dressed quarks and gluons to that of constructing such a field $h$. In other words, once we have found $h$ we can directly construct dressed quarks and gluons which may then be combined to form hadronic bound states. Different $h$ dressing functions will be seen to correspond to both static and nonstatic quarks.

Having seen the utility of being able to construct the field $h$ transforming as in (5.1), we now want to demonstrate that the existence of such a dressing field is equivalent to finding a gauge fixing condition. This relation between gauge fixing and the dressings will allow us to both study the non-perturbative aspects of quarks and gluons and will give us a highly efficient algorithm for extending the perturbative results of the last section. That there is a connection between dressing and gauge fixing should not come as too much of a surprise. Indeed, from the specific static example (5.3) we see that, at least to this low order in $g$, the Coulomb gauge $\partial_{i} A_{i}=0$ removes the dressing $\left(v_{\mathrm{c}}=0\right)$. In order to show that this is true to all orders for the static quark, and that an analogous result holds for any dressing, we need to first define what is meant by a gauge fixing condition in this context. 
We have argued that the QCD effective Lagrangian (3.33) must contain a gauge fixing term. Recall that such a term is generically a function of the potentials, $\chi^{a}(A)$, where the Fadeev-Popov determinant, $\operatorname{det}\left|\delta \chi\left(A^{U}\right) / \delta U^{\prime}\right|$, does not vanish ${ }^{[48]}$. This gauge fixing is generally taken to be covariant in order to simplify the ultra-violet structure of the theory. Dressing the quark will involve a further gauge fixing which is not this Lagrangian gauge fixing, and we will henceforth refer to it as the dressing gauge fixing. What we will see is that the dressing gauge is the gauge choice that removes the explicit dressing from the quarks, resulting in the identification of the Lagrangian fermion with the physical quark field.

In QED we see from (4.5) that in the Coulomb gauge $\psi_{\mathrm{c}}=\psi$, and hence then the Lagrangian fermion $\psi$ is a physical charged field. This appears to be at odds with our general result that such fields are intrinsically non-local. We recall, though, that in the Coulomb gauge, which is clearly non-covariant, the vector fields have space-time commutator (see p. 484 of Ref. 10)

$$
\left[A_{i}(x), A_{j}(y)\right]=i\left(\delta_{i j}-\frac{\partial_{i} \partial_{j}}{\nabla^{2}}\right) \triangle(x-y),
$$

where $\triangle(x)$ is, for the free field, the Jordan-Pauli function $\triangle(x)=-\frac{1}{2 \pi} \varepsilon\left(x_{0}\right) \delta\left(x^{2}\right)$. Locality would imply that this commutator vanishes for space like separations. But the term

$$
\begin{aligned}
\frac{1}{\nabla^{2}} \triangle(x) & =\frac{1}{8 \pi|\underline{x}|}(|t+| \underline{x}||-|t-| \underline{x}||) \\
& =\frac{1}{4 \pi} \varepsilon(t) \theta\left(x^{2}\right)+\frac{t}{4 \pi|\underline{x}|} \theta\left(-x^{2}\right)
\end{aligned}
$$

clearly does not vanish for $x^{2}<0$. In this sense, the non-locality in the charged sector is no longer manifest in this gauge - but it is definitely there! We note in this example that we are still free to impose conditions on the temporal component of the potential. So the radiation gauge would also remove the dressing in this case (recall that this is the Coulomb gauge and Ampère's law combined and hence involves two gauge fixing conditions). The implementation of this type of gauge fixing in the path integral formalism is, though, quite involved $^{[47]}$.

Although the Coulomb gauge dressing will play a central role in this paper, it is not the only dressing available to us. Indeed in Sect. 9 a whole class of dressings will be presented describing boosted quarks. Before we get involved in analysing precisely how gauge fixing and dressings go together, though, we need to address a rather natural question: is it 
possible to find a dressing that is removed in a covariant gauge? Clearly, in QED, we can formally construct the gauge invariant and apparently covariant field

$$
\psi_{\mathrm{cov}}(x)=\exp \left(i g \frac{\partial_{\mu} A^{\mu}}{\square}\right) \psi(x) .
$$

Now the Lorentz gauge $\partial_{\mu} A^{\mu}=0$ is both covariant and local. So in this gauge the dressing described in (5.10) would vanish and we have a local covariant charged field $\psi$ ! Now this really is at odds with our general discussion, and there is a temptation to keep (5.10) and conclude that the general results were just idle mathematical speculation! A less impulsive response, though, is needed here. Both (4.5) and (5.10) are no more than symbolic expressions - we must give an, if not rigorous then at least operational, prescription for how they are to be defined. In the context of perturbative field theory we want to construct the propagator for these fields which will involve their time ordered products: i.e., $\left\langle 0\left|T \bar{\psi}_{\text {cov }}(x) \psi_{\text {cov }}(y)\right| 0\right\rangle$. Now (4.5) is only spatially non-local and, as we shall see in great detail in the next section, its propagator can be constructed using reasonably standard field theory techniques. However, the covariant expression (5.10) is, additionally, non-local in time. Again this might seem to present no real obstacle as the overall time ordering should sort out the (admittedly complicated) ordering in the fields. But we must remember that these fields are interacting fields — we are in the Heisenberg picture. In order to develop a perturbative description we need something akin to the Gell-Mann and Low description of the propagator in terms of time ordered products of free fields. To do this we have to assume that in the distant past and future these Heisenberg fields (and their vacuum) "agree" with the free fields ${ }^{[37]}$. But given that these fields are nonlocal in time such an identification is impossible, and hence a perturbative description of (5.10) is not possible. These problems can be avoided in the Euclidean regime if we only deal with the Wightman functions associated with the field (5.10). The only physical interpretation of the construction is then that these correspond to two-point functions for the high temperature regime for some five dimensional gauge theory. Hence the fields (5.10) cannot be given a meaning in a four dimensional gauge theory and, we argue, do not correspond to any physical dressing of the charged particles. The conclusion we reach from this discussion is that the dressing gauge fixing is never covariant, i.e., the nonlocality is only in the spatial directions.

It is useful here to restate this important distinction between the gauge fixings: the Lagrangian gauge fixing that we employ will be chosen to be covariant so that standard 
perturbative techniques can be used; the dressing gauge fixing will, in contrast, necessarily be non-covariant so that charged sectors can be constructed.

We now want to make one further restriction on the dressing before starting our general analysis. In contrast to the discussion above, this new restriction is not essential to our formalism, but will significantly simplify the presentation. The problem that we want to side-step is that in the effective Lagrangian (3.33) the generators for the gauge transformations on the gauge fields split into the Gauss law part (3.18) and the primary constaint, $E_{0}$, which is the momentum conjugate to $A_{0}$. We have seen that Gauss' law generates the local gauge transformations on the spatial components of the gauge field. Being simply a momentum, though, the primary constraint only generates a translation of the temporal components, and as such it does not appear to generate the expected gauge transformations on $A_{0}$. However, we are neglecting the ghost fields in this, and in a more careful analysis ${ }^{[36]}$ one finds that the momentum conjugate to the anti-ghosts smears the primary constraint. When the momentum variables are then eliminated the expected BRST transformation on the temporal component of the gauge field is recovered. To avoid this level of complexity, though, we will make the further restriction that the dressing field be constructed only out of the spatial components of the gauge field. This we will see covers all the dynamical configurations of the dressing needed for this paper (i.e., all boosted states for a quark). The extension to a more general dressing is an interesting topic, but one that we will not concern ourselves with in this paper.

Given these restrictions on the dressing, it is now quite easy to show that a dressing field transforming as in (5.1) implies the existence of a gauge fixing condition. Recall from our discussion in Sect. 3 that it is Gauss' law that generates the gauge transformations. Hence $h^{U}=e^{i G(\theta)} h e^{-i G(\theta)}$, where $U=e^{\theta}$. Thus, to lowest order in $\theta(x)=\theta^{a}(x) T^{a}$, we have from $(5.1)$

$$
i\left[G_{a}(x), h(y)\right]=-T^{a} h(x) \delta(x-y) .
$$

Writing $h(y)=e^{-v(y)}$, with $v(y)=v_{b}(y) T^{b}$, this gives $i\left[G_{a}(x), v_{b}(y)\right]=\delta_{a b} \delta(x-y)+$ terms which vanish when $v$ is set equal to zero. That is, using the idea of weak equivalence familiar from constrained dynamics ${ }^{[36]}$, we have that the infinitesimal version of (5.1) is the (weak) equation

$$
i\left[G_{a}(x), v_{b}(y)\right] \approx \delta_{a b} \delta(x-y) .
$$

The matrix $i\left[G_{a}(x), v_{b}(y)\right]$ is precisely the Fadeev-Popov matrix one would construct for functions $v_{a}$ if we were viewing them as gauge fixing conditions ${ }^{[48]}$. Indeed, the condition 
(5.12) tells us that the Fadeev-Popov determinant associated with this gauge fixing is non-zero, and hence it is a valid gauge fixing condition.

Our aim now is twofold: firstly we want to show that the converse to this holds, i.e., that a gauge fixing condition $\chi^{a}(A)=0$ can be used to construct the dressing $h$. Secondly we will demonstrate that (5.12) does not have a general solution in a non-abelian gauge theory, i.e., there is no complete nonperturbative description of an asymptotic quark or gluon field and we should not expect to see such fields. Before starting the general discussion, let us see how the first point is realised in QED and the Coulomb gauge. As we have said before, the electromagnetic potential $A$ should really be thought of as belonging to an orbit of gauge related potentials which, in this abelian case, are described by $\mathcal{O}_{A}=\left\{A_{i}+\partial_{i} \Lambda\right\}$, where $\Lambda$ is a scalar. The Coulomb gauge condition picks out a unique representative from each such orbit. Thus, for any $A \in \mathcal{O}_{A}$ there must be a potential dependent scalar $v$ such that $\partial_{i}\left(A_{i}+\partial_{i} v\right)=0$. Clearly we have $v=-\partial_{i} A_{i} / \nabla^{2}$. By construction, for another gauge related potential $A_{i}+\partial_{i} \Lambda$ the scalar $v$ transforms to $v-\Lambda$. Thus the gauge invariant (Coulomb gauge satisfying) potential is $A_{i}^{h}=A_{i}-\frac{i}{e} h^{-1} \partial_{i} h$, with $h=e^{i e v}$. Having constructed the dressing field $h$ from the Coulomb gauge fixing condition on the potentials, we can directly write down the (static) electron using the general formula (5.2).

We now want to extend this argument both to more general gauge fixing conditions and to non-abelian gauge theories. As well as providing an efficient algorithm for perturbatively constructing the dressing (see the appendix) this discussion will also show why this construction cannot be extended in the non-abelian theory to a non-perturbative dressing. However, since what follows is of a slightly higher mathematical sophistication than the rest of this work, let us summarise how gauge fixing is used in the construction of the dressing field $h$.

The strategy in the general situation is the same as in the simple example above: we use gauge fixing in the Yang-Mills sector to construct $h$, then use (5.2) to dress the quark. In the Yang-Mills configuration space we denote the orbit of the gauge group that goes through the potential $A$ by

$$
\mathcal{O}_{A}:=\left\{A^{U}: U \in \mathcal{G}\right\}
$$

where $\mathcal{G}$ is the group of all gauge transformations. Again, a gauge fixing condition $\chi=0$ will 'slice' the orbits, picking a representative from each one. Given a potential $A$, we now define $h$ to be the ( $A$-dependent) element of $\mathcal{G}$ that takes the potential $A$ to the point in 


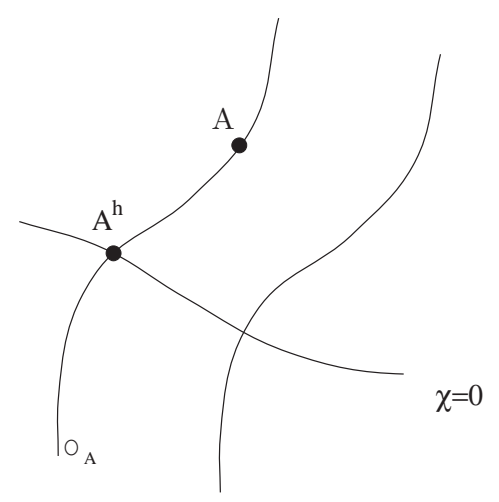

Figure 5.1 A diagram showing how gauge fixing is used to define the dressing.

the orbit $\mathcal{O}_{A}$ where the gauge fixing condition holds, i.e., so that $\chi\left(A^{h}\right)=0$. Pictorially this construction is summarized in Fig. 5.1.

To show that this construction gives a dressing with the correct transformation properties, and that the Gribov ambiguity implies that it can only be defined perturbatively, we need to briefly review some of the more geometric aspects of gauge theories and gauge fixing. We will conclude this section with a brief discussion of the physical implications of this result.

Some care is needed in the precise identification of the Yang-Mills configuration space and we can only hope here to give an honest and, hopefully, intuitive account of the rich structures to be found there. For more details we refer to Ref.'s 49-51. The first thing that we need to do is worry a little about the asymptotic form of the fields. Finiteness of the energy implies that the gauge fields must at least tend to a pure gauge at spatial infinity. However, we have seen that in order to construct a colour charge, any gauge transformation that is not rigid must tend to the identity at spatial infinity. This allows us to identify the Yang-Mills configuration space $\mathcal{A}$ with the set of all potentials: $\mathcal{A}=\left\{A_{\mu}^{a}(x)\right\}$ that tend to zero at least as fast as $1 / R$. One can show that this can be made into an infinite dimensional smooth manifold (modelled now on a Hilbert space). We denote the group of local gauge transformations by $\mathcal{G}$, and this can also be viewed as an infinite dimensional Lie group. The upshot of this is that the spaces of interest in Yang-Mills theory are analytically quite complicated due to the fact that they are infinite dimensional, but their qualititive structures (such as their topology) have much in common with more familiar spaces such as Lie groups and, as we shall see, coset spaces.

The group $\mathcal{G}$ has a (right) action on the space $\mathcal{A}$ given by $A_{\mu} \rightarrow A_{\mu}^{U}$, where $U \in \mathcal{G}$. 
We call this a right action since $\left(A^{U_{1}}\right)^{U_{2}}=A^{U_{1} U_{2}}$. This $\mathcal{G}$ action is essentially free ${ }^{4}$ so the orbit of $\mathcal{G}$ through a point $A \in \mathcal{A}$ is a copy of the group $\mathcal{G}$, i.e., being free implies that $\mathcal{O}_{A} \approx \mathcal{G}$.

The physical Yang-Mills configuration space is identified with the space of orbits $\mathcal{A} / \mathcal{G}$, this is again a smooth infinite dimensional manifold. The mapping $\pi: \mathcal{A} \rightarrow \mathcal{A} / \mathcal{G}$, given by $\pi(A)=\mathcal{O}_{A}$ is a projection onto the physical configuration space. A gauge fixing condition allows us to construct a mapping (cross-section) going the other way, i.e., gives us a mapping $\sigma: \mathcal{A} / \mathcal{G} \rightarrow \mathcal{A}$, which will let us view the physical configurations as special elements of the extended configuration space $\mathcal{A}$. This in its turn will allow us to disentangle the true degrees of freedom from the others in $\mathcal{A}$

To see this let us assume that $\chi^{a}(A)$ is a smooth gauge fixing condition: the nonvanishing of the Faddeev-Popov determinant then implies that the surface $\chi^{a}=0$ intersects each orbit once. This allows us to define a cross-section $\sigma$ by

$$
\sigma\left(\mathcal{O}_{A}\right)=\{\text { the unique element in the orbit where the gauge fixing holds }\}
$$

We can now use this cross-section to separate out the true dynamics from the gauge directions in $\mathcal{A}$. Consider the mapping $i$ from the product space $\mathcal{A} / \mathcal{G} \times \mathcal{G}$ to $\mathcal{A}$ given by

$$
i\left(\mathcal{O}_{A}, U\right)=\sigma\left(\mathcal{O}_{A}\right)^{U}
$$

that is, take the point in the orbit where the gauge fixing holds and then apply the gauge transformation $U$ to it. Since the $\mathcal{G}$ action on $\mathcal{A}$ is assumed to be free, this mapping is both one-to-one and onto.

On the product space the gauge action takes a very simple form

$$
\left(\mathcal{O}_{A}, U_{1}\right) \rightarrow\left(\mathcal{O}_{A}, U_{1}\right)^{U_{2}}:=\left(\mathcal{O}_{A}, U_{1} U_{2}\right)
$$

which allows us to clearly isolate the gauge invariant, and hence physical, structures. The mapping $i$ makes clear the role of gauge fixing in isolating the true degrees of freedom, i.e., to make manifest the physical content of gauge theories we exploit gauge fixing to go

4 This is one of the places where things can get a little involved. As we understand it ${ }^{[51]}$, those connections which have a non-trivial little group in $\mathcal{G}$ are deemed reducible. By lumping gauge fields together with the same little group the Yang-Mills configuration space breaks up into different strata. On each such strata, though, the spaces are much the same. Hence this complication does not alter the main conclusions we wish to reach about gauge fixing. 
from the product space $\mathcal{A} / \mathcal{G} \times \mathcal{G}$, where the physical content is manifest to the jumbledup space $\mathcal{A}$, where there is a highly non-trivial mixing of the physical and un-physical degrees of freedom. Of course, we really want to go the other way around and for this we need the inverse mapping to $i$. This is easy enough to construct, define the mapping $j: \mathcal{A} \rightarrow \mathcal{A} / \mathcal{G} \times \mathcal{G}$ by

$$
j(A)=\left(\pi(A), h^{-1}(A)\right),
$$

where $h^{-1}(A)$ is here defined to be that unique element of $\mathcal{G}$ that takes $\sigma\left(\mathcal{O}_{A}\right)$, the gauge fixed element of the orbit through $A$, back to $A$. That is,

$$
\chi^{a}\left(A^{h}\right)=0 .
$$

That $j$ is the inverse to the mapping $i$ follows easily from the definitions. For example we have

$$
\begin{aligned}
i \circ j(A) & =i\left(\pi(A), h^{-1}(A)\right) \\
& =\left(A^{h}\right)^{h^{-1}} \\
& =A .
\end{aligned}
$$

Now if we apply a gauge transformation to both sides of (5.17) we find that $j\left(A^{U}\right)=$ $\left(\pi\left(A^{U}\right), h^{-1}\left(A^{U}\right)\right)$ and $\left(\pi(A), h^{-1}(A)\right)^{U}=\left(\pi(A), h^{-1}(A) U\right)$, using (5.16). Thus we must have $h^{-1}\left(A^{U}\right)=h^{-1}(A) U$, and we have recovered the dressing transformation (5.1).

In summary, we have seen that in order to build a dressing field for the quarks we can use a gauge fixing condition in the gauge sector to construct $h$. The dressing of the quark is then given by (5.2). That this process leads to an efficient algorithm for finding the dressing field to higher order in $g$ is demonstrated in the appendix where the dressing for the static quark to order $g^{3}$ is derived from the Coulomb (dressing) gauge condition.

As an immediate application of this description of how the dressing field is constructed we can now give an elementary proof of the important result that the dressed quarks transform as irreducible representations of $\mathrm{SU}\left(N_{c}\right)$ under rigid transformations. (Recall that in (4.17) we showed this for $N_{c}=2$ only to order $g$.)

We are required to show that under the rigid transformation (3.13) the dressing transforms as $h \rightarrow \tilde{h}=U^{-1} h U$, so that the dressed quark (5.2) transforms in the same way as the free quarks; $\psi_{\text {phys }} \rightarrow \tilde{\psi}_{\text {phys }}=U^{-1} \psi_{\text {phys }}$; under rigid gauge transformations (recall the distinction between local and rigid gauge transformations made after Eq. 3.30). We know that $A^{h}$ is a potential, so it transforms under a rigid transformation as $A^{h} \rightarrow \widetilde{A^{h}}=U^{-1} A^{h} U$. 
We now need to see that $\widetilde{A^{h}}$ is in the same orbit as $\tilde{A}=U^{-1} A U$ : this may be restated as there being a field dependent $\tilde{h} \in \mathcal{G}$ such that $\tilde{A}^{\tilde{h}}=\widetilde{A^{h}}$. It is now simple to show that $\tilde{h}=U^{-1} h U$ has the required properties (recall that $U$ is rigid).

The final topic we want to address in this section is the obstruction to extending this construction of the dressing beyond perturbation theory. From our discussion we have seen that a dressing for the quark is equivalent to finding a gauge fixing. So the existence of a dressing is inextricably connected with the existence of a gauge fixing condition. Now Gribov $^{[52]}$ has explicitly demonstrated that the Coulomb gauge in Yang-Mills theory does not exist outside of perturbation theory. What he showed was that the condition

$$
\partial_{i}\left(A_{i}^{h}\right)=0
$$

does not uniquely fix $h$. Hence there is not a unique element in the gauge orbit $\mathcal{O}_{A}$ satisfying the Coulomb gauge condition and thus the section (5.14) cannot be constructed. This is not an artifact of the Coulomb gauge condition and, as shown by Singer ${ }^{[49]}$, occurs for all gauge fixing conditions defined on $\mathcal{A}$. This has become known as the Gribov ambiguity. This existence of this ambiguity is central to our account of confinement in QCD, hence it is helpful to briefly explain how it arises.

We have identified the group of all local gauge transformations $\mathcal{G}$ with the mappings from space time $\mathbb{R}^{4}$ to the structure group $S U(3)$ which tend to the constant 1 at spatial infinity. Our restriction to dressings only made out of the spatial components of the gauge fields implies that we are not interested in the time dependence of these local gauge transformation, thus we can view the gauge group $\mathcal{G}$ as mapping from space $\mathbb{R}^{3}$ to $S U(3)$ which tend to 1 at infinity. This gauge group has lots of interesting topological structures that it inherits from the group $S U(3)$. This follows from the fact that the homotopy groups of $\mathcal{G}$ are simply related to those of $S U(3)$ by the relation

$$
\pi_{n}(\mathcal{G})=\pi_{n+3}(S U(3))
$$

The homotopy groups of $S U(3)$ are well known ${ }^{[53]}$, and we note that $\pi_{3}(S U(3))=\mathbb{Z}$ and $\pi_{5}(S U(3))=\mathbb{Z}$. The first of these implies that

$$
\pi_{0}(\mathcal{G})=\mathbb{Z}
$$

To understand what this means we recall that the zeroth homotopy group measures the number of disconnected components a space has. Thus (5.21) implies that the gauge 
group is disconnected, having an infinite number of components. This is responsible for the instanton structure of QCD. One can see this disconnectedness explicitly by noting that the function on $\mathcal{G}$ given by

$$
\omega(U)=\frac{1}{24 \pi^{2}} \int d^{3} x \epsilon^{i j k} \operatorname{Tr}\left(U^{-1} \partial_{i} U U^{-1} \partial_{j} U U^{-1} \partial_{k} U\right)
$$

is invariant under the right action $U \rightarrow U e^{\theta}$, i.e., it is invariant under gauge transformations in the identity component of $\mathcal{G}$. In particular we see that on the identity component $\omega\left(e^{\theta}\right)=0$. However, it is possible to find gauge group elements $U$ such that $\omega(U)$ is not zero ${ }^{[34]}$, so these elements cannot be in the identity component of $\mathcal{G}$ and $\mathcal{G}$ is thus disconnected.

So how does this topology in $\mathcal{G}$ relate to gauge fixing? Well, if $\mathcal{G}$ is disconnected then so is the product space $V \times \mathcal{G}$ for any space $V$. In particular $\mathcal{A} / \mathcal{G} \times \mathcal{G}$ must be disconnected. If a gauge fixing exists then we have seen that $\mathcal{A} / \mathcal{G} \times \mathcal{G}$ is diffeomorphic to the Yang-Mills configuration space $\mathcal{A}$. Hence $\mathcal{A}$ must be disconnected. But this is not true, for if $A_{1}$ and $A_{2}$ are any elements of $\mathcal{A}$ then

$$
A(t):=t A_{2}+(1-t) A_{1}
$$

for $t \in[0,1]$, is a line of Yang-Mills potentials in $\mathcal{A}$ from $A_{1}$ to $A_{2}$ - the space $\mathcal{A}$ is thus connected! From this contradiction we must conclude that the spaces $\mathcal{A} / G \times \mathcal{G}$ and $\mathcal{A}$ are not diffeomorphic and hence there can be no gauge fixing.

Now it might be argued that the fact that the group $\mathcal{G}$ is disconnected really means that we should be just investigating the reduction from $\mathcal{A}$ to the coset space of $\mathcal{A}$ with the identity component of $\mathcal{G}$. Indeed the $\theta$-parameter in QCD measures the extent to which the states are invariant under the full group $\mathcal{G}$ and only for $\theta=0$ are they fully invariant. However, even the identity component of $\mathcal{G}$ has a rich topological structure that obstructs the dressing gauge fixing in much the same way as this $\pi_{0}$ result. Indeed the fact that $\pi_{5}(S U(3))=\mathbb{Z}$ implies that

$$
\pi_{2}(\mathcal{G})=\mathbb{Z}
$$

This means that there are non-contractable two-spheres in $\mathcal{G}$, and the existence of a perfect gauge fixing would imply the same for $\mathcal{A}$. This again is not the case since (5.22) implies that $\mathcal{A}$ is topologically trivial. 
In conclusion, we have seen in this section that it is possible to construct dressed quarks and gluons, at least perturbatively, and that such a dressing is closely related to a non-covariant gauge fixing condition. As a consequence of this, we have seen that a nonperturbative dressing would amount to the existence of a globally well defined gauge fixing condition, which is not possible due to the Gribov ambiguity. We have sketched a proof of this deep result - one of the very few rigorously known facts about the non-perturbative structure of QCD. Given that physical quarks would need to be dressed, this shows that it is not possible to construct a non-perturbative asymptotic quark field. This, we propose, is a direct proof of quark confinement. We recall, though, that the experimental results from deep-inelastic scattering are understood in terms of freely moving quarks inside the nucleon and that the naive quark model also shows that hadrons may be described to a good extent in terms of constituent quarks. In other words, although free quarks are not experimentally seen, many aspect of hadronic physics can be described in terms of one or other sort of quark. We now want to address the important question of whether the perturbatively dressed quarks developed here can be used to describe constituent quarks and the fields seen in deep-inelastic scattering. The field theoretic side of this identification will be the topic of the next section. 


\section{The Dynamics of Dressed Quarks}

We now want to see how far we can get using dressed quark fields in practical calculations. We stress again that the standard approach to quantum field theory makes much use of locality and covariance in order to push through the renormalisation programme. Since the dressed quarks (4.12) and gluons (5.7) are neither local nor covariant we will in this section investigate the practicability of working with these fields by performing one-loop studies of the quark propagator both in perturbation theory and in the framework of the operator product expansion (OPE). To retain as many as possible of the advantages of covariance in such calculations, and also to demonstrate the gauge invariant nature of the results, we will always work in a general Lorentz gauge. We will see that the one-loop perturbative propagator of the static quark can be renormalised on-shell in a gauge invariant manner without any trace of an infra-red divergence. The second half of this section shows how the QCD vacuum structure can be incorporated through the OPE and that this yields a gauge invariant, running mass for the dressed quarks.

\section{i) Perturbation Theory of the Constituent Quark Propagator}

Before we proceed to the details of the calculation we wish to first recall some details of the usual one-loop propagator (i.e., diagram Fig. 6.1a) in the Lorentz gauges.

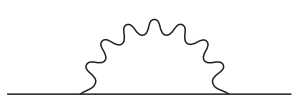

a

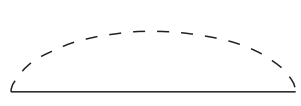

$\mathrm{b}$

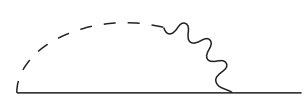

C

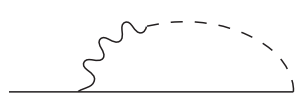

d

Figure 6.1 The one loop contributions to the physical propagator. Solid lines correspond to the fermion propagator, wavy ones to the vector boson propagator and dashed lines represent the projection of the vector boson propagator that comes from the dressing function.

This diagram is, up to a factor, the same in both QED and QCD. Its contribution to the propagator has a logarithmic ultra-violet divergence. This can be dealt with in the standard way (see, for example, Ref. 54). Most important for our purposes is the fact that if one attempts to perform a mass-shell renormalisation one finds an infra-red divergence. This means that we cannot straightforwardly choose a subtraction point such that the external fermions are on shell, i.e., this latter divergence is telling us that we are not able 
to write a renormalised propagator which represents a free on-shell fermion. It is wellknown $^{[11,10]}$ that the Coulomb force of QED falls off so slowly at infinity that we cannot ignore it and use free fermions as our asymptotic fields and this is the origin of the infra-red divergence. Various people have considered redividing the QED Hamiltonian into a new interaction term and a term which would lead to different asymptotic fields ${ }^{[12,55]}$. Essentially the problem may also be understood as a consequence of the photon being massless. An infinite number of soft photons may and must be added to, say, an electron state and one is thus naturally led to using coherent states to describe charged particles ${ }^{[56-58]}$. The photon propagator does not suffer from such divergences since it is chargeless and does not directly interact with the Coulombic fields produced by (and associated with) any charged particles.

In QCD we do not have a description of the long distance nature of the force between bare charges since quarks and gluons are confined. This is supposed to be due to an increase of the strong force at larger distances and so it would seem only logical that we also cannot employ asymptotic Lagrangian quark or gluon fields in QCD. Indeed the QCD Green's functions display infra-red divergences worse ${ }^{[59]}$ than those of QED and it has previously been suggested that they are at the root of confinement ${ }^{[17-19]}$.

The standard methods ${ }^{[10,54,59]}$ of circumventing the infra-red divergence are to give the photon (gluon) a tiny mass or to not perform a mass shell renormalisation scheme. This last option implying that we do not having an asymptotically on-shell particle. What we want to see below is will the dressing of the fermion suggested above remove the infra-red divergence and permit us to put it asymptotically on-shell?

Before starting the detailed perturbative calculation, it is worth recalling that it is known $^{[41,43]}$ that these infra-red divergences are a manifestation of the fact that the mass operator does not have a discrete set of normalizable eigenstates in the charged sector of the theory. As such, the infra-particle ideas of Schroer ${ }^{[60]}$ are usually used to discuss these states. Our approach is in marked contrast to this. The states created by the dressed fields are non-normalizable states and it is through this process that infra-red finite results can emerge. In fact the states created have much in common with coherent states, and it would be interesting to see in a more precise way how dressing the charged states relates to the standard ${ }^{5}$ use of coherent states in the infra-red sector.

5 We also note that the good infra-red properties of the Fried-Yennie gauge ${ }^{[61]}$ can be understood through the gaugeon formalism (see, for example, page 125 of Ref. 35). It is not clear, though, how this interesting construction relates to dressing the charged fields due to its covariant nature. 
Finally we further recall that it is known that the quark pole mass is gauge parameter independent, which may be shown to be a consequence of generalised Ward (Nielsen) identities ${ }^{[63]}$, and that the $Z_{2}$ fermion wave function renormalisation constant in an on-shell scheme has also been shown to be gauge invariant up to two loops by explicit calculation ${ }^{[62]}$. Thus armed we can move on to the field-theoretic description of the dressed propagator.

The propagator of the static dressed quark (4.12) receives at one loop contributions from the four diagrams of Fig. 6.1. These correspond respectively to the usual interaction vertices of the free fermion propagator (6.1a), gluonic corrections which emerge purely from the dressing $(6.1 \mathrm{~b})$ and corrections which receive contributions from both the dressing and the normal interaction vertices $(6.1 \mathrm{c} \& \mathrm{~d})$. Note that the dressing projects out certain components of the full vector boson propagator. Since we wish to preserve BRST invariance we will always use dimensional regularisation which means that tadpole diagrams, where we take both of the $g^{2}$ corrections from one of the dressing functions alone, vanish. Note also that diagrams such as Fig. 6.1b need to be considered at the level of the propagator.

Rather than calculate the diagrams one at a time we choose to first combine them all at integrand level. Note that since the diagrams are, up to colour factors, the same for QED and QCD we drop colour indices henceforth. The first step is to check if the propagator of the gauge invariant dressed fermion is itself gauge invariant. Each of the four diagrams in Fig. 6.1 has a piece linear in the gauge parameter, $\xi$. A little algebra shows that their sum reduces to tadpoles which we recall vanish in dimensional regularisation. Any possible gauge dependence thus cancels ${ }^{[14]}$.

Working in Feynman gauge one now attaches where necessary external factors of $(\not p-m)(\not p-m)^{-1}$ in such a way that a free propagator is present left and right of each of the terms from the four diagrams. This allows us to better combine them. Dropping some tadpoles one finds after a certain amount of algebra that the integral may be reduced to that which we would have from the usual fermion propagator (Fig. 6.1a) evaluated in Coulomb gauge, i.e., we have

$$
S^{\prime}(p)=g^{2} S_{0}(p) \int \frac{d^{2 \omega} k}{(2 \pi)^{2 \omega}} \gamma_{\mu} S_{0}(p-k) \gamma_{\nu} D_{\mathrm{c}}^{\mu \nu}(k) S_{0}(p),
$$

where $D_{\mathrm{c}}^{\mu \nu}$ is the vector boson propagator in Coulomb gauge. This is of course what we would expect - the exponential factors in the dressing reduce to unity in Coulomb gauge $\left(\partial_{i} A_{i}=0\right)$. 
A reasonable amount is known about perturbation theory for electrodynamics in Coulomb gauge ${ }^{[64-67]}$. We will most closely follow Ref. 67, although our notation will deviate from his in several places. The regulated propagator

$$
S^{\prime}(p)=\frac{1}{\not p-m-\Sigma(p)},
$$

is related to the renormalised propagator, $S_{\mathrm{R}}(p)$, by

$$
S^{\prime}(p)=Z_{2} S_{\mathrm{R}}(p)
$$

Writing the inverse regularised propagator as

$$
\not p-m-\Sigma(p)=a \not p+b m+c \not p_{0},
$$

where $\not p_{0}=p_{0} \gamma_{0}$ and $a, b$ and $c$ are as yet unknown functions depending upon $p^{2}$ and $p_{0}^{2}$, we see that

$$
S^{\prime}(p)=\frac{a p+c \not p_{0}-m b}{m^{2} \Delta^{2}}
$$

where

$$
\Delta^{2}\left(p^{2}, p_{0}^{2}\right)=a^{2} p^{2}+c^{2} p_{0}^{2}+2 a c p_{0}^{2}-b^{2} m^{2} .
$$

The physical mass is determined by

$$
\Delta^{2}\left(m^{2}, p_{0}\right)=0
$$

This determines the mass renormalisation. We stress again that this is known to be gauge parameter independent in the class of covariant gauges.

Writing the mass as a sum of the bare mass and the mass shift, $m=m_{0}+\delta m$, one finds the following forms for the three functions in (6.4) to one loop in $2 \omega=(1,2 \omega-1)$ dimensions ${ }^{[67]}$

$$
\begin{aligned}
& a\left(p^{2}, p_{0}\right)=1+\frac{\alpha}{4 \pi \hat{\epsilon}}+\frac{\alpha}{4 \pi}\left(\frac{19}{6}-\int_{0}^{1} \frac{d x}{\sqrt{x}}(1-x) \log \mathcal{X}-2 \int_{0}^{1} d x(1-x) \log \mathcal{Y}\right. \\
&\left.+2 \int_{0}^{1} d x \sqrt{x} \int_{0}^{1} d u \log \mathcal{Z}\right), \\
& b\left(p^{2}, p_{0}\right)=-1-\frac{\alpha}{\pi}\left(\frac{1}{\hat{\epsilon}}+1\right)+\frac{\delta m}{m}+\frac{\alpha}{4 \pi}\left(\int_{0}^{1} \frac{d x}{\sqrt{x}} \log \mathcal{X}+2 \int_{0}^{1} d x \log \mathcal{Y}\right), \\
& c\left(p^{2}, p_{0}\right)=\frac{\alpha}{4 \pi}\left(-\frac{8}{3}+\int_{0}^{1} \frac{d x}{\sqrt{x}}(1-x) \log \mathcal{X}-2 \int_{0}^{1} d x \sqrt{x} \int_{0}^{1} d u \log \mathcal{Z}\right),
\end{aligned}
$$


where

$$
\frac{1}{\hat{\epsilon}}=\frac{1}{2-\omega}-\gamma_{E}+\log (4 \pi)
$$

and

$$
\begin{aligned}
& \mathcal{X}=1+\frac{\boldsymbol{p}^{2}}{m^{2}}(1-x), \\
& \mathcal{Y}=1-\frac{p^{2}}{m^{2}}(1-x), \\
& \mathcal{Z}=1-\frac{p_{0}^{2}}{m^{2}}(1-u)+\frac{\boldsymbol{p}^{2}}{m^{2}}(1-x u)-i \epsilon .
\end{aligned}
$$

The work of Ref. 67 shows that the reality properties of the full propagator (it aquires an imaginary part for $p^{2}>m^{2}$ ) make physical sense. Note further that although some noncovariant integrals must be performed to obtain these results, the integrals are well-defined and the situation is therefore in marked contrast to axial gauges where an integration prescription needs to be introduced ${ }^{[68,47]}$.

Armed with these formulae we can study the mass shift, $\delta m$. Since it is gauge invariant we would not expect it to depend upon $p_{0}$ and indeed explicit calculation shows that

$$
\frac{\partial \delta m}{\partial p_{0}}=0
$$

which was assumed in Ref. 67. The calculation of $\delta m$ is most easily performed for $p_{0}=m$, i.e., the static mass shell since there we find that (6.7) simplifies to ${ }^{6}$

$$
\tilde{a}+\tilde{b}+\tilde{c}=0
$$

where $\tilde{a}$ denotes that the function is taken at its static mass shell (i.e., $\tilde{a}=a\left(p^{2}=m^{2}, p_{0}=\right.$ $m$ ), etc.). Since this is linear in $\alpha$ it may be straightforwardly solved while at other (nonstatic) mass shell momenta this is not the case and we must expand in the coupling only retaining terms up to order $\alpha$. One finds ${ }^{[67]}$

$$
\delta m=\frac{\alpha m}{4 \pi}\left(\frac{3}{\hat{\epsilon}}+4\right),
$$

which is the covariant gauge result. This demonstrates that the gauge invariant mass of the usual fermion propagator is just the mass of the dressed fermion.

To fully renormalise the dressed fermion propagator we now consider $Z_{2}$. We first consider the static mass shell. In other words we choose our renormalisation point to be

\footnotetext{
6 A factor of $\frac{\xi^{2}}{m^{2}}$ in Eq. 34 of Ref. 67 is superfluous.
} 
at $p=(m, 0,0,0)$, which we stress is what our earlier considerations, i.e., the fact that the electric field associated to the dressed fermion is that of a static charge, would suggest. The renormalisation condition is then that as $p \rightarrow(m, 0,0,0)$ we have

$$
S_{\mathrm{R}}(p) \rightarrow \frac{m \gamma_{0}+m}{p^{2}-m^{2}}
$$

which corresponds to a static asymptotic field. (This renormalisation condition was used in Ref. 67, the motivation there was that this was a reasonable renormalisation point for an electron in positronium where one might expect the momenta to be small. Below we will see that this choice is in fact forced upon us by the theory.)

The full propagator in this limit is

$$
S^{\prime}(p) \rightarrow \frac{m \tilde{a} \gamma_{0}+m \tilde{c} \gamma_{0}-m \tilde{b}}{\left.m^{2}\left(p^{2}-m^{2}\right)\left[\partial / \partial p^{2} \Delta\left(p^{2}, p_{0}\right)\right]\right|_{p^{2}=m^{2}, p_{0}=m}}
$$

which from (6.11) may be recast in the form

$$
S^{\prime}(p) \rightarrow \frac{\tilde{b}\left(m \gamma_{0}+m\right)}{\left(p^{2}-m^{2}\right)\left[2 m^{2} \tilde{b}\left(\tilde{a}^{\prime}+\tilde{b}^{\prime}+\tilde{c}^{\prime}\right)-\tilde{a}^{2}\right]}
$$

where the obvious notation $\tilde{a}^{\prime}=\partial /\left.\partial p^{2} a\left(p^{2}, p_{0}\right)\right|_{p^{2}=m^{2}, p_{0}=m}$ applies. Comparing (6.14) and (6.12) we see that the multiplicative renormalisation condition (6.3) holds. Substituting one finds that

$$
Z_{2}=1-\frac{\alpha}{4 \pi} \frac{1}{\hat{\epsilon}}
$$

This is a very attractive result. We see that $Z_{2}$ is not plagued by an infra-red divergence - in contrast to the covariant gauge propagator. This implies that one can use this field as an asymptotic one (at least in the static two-point function). This is strong confirmation that the dressing we use is physical even at the quantum level.

This mass shell renormalisation of the dressed fermion propagator at one loop is now complete. We have seen that the mass shift is gauge invariant and equal to that of the usual fermion in covariant gauges. The wave function renormalisation constant, $Z_{2}$, is, however, very different to that in covariant gauges - it is infra-red finite and could be multiplicatively renormalised. (This last is in contrast to axial gauges, where multiplicative renormalisation is often impossible ${ }^{[68]}$.) However, we wish to now mention a further difference between the renormalisation of the physical fermion and the usual 
covariant approach. In the renormalised propagator of, say, $\phi^{4}$ theory we find after a mass shell renormalisation that the renormalised propagator approaches a free field propagator if the particle is put on shell; since the propagator only depends upon $p^{2}$ it makes no difference in which way it is put on shell. The renormalised propagator which we have obtained above depends upon both $p^{2}$ and $p_{0}^{2}$ and only looks like a free propagator on the static mass shell. The interpretation that the propagator describes a free field on shell is not open to us for other, non-static, mass shell momenta. This is a reflection of the breakdown of the Lorentz boost symmetry and we wish to now study it further.

We have argued that the dressing of the fermion is correct for a static charge. Let us consider what happens if we use a non-static mass shell condition. To be explicit we consider the moving mass shell point $p=m(5 / 4,0,0,3 / 4)=m \eta^{*}$, i.e., $\eta^{*}$ is a (not purely temporal) unit vector. We have already seen above that this will have the same mass shift, $\delta m$, and this may also be explicitly verified from the condition here. Explicitly, $\delta m$ may be found from

$$
\tilde{a}_{1}+\tilde{b}_{1}+\frac{25}{16} \tilde{c}_{1}=0
$$

where the subscripts denote that only terms of order $\alpha$ in $a, b$ and $c$ are to be retained (e.g., $\left.a=1+\alpha a_{1}+\cdots\right)$ and the tildes now of course refer to evaluating the functions at the moving pole. The usual result emerges from (6.16). A problem, however, rears its head when we attempt to evaluate $Z_{2}$. The on-shell renormalisation condition (6.12) now reads

$$
S_{\mathrm{R}}(p) \rightarrow \frac{\frac{5}{4} m \gamma_{0}-\frac{3}{4} m \gamma_{3}+m}{p^{2}-m^{2}}=\frac{m \eta^{*}+m}{p^{2}-m^{2}}, \quad \text { as } \quad p \rightarrow m \eta^{*}
$$

Compare this with the full propagator $S^{\prime}(p)$ which near the moving pole is

$$
S^{\prime}(p) \rightarrow \frac{\frac{5}{4} m(\tilde{a}+\tilde{c}) \gamma_{0}-\frac{3}{4} m \tilde{a} \gamma_{3}-\tilde{b} m}{\left(p^{2}-m^{2}\right) m^{2}\left[\partial /\left.\partial p^{2} \Delta\right|_{\left.p^{2}=m^{2}, p_{0}=\frac{25}{16} m^{2}\right]}\right.},
$$

where the derivative in the denominator is evaluated at the non-static pole position. To first order in $\alpha$ this may be rewritten as the sum of two terms

$$
\begin{aligned}
S^{\prime}(p) \rightarrow & \frac{m \eta^{*}+m}{\left(p^{2}-m^{2}\right) m^{2}\left[\partial /\left.\partial p^{2} \Delta\right|_{\left.p^{2}=m^{2}, p_{0}=\frac{25}{16} m^{2}\right]}\right]} \\
& +\alpha \frac{\frac{5}{4} m\left(\tilde{a}_{1}+\tilde{c}_{1}\right) \gamma_{0}-\frac{3}{4} m \tilde{a}_{1} \gamma_{3}-\tilde{b}_{1} m}{p^{2}-m^{2}}
\end{aligned}
$$

We remark that $\Delta^{2}$ is to be expanded in the coupling. 
It is apparent from the second term in this last equation that the renormalisation is not now multiplicative and explicit calculations bear this out. The non-multiplicative term may be calculated and is seen to be non-vanishing but both ultra-violet and infra-red finite. This is in accord with the work of Heckathorn ${ }^{[66]}$ who showed that the UV poles were multiplicatively renormalisable.

Consider the first term on the RHS of (6.18). It is visible that the UV poles are as in the static mass shell scheme. A short calculation shows, however, that there is now a new infra-red divergence of the form

$$
\int_{0}^{1} d x \frac{\sqrt{x}}{25-9 x} \int_{0}^{1} \frac{d u}{u} .
$$

The interpretation of this divergence is clear. We have calculated the one-loop, dressed propagator corresponding to a static charge. If we use a static renormalisation point we can perform a multiplicative, infra-red finite, mass shell renormalisation. If we choose any non-static mass shell renormalisation point, even for small velocities, we acquire not only non-multiplicative terms but also an infra-red problem since the dressing we employ is only suitable for a static charge. This is an explicit manifestation of the non-covariant nature of the dressing, which we claimed in Sect. 3 and we will return to this in Sect.'s 8 and 9.

The results of this subsection show the great sensitivity of the theory to the type of dressing employed. In Sect. 9 we will study how to boost a charge and how the dressing must then be altered. We will in particular see that Coulomb gauge does not then remove the dressing, which indicates why it is not possible to carry out a non-static mass shell renormalisation scheme for static charges. We conclude this subsection by repeating that no problems were encountered in renormalising the physical fields as long as the correct renormalisation scheme was used.

\section{ii) Operator Product Expansion of the Constituent Quark Propagator}

Notwithstanding the success of the above perturbative description of the dressed quarks, it is natural to want more. Low energy hadronic physics cannot be understood solely in terms of perturbation theory and, although we may hope to describe constituent quarks in hadrons as (at short distances) weakly interacting quasi-particles, it seems likely that the dressed quarks ought to receive some non-perturbative input which should, e.g., generate a rather larger mass than the current one. The rest of this section will concern the interaction of the perturbatively dressed quarks with the non-perturbative QCD vacuum. 
A widely applied method of incorporating some non-perturbative QCD physics is the operator product expansion (OPE) as used in QCD sum rules. The OPE ${ }^{[69]}$ for the product of two local operators takes the general form of a weak equality valid between states:

$$
A(x) B(0) \approx \Sigma_{n} C_{n} \mathcal{O}_{n}, \quad \text { as } x_{\mu} \rightarrow 0,
$$

and it is used to separate short distance (the $C_{n}$ ) and long distance effects (the $\mathcal{O}_{n}$ ). The former coefficients may be calculated perturbatively while the latter are non-perturbative operators which, at the current level of our understanding of QCD, are best estimated from phenomenology.

In the form of QCD sum rules ${ }^{[70]}$ this has developed into a very productive way of predicting hadron masses and investigating other hadronic properties. In the sum rules the short distance effects are the perturbatively calculable coefficients of various universal, non-perturbative condensates in correlation functions of certain gauge-invariant currents. These are then compared with the results of models (resonance saturation) for the same correlation functions and the model parameters (e.g., the mass of the relevant hadron) may be obtained from a fit. The condensates (whose values are themselves obtained from fits) are the vacuum expectation values of certain operators. They are essentially used to parameterise the non-perturbative nature of the QCD vacuum and are to be thought of as unknown functions of the QCD scale rather than new parameters. A simple example is the quark condensate, $\langle\bar{\psi} \psi\rangle$, whose value may be extracted from the PCAC relation, $\left(m_{u}+m_{d}\right)\langle\bar{\psi} \psi\rangle=-m_{\pi}^{2} f_{\pi}^{2}$, which relates the light quark masses, the pion form factor and the pion mass to a condensate whose value is a measure of the extent of spontaneous chiral symmetry breaking. The OPE corrections generally fall off as powers of the momenta flowing through the Green's function or correlation function under consideration. So for large momenta they are negligible, while for very small momenta the OPE breaks down but in some middle region they are hoped to be both important and reliably calculable.

We now want to see how the leading order effects of the non-perturbative vacuum influence the propagator of the dressed quark. It is not obvious that the OPE can be directly applied to the non-local dressed fields. However, the central fermion fields are local and so we may hope that at least the coefficient of the quark condensate may be found. Specifically we want to check that this lowest order term from the OPE yields a gauge-invariant correction to the propagator and to discover its exact form. 


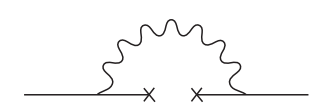

a

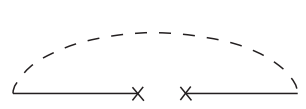

$\mathrm{b}$

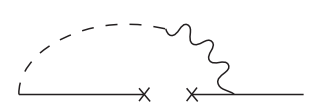

C

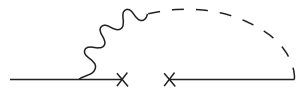

d

Figure 6.2 Insertion of quark condensates into the dressed propagator. The crosses denote quark vacuum insertions. Other notation is as in Fig. 6.1.

We recall in this connection that Politzer suggested ${ }^{[71]}$ many years ago that the OPE of the Lagrangian quark propagator, and in particular the contribution from the quark condensate, $\langle\bar{\psi} \psi\rangle$, could generate the constituent quark mass. It has later been realised that his result was highly gauge dependent. What we will see, however, is that this idea finds a natural application in the dressed quarks and that it there leads to a gaugeindependent, non-perturbative correction to the physical quark propagator. We now begin by summarizing the OPE of the usual propagator ${ }^{7}$.

The first non-perturbative term in the OPE of the usual quark propagator comes from the quark condensate, $\langle\bar{\psi} \psi\rangle$. It arises from Fig. 6.2a. Politzer worked at lowest order in the quark mass and obtained ${ }^{8}$ in Landau gauge the following contribution to the quark self energy

$$
\Sigma\left(p^{2}\right)=\frac{3\left(N_{c}^{2}-1\right) \pi \alpha_{s}\langle\bar{\psi} \psi\rangle}{2 N_{c}^{2} p^{2}} .
$$

It was then argued that this could be understood as an effective, running mass and such ideas have enjoyed some popularity in phenomenology. The mass falls off rapidly at high momenta and the mass scale introduced by (6.20) for initially chiral quarks is (using $\left.\langle\bar{\psi} \psi\rangle=(-270 \mathrm{MeV})^{3}\right)$ of a size which it is natural to want to identify with the constituent quark mass of the light $u$ and $d$ quarks. Unfortunately a more exact analysis shows, as we now discuss, that this mass term in the standard propagator does not in fact lend itself to any straightforward interpretation.

Generally in $D$ dimensions for an $S U\left(N_{c}\right)$ gauge group and to first order in the quark mass one finds ${ }^{[73]}$ in an arbitrary Lorentz gauge from Fig. 6.2a:

$$
\Sigma\left(p^{2}\right)=\frac{\left(N_{c}^{2}-1\right) \pi \alpha_{s}\langle m \bar{\psi} \psi\rangle}{2 N_{c}^{2} p^{2}}\left[\frac{2 \xi(2-D)}{D} \frac{\not p}{p^{2}}+(D-1+\xi) \frac{1}{m}\right]
$$

7 There are different ways of calculating the OPE corrections. Ref.72 provides a recent discussion.

8 A factor was corrected in Ref.73. 
This shows that this correction to the propagator is gauge dependent and hence cannot be associated with any physical meaning.

In fact the OPE of a gauge dependent quantity such as this propagator is even more subtle than this (for a review see Ref. 74). Although $\langle\bar{\psi} \psi\rangle$ is the only gauge and Lorentz invariant dimension three condensate, there are several such quark (and gluon) condensates of dimension four and (6.21) is actually too simple. To obtain (6.21) use was made of the equation of motion for the quark condensate, $\langle\bar{\psi}(\not D-m) \psi\rangle=0$. In QCD sum rules, where one works with correlation functions of gauge (BRST) invariant currents this is valid since it is known ${ }^{[75]}$ that the only operators which can occur there are gauge invariant ones (such as the $\langle\bar{\psi} \psi\rangle$ or $\left\langle G^{2}\right\rangle$ condensates), equation of motion operators such as the above (which can be set to zero in physical states) and BRST variations (whose expectation values vanish). This means that we are entitled in the sum rules to use the equation of motion to replace $\langle\bar{\psi} \not \partial \psi\rangle$ by $\langle m \bar{\psi} \psi\rangle$ since the $\langle\bar{\psi} g A \mathcal{\psi}\rangle$ terms will take care of themselves. (For an explicit demonstration of this in the OPE of the two-point correlation function of the singlet vector current see Ref. 76.) Since the quark propagator is not gauge invariant this use of the equation of motion is not so useful. This fundamental difference between the QCD propagators and the gauge invariant sum rules means that the condensates do not have to appear in particular combinations in the propagators and so it is necessary to calculate the coefficients of all the condensates of a particular dimension. The OPE of the propagator at first order in the quark mass and leading order in $\alpha_{s}$ is found ${ }^{[76]}$, from the diagrams of Fig.'s 6.2a and other additional diagrams, to be such that the condensates enter the OPE of the propagator in gauge dependent combinations and with gauge dependent coefficients. No direct physical meaning may be read off from this OPE of the usual propagator, although these results may be used to constrain solutions of the SchwingerDyson equations ${ }^{[77]}$. In what follows we will restrict ourselves to considering the dimension three condensate, $\langle\bar{\psi} \psi\rangle$.

We have seen above that the pole mass of the usual propagator is gauge invariant in perturbation theory and that this is understood theoretically. It has been argued that the OPE correction to the the pole mass is also gauge invariant ${ }^{[78]}$. This may, e.g., be seen from (6.21) by setting the mass to the pole mass and it follows that at this pole the explicit gauge parameter dependence cancels. We point out, however, that, since the quark condensate is negative, the pole mass which would follow from (6.21) would be negative. (For Euclidean momenta the effective mass is, although gauge dependent, 
indeed positive.) These sign problems notwithstanding, we do not see a gauge independent pole if all dimension four quark condensates are included ${ }^{[76]}$. It may be that summing higher condensates and repeatedly using the equation of motion to replace $\left\langle\bar{\psi}(\not \partial)^{n} \psi\right\rangle$ by $m^{n}\langle\bar{\psi} \psi\rangle+O(g)$ produces such a cancellation. However, the OPE is surely not valid at the scale of the $u$ or $d$ constituent masses and, even if were we to accept such calculations as order of magnitude estimates, what we would really like is a physically meaningful, gauge invariant running mass for the dressed quarks. The constituent masses obtained from hadronic spectroscopy will then be formed from some average over the effective masses of the quarks inside the hadrons. The rest of this section describes a first step in this direction.

The leading order OPE correction to the physical propagator comes from calculating the coefficient of $\langle\bar{\psi} \psi\rangle$ at order $m^{0}$ and $\alpha_{s}$. This may, in close analogy to the perturbative calculation of the first half of this section, be found from the diagrams of Fig. 6.2. We work in a general Lorentz gauge. It is natural to divide the calculation into two steps: firstly we calculate the terms which depend upon the gauge parameter in the diagrams and, after we have satisfied ourselves that their sum vanishes, we calculate the gauge-independent OPE correction.

Since we are calculating the entire propagator at lowest order in the current quark mass, we have to expand the external propagators (in Fig.'s 6.2a, c and d) in the mass. Higher order condensates, of the form $\left\langle\bar{\psi}(\not \partial)^{n} \psi\right\rangle$, can be related via the equation of motion to $m^{n}\langle\bar{\psi} \psi\rangle+O(g)$ and should be introduced in order to investigate the gauge independence of these corrections. We will not study such terms here. The lowest order calculation is completely standard and the perturbative gluon propagator in the three diagrams which receive a contribution from the gluon cloud around the quark is just as in the first part of this section.

Each of the diagrams in Fig. 6.2 includes a term linear in the gauge parameter $\xi$. To lowest order in $m$ we find that the magnitudes of all four terms are identical and the sum in the dressed propagator vanishes. This shows that the leading OPE correction is, as we had hoped, gauge-invariant.

The remainder may be found by evaluating each of the diagrams in Feynman gauge. The result may be readily checked to be:

$$
S_{F}\left(p^{2}\right)=\frac{1}{\not p}+\frac{\left(N_{c}^{2}-1\right) \pi \alpha_{s}\langle\bar{\psi} \psi\rangle}{2 N_{c}^{2} p^{4}}\left[\left(D-1-\frac{p_{0}^{2}}{\boldsymbol{p}^{2}}\right)\right],
$$


which result has been previously obtained ${ }^{[79]}$ as the leading OPE correction to the Coulomb gauge quark propagator ${ }^{9}$. In the instantaneous approximation this is just the standard Landau gauge result.

Summing up this Coulomb gauge result we have:

$$
S_{F}\left(p^{2}\right)=\frac{1}{p-\Sigma\left(p_{0}^{2}, \boldsymbol{p}^{2}\right)},
$$

where $\Sigma$ may be read off from (6.22).

We now have to interpret this result. Although (6.23) seems to vanish for a static quark, which would be a further signal of confinement, we should recall that the OPE is only valid in the deep-Euclidean region, i.e., for large $\boldsymbol{p}^{2}$. What we can, however, say from (6.22) and (6.23) is that OPE effects from the quark condensate introduce gauge-invariant, non-perturbative corrections in the dressed quark propagator which have a power-like fall off and introduce a running mass scale. We see also from $\Sigma$ in (6.23) that at Euclidean momenta, where we may trust the OPE, the sign of the self energy is that of a (running) positive mass and that the scale which the non-perturbative effects have introduced is of the order of the light quark constituent masses. Even if we do not want to extrapolate (6.23) down to small momenta, we find the appearance of this constituent mass scale (and its gauge invariance) highly suggestive.

We should perhaps conclude this section by remarking ${ }^{[0]}$ that for heavy quarks the fermionic condensate is small, $\langle\bar{Q} Q\rangle \approx-\frac{1}{12}\left\langle\frac{\alpha_{s}}{\pi} G^{2}\right\rangle / m_{Q}$, where $\left\langle\frac{\alpha_{s}}{\pi} G^{2}\right\rangle$ is the gluon condensate $\left(\approx(360 \mathrm{MeV})^{4}\right.$. This means that any change in the heavy quark masses from the condensate will be very small. Higher dimensional condensates will be power suppressed and typical condensate scales are very small compared to the heavy quark masses. This implies that the heavy quark current and constituent masses are very similar.

The calculations of this section raise several immediate questions. Clearly it is interesting to see how far we can take this OPE approach. In addition one would like to see to what extent the incorporation of such non-perturbative effects as instantons is feasible. As we proved in Sect. 5, a full non-perturbative description of the dressing is impossible. However, although studying the breakdown of our construction for a single quark is important, it is perhaps more physical to study the limitations of the dressing in quark-antiquark and three quark colour singlet systems - since these states have a well-defined hadronic description. The next section will therefore be devoted to the simplest such system: a meson made up of two ultra-heavy quarks.

9 A factor of 3 is misprinted in that reference. 


\section{The Potential Between Two Heavy Quarks}

Wilson ${ }^{[81]}$ has suggested that studying a non-local operator whose behaviour in a certain limit yields the static fermion-antifermion potential will give the confinement scale. This approach to the potential has been widely studied: in perturbation theory this Wilson loop has been calculated ${ }^{[82]}$ to $O\left(g^{6}\right)$ and on the lattice it has been extensively investigated. We will reconsider this approach in the light of our construction in the latter part of this section and argue that a very similar operator may be constructed out of dressed fields. This will give us one relatively familiar route to the confinement scale (signalled by the, as we have shown, inevitable breakdown of the dressing). We now, however, wish to directly calculate the potential between two dressed fermions in perturbation theory. For QED this will give the Coulomb potential. In QCD we will see that our approach involves, to any given order in the coupling, calculations which are one loop less than the corresponding Wilson loop approach. This further shows the utility of the dressed quarks presented in this paper and opens the door to higher order calculations of the potential than have yet been performed.

Recalling Dirac's original motivation for introducing the dressing, as discussed in Sect. 4, we can write the electric field associated with the static fermion as

$$
E_{i}^{a}(x)\left|\psi_{\mathrm{c}}(y)\right\rangle=\left[E_{i}^{a}(x), \psi_{\mathrm{c}}(y)\right]|0\rangle,
$$

where $\left|\psi_{\mathrm{c}}(y)\right\rangle=\psi_{\mathrm{c}}(y)|0\rangle$. Here we assume that $E_{i}^{a}(x)|0\rangle=0$, which is valid in this static situation. For two charges the electric field may be analogously shown to be

$$
E_{i}^{a}(x)\left|\bar{\psi}_{\mathrm{c}}(y) \psi_{\mathrm{c}}\left(y^{\prime}\right)\right\rangle=\left(\left[E_{i}^{a}(x), \bar{\psi}_{\mathrm{c}}(y)\right] \psi_{\mathrm{c}}\left(y^{\prime}\right)+\bar{\psi}_{\mathrm{c}}(y)\left[E_{i}^{a}(x), \bar{\psi}_{\mathrm{c}}\left(y^{\prime}\right)\right]\right)|0\rangle,
$$

where now $\left|\bar{\psi}_{\mathrm{c}}(y) \psi_{\mathrm{c}}\left(y^{\prime}\right)\right\rangle=\bar{\psi}_{\mathrm{c}}(y) \psi_{\mathrm{c}}\left(y^{\prime}\right)|0\rangle$. Given that both of these dressings correspond to static (ultra-heavy) charges, which have no magnetic fields, the appropriate measure of the energy of this configuration is determined by the Hamiltonian ${ }^{10}$

$$
H=\frac{1}{2} \int d^{3} x E_{i}^{a}(x) E_{i}^{a}(x) .
$$

10 The Hamiltonian appropriate to Lorentz gauges can be written as $H=\frac{1}{2} \int d^{3} x\left(E^{2}+B^{2}\right)+\left[Q_{\mathrm{BRST}}, \Psi\right]$, where $Q_{\mathrm{BRST}}$ is the BRST charge and $\Psi$ is a ghost number minus one function which contains all the information about the gauge fixing ${ }^{[83]}$. The vacuum and the two particle dressed states needed here are constructed so as to be BRST invariant, hence the commutator term in the Hamiltonian does not contribute to the potential - effectively we can use $H=\frac{1}{2} \int d^{3} x\left(E^{2}+B^{2}\right)$. Now our static dressing does not generate a magnetic field, so the static interfermion potential is determined by the electric field part of this Hamiltonian. Had we chosen to work in Coulomb gauge, the dressing would have vanished, but the above argument for the form of the effective Hamiltonian does not go through ${ }^{[84]}$. 
The energy can be divided into a self-energy part and the intercharge potential. This last part depends upon the separation of the charges, we now wish to calculate this. For simplicity we start with the QED case.

In the abelian theory we find using (4.6) that

$$
E_{i}(x)\left|\bar{\psi}_{\mathrm{c}}(y) \psi_{\mathrm{c}}\left(y^{\prime}\right)\right\rangle=\frac{e}{4 \pi}\left(\partial_{i} \frac{1}{|\boldsymbol{y}-\boldsymbol{x}|}-\partial_{i} \frac{1}{\left|\boldsymbol{y}^{\prime}-\boldsymbol{x}\right|}\right)\left|\bar{\psi}_{\mathrm{c}}(y) \psi_{\mathrm{c}}\left(y^{\prime}\right)\right\rangle \text {. }
$$

Thus we obtain the expected result for the potential between such heavy fermions

$$
\left\langle\bar{\psi}_{\mathrm{c}}(y) \psi_{\mathrm{c}}\left(y^{\prime}\right)|H| \bar{\psi}_{\mathrm{c}}(y) \psi_{\mathrm{c}}\left(y^{\prime}\right)\right\rangle=-\frac{e^{2}}{4 \pi} \frac{1}{\left|\boldsymbol{y}-\boldsymbol{y}^{\prime}\right|},
$$

where we have dropped the divergent self-energy terms.

In QCD the order $g^{2}$ calculation mirrors the previous argument. Note, however, that with our conventions for the $T^{a}$ matrices the trace $\operatorname{tr} T^{a} T^{b}$ is negative definite. One thus obtains the QED result once more, but with the correct colour prefactor. This simple calculation has, in contrast to the more familiar Wilson loop technique, not involved the vector boson propagator! It seems that the dressing has directly incorporated these contributions. We will now sketch the argument that such simplifications are present at all orders in this approach to the potential.

The explicit static dressing found above is generically of the form

$$
h \sim 1+g A+g^{2} A^{2}+g^{3} A^{3}+\cdots+g^{n} A^{n}+\cdots,
$$

where by $A^{r}$ we simply denote the power of the vector potential in any expression. Thus, following our above line of argument, we see that such a dressing contributes an electric field of the form

$$
E \sim g+g^{2} A+g^{3} A^{2}+\cdots
$$

The energy of a static quark-antiquark system will then be of the form

$$
\left\langle\bar{\psi}_{\mathrm{c}} \psi_{\mathrm{c}}\left|E^{2}\right| \bar{\psi}_{\mathrm{c}} \psi_{\mathrm{c}}\right\rangle \sim g^{2}\langle 0|1| 0\rangle+g^{4}\left\langle 0\left|A^{2}\right| 0\right\rangle+g^{6}\left\langle 0\left|A^{4}\right| 0\right\rangle+\cdots
$$

The first term in this schematic series is just our above calculation of the Coulombic potential. The next order in the potential involves the calculation of $\left\langle 0\left|A^{2}\right| 0\right\rangle$ to order $g^{0}$, i.e., the free propagator. The free propagator of a general Lorentz gauge may be employed due to the gauge invariance arguments mustered above. The form of the dressing 
dictates the various projections of the propagator that enter into the calculation of the $g^{4}$ potential. This term in the potential can in the Wilson loop scheme be found from a one loop perturbative calculation which is then nested inside the contour of the loop ${ }^{11}$. We see that the dressing transforms a one loop calculation into an algebraic one involving only the free propagator.

This is not to say that the still higher order calculation of the potential remains purely algebraic in this scheme. At order $g^{6}$, which is the highest order term extracted from the Wilson loop method till now, we have two contributions: one from the $g^{6} A^{4}$ term in (7.8), which involves a tree level four point function, and a second from the $g^{4} A^{2}$ term where now, rather than the free propagator, the $g^{2}$ term in the vector propagator appears. Again this involves a perturbative calculation one loop lower than its Wilson loop counterpart.

We thus see that the approach to the interquark potential detailed here involves significantly less complicated integrals than the conventional one. That the projections due to the dressing can be algebraically involved is by comparison of little importance. We believe the $O\left(g^{8}\right)$ term in the static potential is now quite feasible - the most complicated term being projections of the two loop, Lorentz gauge propagator.

Our philosophy in this paper has been to use dressed quarks as fundamental entities insofar as this is possible. We have seen above that we can discuss the perturbative interquark potential in this fashion. Cahill and Stump ${ }^{[94]}$ considered in an interesting paper the potential from a different perspective. These authors introduced a cloud of glue to dress a non-local quark-antiquark pair corresponding to an ultra-heavy meson. This glue was such that the resulting system was gauge invariant, i.e., they considered

$$
\bar{\psi}_{\mathrm{c}}(y) K\left(y, y^{\prime}\right) \psi_{\mathrm{c}}\left(y^{\prime}\right)
$$

where, to ensure gauge invariance, the field dependent ansatz for $K$ had to transform under a gauge transformation as $K \rightarrow U^{-1}(y) K U\left(y^{\prime}\right)$. These authors argued that a Wilson line was too singular to be a good ansatz for the physical cloud of glue. Using energy minimisation arguments they then arrived at a formula for $K$ which yielded the usual potential to order $g^{4}$. Upon examining their formulae we can recognise that the gluonic cloud used in their paper is a simple product of our static quark dressing

$$
K\left(y, y^{\prime}\right)=h_{\mathrm{c}}(y) h_{\mathrm{c}}^{-1}\left(y^{\prime}\right)
$$

11 The level of complexity of this perturbative calculation is such that it is the most widespread diagnostic tool for prescriptions for dealing with the singularity in the axial gauge propagator ${ }^{[68]}$. 
which as we have seen gives a gauge invariant description of such a meson. Combining their analysis with ours we arrive at another characterization of confinement: we can no longer talk about quarks at the scale from which the mesonic glue does not factor into a product of two, individual constituent glue factors.

Given the widespread use of the Wilson loop method, it is natural to try to reformulate it in terms of the dressed quark picture. We therefore now recap the essential details of that approach. Consider a pair of heavy fermions connected by a gluonic string along some path, $\Gamma$. We will assume that the path $\Gamma$ lies in the time slice of the fermions but is otherwise arbitrary. We now define the state

$$
|\Gamma, t\rangle \equiv \bar{\psi}(\boldsymbol{x}, t) P \exp \left(i \int_{\boldsymbol{x}}^{\boldsymbol{y}} d z_{i} A^{i}(\boldsymbol{z})\right) \psi(\boldsymbol{y}, t)|0\rangle,
$$

where the integral is along $\Gamma$ and $P$ implies a path ordered exponential. The overlap of two such states at time $t=0$ and $t=T$ yields for large (Euclidean) $T$

$$
\begin{aligned}
\lim _{T \rightarrow \infty} M(T, \Gamma) & \equiv\langle\Gamma, 0 \mid \Gamma, T\rangle \\
& =\left\langle\Gamma, 0\left|e^{-H T}\right| \Gamma, 0\right\rangle \\
& \sim e^{-E_{0} T},
\end{aligned}
$$

where we have inserted a complete set of energy eigenstates. We see that only the lowest energy eigenstate, with energy $E_{0}$, contributes for very large $T$. Since we will restrict our attention to static quarks, (7.11) should have non-vanishing overlap with such mesonic states where the two fermions are situated at $\boldsymbol{x}$ and $\boldsymbol{y}$, in consequence we can use (7.12) to extract the dependence of the mesonic ground state energy upon the quark-antiquark separation, $R$. This should be independent of the particular path, $\Gamma$, chosen to link the quarks (although a string whose path, in units of typical hadronic scales, extended over a very large distance away from the quarks would presumably have a miniscule overlap with such a meson). We may now apply standard approaches to rewrite (7.12) as a Wilson loop.

Consider the following evolution amplitude

$$
\begin{aligned}
& M(T, \Gamma) \equiv\langle 0| \bar{\psi}(\boldsymbol{x}, 0) P \exp \left(i \int_{\boldsymbol{x}}^{\boldsymbol{y}} d z_{i} A^{i}(\boldsymbol{z}, 0)\right) \psi(\boldsymbol{y}, 0) \times \\
& \bar{\psi}(\boldsymbol{x}, T) P \exp \left(i \int_{\boldsymbol{x}}^{\boldsymbol{y}} d z_{i} A^{i}(\boldsymbol{z}, T)\right) \psi(\boldsymbol{y}, T)|0\rangle .
\end{aligned}
$$

The static Wilson loop is now obtained in three steps: firstly the fermion fields (or at least the flavour involved in Eq.7.13) are integrated out, this replaces the fermions by 
propagators in a background field and a determinant which is unity if we ignore closed fermion loops (quenched approximation). Some of these contributions correspond to pair creation and annihilation diagrams which we ignore since we want to study the potential. The next step is to consider the propagators in the static approximation as was first done by Brown and Weisberger ${ }^{[85]}$ who made the gauge invariant approximation that the quark two-point Green's function obeys

$$
\left(\gamma_{0} D^{0}-m\right) S_{0}(x, y)=\delta^{(4)}(x-y)
$$

where $S_{0}$ is the static propagator in a background field. (The solution to this equation contains a factor of $\delta^{(3)}(\mathbf{x}-\boldsymbol{y})$.) Details of all these steps may be found in a lucid review by Gromes ${ }^{[86]}$. Since the quarks do not move they have potential but no kinetic energy and this is what the overlap (7.12) measures. The upshot is that the above evolution amplitude may be written as a gauge invariant expectation value of a loop operator

$$
M(T, \Gamma)=\langle\operatorname{tr} P \exp (i g \oint d z \cdot A(z))\rangle,
$$

where the loop runs over the path $\Gamma$ in the initial time slice, then over a straight line from $(\boldsymbol{y}, 0)$ to $(\boldsymbol{y}, T)$, then back over the path $\Gamma$ (but now at time $T$ ) and then directly down from $(\boldsymbol{x}, T)$ to $(\boldsymbol{x}, 0)$, which completes the loop. For the particular case that $\Gamma$ is taken to be the straight line joining $\boldsymbol{x}$ and $\boldsymbol{y}$ one then has the standard, rectangular Wilson loop.

At lowest order in perturbation theory the potential also displays a Coulombic $1 / R$ fall off ${ }^{[87]}$. Numerical studies, primarily on the lattice, are widely employed to study the potential between two static sources at larger separations ${ }^{[8]}$. It appears that the results are consistent with a combination of a Coulombic and a linearly rising term, which should yield confinement. Variations around the straight line base can be used to test the accuracy of numerical results for the potential. It is found in lattice simulations that 'smearing' out the line improves the numerical accuracy ${ }^{[88]}$. In this context see also Ref. 89.

We may now consider studying the interaction between a dressed quark and a dressed antiquark in the above spirit. We take a static quark and a static antiquark at $\boldsymbol{x}$ and $\boldsymbol{y}$, each dressed as discussed in Sect.'s 4 and 5. The following overlap

$$
M_{\mathrm{c}}(T, \boldsymbol{x}-\boldsymbol{y}) \equiv\left\langle\bar{\psi}_{\mathrm{c}}(0, \boldsymbol{x}) \psi_{\mathrm{c}}(0, \boldsymbol{y}) \mid \bar{\psi}_{\mathrm{c}}(T, \boldsymbol{x}) \psi_{\mathrm{c}}(T, \boldsymbol{y})\right\rangle
$$

where

$$
\left|\bar{\psi}_{\mathrm{c}}(t, \boldsymbol{x}) \psi_{\mathrm{c}}(t, \boldsymbol{y})\right\rangle \equiv \bar{\psi}_{\mathrm{c}}(t, \boldsymbol{x}) \psi_{\mathrm{c}}(t, \boldsymbol{y})|0\rangle
$$


gives, for large $T$, the energy of the lowest mesonic state, as such it is analogous to the more traditional Wilson loop.

In analogy to the derivation of the Wilson loop we integrate out the fermions to obtain two propagators (and drop the terms that correspond to creation-annihilation effects). In the static approximation we can, in analogy to Brown and Weisberger ${ }^{[85]}$, replace the propagators by path ordered exponentials along straight lines evolving in time. These lines are themselves gauge invariant except at their ends, but this gauge dependence is annulled by the four gluonic clouds centred around these ends. The study of such an object as (7.16) should at small distances, where the perturbative definition of the static dressed quarks is reliable, yield the usual potential between two static quarks. At larger distances, however, the potential should become non-perturbative and confining effects will dominate. Since (7.16) is only perturbatively gauge invariant — as we saw in Sect. 5 no full solution for the dressing exists - for large enough separations gauge dependent effects eventually will become visible in the potential as it may be calculated from (7.16). This explicit breakdown of the quark picture will correspond to the confinement scale.

The calculation of the potential between dressed quarks involves, as well as the nonlocality inherent in the lines, the non-local gluonic dressings at the ends of the lines. These last may be removed, however, by working in Coulomb gauge. It may be quickly seen that at one loop this then yields a Coulomb potential between the quarks as usual. This agreement with the potential from the static Wilson loop is heartening if not unexpected: the end rungs of the Wilson loop rectangle should be unimportant in the large $T$ limit at this level (see, e.g., Ref. 87). The question we are faced with is where should this agreement break down? It has been argued by Fischler ${ }^{[82]}$ that the ends of the static Wilson loop are only of importance for large fields. This would imply that the gluonic clouds around the ends of the lines are first important when non-perturbative effects are considered. A numerical study on the lattice of where the Gribov problem first raises its head in the potential between the dressed quarks should be able to measure this. One difficulty with such a simulation is raised by the non-local gluonic clouds present in the two end time slices. By working in the Coulomb gauge one can remove the non-locality and also not have to worry about the exact form of the cloud (for examples of Coulomb gauge lattice investigations see, e.g., Ref.'s 90 and 91). The appearance of the Gribov ambiguity for larger separations would herald confinement. We would urge that more work be done on understanding the Gribov ambiguity on the lattice ${ }^{[92]}$. We note also in this context 
that it seems that many, perhaps most, of these gauge ambiguities as they are seen in numerical simulations seem to be lattice artefacts ${ }^{[93]}$ which have no physical meaning. The breakdown of a quark picture is then to be established from the onset of non-artefact Gribov ambiguities.

The extension of both the above approaches to baryons, glueballs and exotics is more or less direct. Consider, for example, having three static dressed quarks. The energy of the lowest lying heavy baryon may be straightforwardly found, in analogy to (7.8) or (7.16). The usual Wilson loop picture for this has been studied on the lattice ${ }^{[95]}$. Here one considered three static quarks connected by strings to some central point. The results of the numerical simulations seem to indicate that the lattices only represent small volumes. (Essentially because the resulting potential was of a form expressible as a sum over two body forces alone, see also Ref. 86.) Long distance physics, and quark confinement in baryons, would thus appear to require better numerical simulations.

One can also consider extending the above considerations to exotic particles and glueballs, which are much more poorly experimentally understood. One can here, e.g., use a globally colourless configuration of gauge invariant, dressed gluons (such as the solutions found in Sect. 5). We refer to various discussions of constituent gluons ${ }^{[96,97]}$.

We have now seen that the dressings lead to an efficient method of calculating the perturbative potential between static charges. Furthermore, we have suggested various ways of obtaining the confinement scale within this framework. In the real world we have, of course, light quarks and we must therefore go beyond the static approximation. This will be the subject of the rest of this paper. 


\section{Lorentz Transformations in Gauge Theories}

In order to go beyond our previous description of solely static quarks, we need to address the issue of how Lorentz transformations must be implemented in gauge theories. We will show that requiring the Lorentz generators to be gauge invariant modifies their action on charged states. For simplicity, this treatment will mainly be carried out in the context of QED - it will be clear from our presentation that much the same conclusions can be reached for any gauge theory. We shall show that, even in such an abelian theory, the action of the Lorentz group is sensitive to whether ghosts are introduced or not. Important consequences of our results for the construction of non-static dressings will be discussed in the next section, where we will give explicit solutions for the dressing function of a moving charge.

In Sect. 3 an argument for the breakdown of the expected action of the Lorentz group was presented. Till now, this surprising aspect of gauge theories has played a minor role in our presentation of charged sectors: its only implication being as another reason for lowering our naive expectation of manifest Lorentz invariance in the dressing and related gauge fixing. We have now, though, reached the stage in our account of constituent quarks where we want to demonstrate that the static dressing can be extended to a more dynamical one. Hence we need to make clear how Lorentz transformations are to act on such fields.

In order to make this discussion easier to follow, let us first summarize what we are going to show. There are two important issues that we will address: firstly, how should Lorentz transformations act on the local fields in a gauge theory (for obvious practical reasons we include here the gauge non-invariant ones such as the potential); secondly, how should they act on non-local (charged) fields?

By starting with the standard QED Lagrangian we will demonstrate that the requirement of gauge invariance modifies the action of the Lorentz generators in a profound way. On general fields this modification is sufficiently drastic to obstruct them from carrying a representation of the Lorentz group. However, on local observables the modified generators will have the same action as the usual ones, and hence they will carry the expected representations of the Lorentz group. The interesting point, though, is that on charged (and hence non-local but gauge invariant) fields the action of these generators is modified. As we will see in Sect. 9, this observation will have important consequences for our account of such charged states and, in particular, for our description of the light constituent quarks. 
It is also possible, but usually not considered very useful, to introduce ghosts into QED. By doing this we will then demonstrate the attractive result that by insisting on BRST invariance, the standard action of the Lorentz group on all local fields is recovered - only in the nonlocal charged sectors is this not the case.

The QED Lagrangian is

$$
\mathcal{L}_{\mathrm{QED}}=-\frac{1}{4} F_{\mu \nu} F^{\mu \nu}+i \bar{\psi} \gamma^{\mu}\left(\partial_{\mu}+i e A_{\mu}\right) \psi-m \bar{\psi} \psi
$$

In this the potential $A_{\mu}$ is taken to be a four vector and $\psi$ a spinor. Under the infinitesimal Lorentz transformation

$$
x^{\mu} \rightarrow x^{\prime \mu}:=x^{\mu}-f^{\mu},
$$

where $f^{\mu}=-\epsilon^{\mu \nu} x_{\nu}$, the potential thus transforms as ${ }^{[34,98]}$

$$
\delta_{f} A_{\mu}=f^{\alpha} \partial_{\alpha} A_{\mu}+\left(\partial_{\mu} f^{\alpha}\right) A_{\alpha}
$$

The infinitesimal transformations on $\psi$ and $\bar{\psi}$ are then taken to be

$$
\begin{aligned}
& \delta_{f} \psi=f^{\alpha} \partial_{\alpha} \psi+\frac{i}{4} \sigma_{\mu \nu}\left(\partial^{\nu} f^{\mu}\right) \psi \\
& \delta_{f} \bar{\psi}=f^{\alpha} \partial_{\alpha} \bar{\psi}-\frac{i}{4}\left(\partial^{\nu} f^{\mu}\right) \bar{\psi} \sigma_{\mu \nu}
\end{aligned}
$$

where $\sigma_{\mu \nu}=\frac{i}{2}\left[\gamma_{\mu}, \gamma_{\nu}\right]$. Even at this stage, we see an unnaturalness in these transformations as they do not reflect the fact that we are dealing with a gauge theory. In particular, the occurrence of derivatives in (8.4) is at odds with the normal expectation that by making the gauge symmetry local, derivatives on $\psi$ should be replaced by covariant derivatives. As a consequence, if we have two gauge related fermions, $\psi$ and $e^{-i e \Lambda} \psi$, then under the Lorentz transformations they are no longer gauge related! Explicitly:

$$
e^{-i e \Lambda}\left(\psi+\delta_{f} \psi\right) \neq e^{-i e \Lambda} \psi+\delta_{f}\left(e^{-i e \Lambda} \psi\right)
$$

This should be contrasted with the transformation properties of the vector potential where two gauge related potentials are still gauge related after a Lorentz transformation. This observation highlights the fact discussed in Sect. 3 that it is in the charged sector where more care is needed when implementing the Lorentz transformations. To make this unease more precise, and to see how to resolve this problem, we need to further investigate how the Lorentz transformations are generated in QED. 
The invariance of the QED Lagrangian under the Lorentz transformations (8.3) and (8.4) allows us to use Noether's theorem to obtain their generators. Under these transformations the Lagrangian transforms by a total derivative

$$
\delta_{f} \mathcal{L}_{\mathrm{QED}}=\partial_{\mu}\left(f^{\mu} \mathcal{L}_{\mathrm{QED}}\right)
$$

The associated conserved current is then:

$$
\begin{aligned}
M^{\mu} & =\frac{\delta \mathcal{L}_{\mathrm{QED}}}{\delta\left(\partial_{\mu} A_{\nu}\right)} \delta_{f} A_{\nu}-\frac{\delta \mathcal{L}_{\mathrm{QED}}}{\delta\left(\partial_{\mu} \psi\right)} \delta_{f} \psi-f^{\mu} \mathcal{L}_{\mathrm{QED}} \\
& =\frac{1}{2} \epsilon_{\alpha \beta} M^{\mu \alpha \beta}
\end{aligned}
$$

where

$$
M^{\mu \alpha \beta}=x^{\alpha} T_{\mathrm{c}}^{\mu \beta}-T_{\mathrm{c}}^{\mu \alpha} x^{\beta}+A^{\alpha} F^{\mu \beta}-F^{\mu \alpha} A^{\beta}-\frac{1}{2} \bar{\psi} \gamma^{\mu} \sigma^{\alpha \beta} \psi,
$$

and $T_{\mathrm{c}}^{\mu \nu}$ is the canonical stress-energy tensor ${ }^{[99]}$.

$$
T_{\mathrm{c}}^{\mu \nu}=i \bar{\psi} \gamma^{\mu} \partial^{\nu} \psi-F^{\mu \rho} \partial^{\nu} A_{\rho}+\frac{1}{4} g^{\mu \nu} F_{\rho \lambda} F^{\rho \lambda} .
$$

The generators of the Lorentz transformations are then identified with the charges

$$
M^{\alpha \beta}=\int d^{3} x M^{0 \alpha \beta} .
$$

Using (3.19) and (3.20) one finds that

$$
\begin{aligned}
{\left[i M_{\alpha \beta}, A_{i}\right] } & =\left(x_{\alpha} \partial_{\beta}-x_{\beta} \partial_{\alpha}\right) A_{i}+g_{i \alpha} A_{\beta}-g_{i \beta} A_{\alpha} \\
{\left[i M_{\alpha \beta}, \psi\right] } & =\left(x_{\alpha} \partial_{\beta}-x_{\beta} \partial_{\alpha}\right) \psi-\frac{i}{2} \sigma_{\alpha \beta} \psi
\end{aligned}
$$

which, when multiplied by $\frac{1}{2} \epsilon^{\alpha \beta}$, recovers $\delta_{f} A_{i}$ and $\delta_{f} \psi$. (Just as we saw for gauge invariance, we do not recover how $A_{0}$ transforms as it is not a dynamical field in Eq. 8.1. We will see later in this section how to improve upon this.)

The commutator algebra of these generators closes on itself, i.e., it only involves linear combinations of the generators:

$$
\left[M_{\alpha \beta}, M_{\rho \sigma}\right]=i g_{\alpha \sigma} M_{\beta \rho}-i g_{\alpha \rho} M_{\beta \sigma}+i g_{\beta \rho} M_{\alpha \sigma}-i g_{\beta \sigma} M_{\alpha \rho} .
$$

This tells us that they form a Lie algebra ${ }^{[100]}$ and hence that these are the generators for a unitary implementation of the Lorentz group on these fields. That is, under a finite 
Lorentz transformation described by the matrix $\Lambda$, where to lowest order $\Lambda_{\beta}^{\alpha}=\delta_{\beta}^{\alpha}+\epsilon_{\beta}^{\alpha}$, we have

$$
\begin{aligned}
A_{\mu}^{\prime}(x) & =U(\Lambda) A_{\mu}(x) U^{-1}(\Lambda) \\
\psi^{\prime}(x) & =U(\Lambda) \psi(x) U^{-1}(\Lambda)
\end{aligned}
$$

where the unitary operator $U(\Lambda)$ representing the Lorentz transformation is given by

$$
U(\Lambda)=e^{\frac{i}{2} M_{\alpha \beta} \epsilon^{\alpha \beta}} .
$$

Now the difficulties with this account of Lorentz invariance in such a gauge theory become clear: from (8.8) and (8.9) we can see that the generators are not gauge invariant and hence cannot be identified with observables. This means that we have the former of the alternatives discussed in Sect. 3: the Lorentz group is implemented unitarily on all the local fields but it is not generated by observables, as such it has no clear action on the physical (gauge invariant) states or observables.

There is, however, a standard prescription for improving the stress-energy tensor (8.9) which, as a consequence, makes it gauge invariant ${ }^{[99]}$. That is, we consider the gauge invariant stress-energy tensor ${ }^{12}$

$$
T^{\mu \nu}=i \bar{\psi} \gamma^{\mu} D^{\nu} \psi+F^{\mu \rho} F_{\rho}{ }^{\nu}+\frac{1}{4} g^{\mu \nu} F_{\rho \lambda} F^{\rho \lambda} .
$$

This can be viewed as an addition to the canonical stress-energy tensor

$$
T^{\mu \nu}=T_{\mathrm{c}}^{\mu \nu}+F^{\mu \rho} \partial_{\rho} A^{\nu}-e \bar{\psi} \gamma^{\mu} A^{\nu} \psi
$$

The argument for why we are free to add the extra terms is that, after using the equation of motion, $\partial_{\rho} F^{\mu \rho}=-e \bar{\psi} \gamma^{\mu} \psi$, they can be written as a divergence

$$
T^{\mu \nu}=T_{\mathrm{c}}^{\mu \nu}+\partial_{\rho}\left(X^{\rho \mu \nu}\right)
$$

12 The standard prescription actually aims to make the stress-energy tensor symmetric in $\mu$ and $\nu$, which is a stronger condition than just making it gauge invariant. To further improve our expression for the stress-energy tensor so that it is symmetric the term $\partial_{\rho}\left(F^{\mu \rho} A^{\nu}-\frac{1}{4} \bar{\psi}\left(\gamma^{\nu} \sigma^{\rho \mu}+\gamma^{\mu} \sigma^{\rho \nu}-\gamma^{\rho} \sigma^{\mu \nu}\right) \psi\right)$ must be added to the canonical stress-energy tensor. This additional term does not alter our discussion of the generators, and for simplicity we avoid this complication. We note, though, that if we wished to couple to gravity then the symmetric form of the stress-energy tensor must be used. 
where $X^{\rho \mu \nu}=F^{\mu \rho} A^{\nu}$ is anti-symmetric in $\rho$ and $\mu$. This last property ensures that the gauge invariant stress-energy tensor (8.17) is conserved as long as the canonical one is.

The same construction can be applied to the Lorentz generators. We seek a modification

$$
\bar{M}^{\mu \alpha \beta}=M^{\mu \alpha \beta}+\partial_{\rho} X^{\rho \mu \alpha \beta},
$$

such that the resulting charge is gauge invariant and conserved. One finds that if we take

$$
X^{\rho \mu \alpha \beta}=x^{\alpha} X^{\rho \mu \beta}-X^{\rho \mu \alpha} x^{\beta},
$$

then the gauge invariant current is given by

$$
\bar{M}^{\mu \alpha \beta}=x^{\alpha} T^{\mu \beta}-T^{\mu \alpha} x^{\beta}-\frac{1}{2} \bar{\psi} \gamma^{\mu} \sigma^{\alpha \beta} \psi
$$

It is usually argued that such modifications do not effect the action or algebra of the charges since it amounts to adding a total divergence to the generators. However, care is needed since we have seen that charges imply that surface terms cannot always be neglected. Also the equations of motion have been used in getting to the gauge invariant description (8.22); so again, care is needed.

As it is by construction conserved, this gauge invariant charge will generate symmetries of the theory. It is clearly of great interest to know how they are related to the original Lorentz transformations. One finds for example that

$$
\left[i \bar{M}_{\alpha \beta}, A_{i}\right]=x_{\alpha} F_{\beta i}-x_{\beta} F_{\alpha i}
$$

and

$$
\left[i \bar{M}_{\alpha \beta}, \psi\right]=\left(x_{\alpha} D_{\beta}-x_{\beta} D_{\alpha}\right) \psi-\frac{i}{2} \sigma_{\alpha \beta} \psi
$$

Multiplying by $\frac{1}{2} \epsilon^{\alpha \beta}$ these give the gauge covariant Lorentz transformations ${ }^{[101]}$ on $A_{i}$ and $\psi:$

$$
\begin{aligned}
\bar{\delta}_{f} A_{i} & =f^{\alpha} F_{\alpha i} \\
\bar{\delta}_{f} \psi & =f^{\alpha} D_{\alpha} \psi+\frac{i}{4} \sigma_{\alpha \beta}\left(\partial^{\beta} f^{\alpha}\right) \psi
\end{aligned}
$$

These transformations have many interesting properties and applications ${ }^{[102]}$, and we note that we now have the desired covariant derivatives on the fermionic fields. However, these are not the expected Lorentz transformations. We will now investigate the physical consequences of this. 
It must be emphasised that Lorentz invariance is of central importance only for physical states and observables. As we have repeatedly stressed, gauge theories carry with them a vast amount of unphysical luggage. Thus the fact we have been forced to tinker with the Lorentz generators should not, in itself, cause too much concern - as long as we are clear that we have not altered any physical consequence of the theory. To see this let us first look at the modification to the stress-energy tensor (8.9) brought about by our insistence on gauge invariance.

We recall that the canonical stress-energy tensor (8.9) is used to construct the momentum $P_{\mathrm{c}}^{\nu}=\int d^{3} x T_{\mathrm{c}}^{0 \nu}$. This momentum plays a very privileged role in any formulation of quantum field theory, and one would be loth to see any modification to its role as the generator of spacetime translations. The gauge invariant stress-energy tensor (8.17) gives the momentum

$$
P^{\nu}=P_{\mathrm{c}}^{\nu}+\int d^{3} x\left(F^{0 i} \partial_{i} A^{\nu}-e J^{0} A^{\nu}\right) .
$$

We note that, by integrating by parts, this can be written as

$$
P^{\nu}=P_{\mathrm{c}}^{\nu}-\int d^{3} x G A^{\nu}
$$

where $G=\partial_{i} F^{0 i}+e J^{0}$ is Gauss' law, which we recall is the generator of the gauge transformations. This means that on gauge invariant states and observables we recover the action of the normal momentum as long as we are free to neglect the surface term. The potentially troublesome surface term in this case is $\lim _{R \rightarrow \infty} \int_{S_{R}} d \boldsymbol{s} \cdot \boldsymbol{E} A^{\nu}$. We recall from our discussion in Sect. 3 that the potential is assumed to fall off as $1 / R$ and the electric field as $1 / R^{2}$. These assumptions on the fall off rates of these fields imply that this surface term is zero, so we are free to take the normal momentum action on all gauge invariant states, i.e., on both charged and uncharged states. Let us now repeat this analysis for the Lorentz generators.

From (8.22) we see that

$$
\begin{array}{rlr}
\bar{M}^{\alpha \beta} & =M^{\alpha \beta}+\int d^{3} x\left(F^{0 \alpha} A^{\beta}-F^{0 \beta} A^{\alpha}+F^{0 i}\left(x^{\alpha} \partial_{i} A^{\beta}-x^{\beta} \partial_{i} A^{\alpha}\right)\right. \\
& \left.-e J^{0}\left(x^{\alpha} A^{\beta}-x^{\beta} A^{\alpha}\right)\right) \\
& =M^{\alpha \beta}-\int d^{3} x\left(x^{\alpha} A^{\beta}-x^{\beta} A^{\alpha}\right) G, &
\end{array}
$$

plus the surface term

$$
\lim _{R \rightarrow \infty} \int_{S_{R}} d \boldsymbol{s} \cdot \boldsymbol{E}\left(x^{\alpha} A^{\beta}-x^{\beta} A^{\alpha}\right)
$$


So again, we see that as long as this surface term is zero, the Lorentz transformations on gauge invariant objects are recovered. In particular, on all local chargeless states this will be the case. But what about charged states? Here we see that due to the extra coordinate variable in (8.29), the fall off conditions on the potentials will result in a non-zero surface contribution. To be explicit, for a static charge we have $A_{i}=0$ while $A_{0} \sim 1 / R$, so for boosts, where the generator is $\bar{M}^{0 i}$, this surface term will not vanish.

In summary we see that we recover the action of the Lorentz group on the observables, but on charged states the action is modified. Given that these charged states are not observables, this modification does not lead to any unphysical consequences. However, we shall now demonstrate that there is quite a profound technical complication caused by this since it is not possible to use these infinitesimal transformations to build up finite ones on any charged state in a unitary way!

The reason why the modified generators do not lead to a representation of the Lorentz group is because their commutator algebra no longer closes as in (8.13). Indeed, we can see that

$$
\left[\bar{M}_{\alpha \beta}, \bar{M}_{\rho \sigma}\right]=i g_{\alpha \sigma} \bar{M}_{\beta \rho}-i g_{\alpha \rho} \bar{M}_{\beta \sigma}+i g_{\beta \rho} \bar{M}_{\alpha \sigma}-i g_{\beta \sigma} \bar{M}_{\alpha \rho}+i K_{\alpha \beta \rho \sigma}
$$

Before giving an explicit example for the additional term $K_{\alpha \beta \rho \sigma}$, we note that its addition to the Lorentz algebra means that we cannot exponentiate these generators to build up the action of finite transformations as in (8.16). For brevity we will call $K_{\alpha \beta \rho \sigma}$ the central term for the gauge covariant Lorentz algebra.

The proof of this result is tedious, and we only give a brief sketch here for the simpler case when we just deal with that part of the Lorentz group that deals with rotations ${ }^{13}$. The rotation generators are $\bar{M}_{i j}$. To find the expression for the central term $K_{i j k l}$ in this case we use the Jacobi identity:

$$
\left[i \bar{M}_{i j},\left[i \bar{M}_{k l}, A_{m}\right]\right]-\left[i \bar{M}_{k l},\left[i \bar{M}_{i j}, A_{m}\right]\right]=-\left[\left[\bar{M}_{i j}, \bar{M}_{k l}\right], A_{m}\right]
$$

From (8.23) and (8.30) we find that

$$
\left[i K_{i j k l}, A_{m}\right]=\partial_{m}\left(x_{i} x_{k} F_{l j}-x_{i} x_{l} F_{k j}-x_{j} x_{k} F_{l i}+x_{j} x_{l} F_{k i}\right)
$$

13 One reason for just looking at rotations is that they do not mix the spatial and temporal components of the gauge field (as a boost would). The reason why this is important is that we have, as yet, an incomplete description of the modified Lorentz generators. This is because we are starting with the Lagrangian (8.1) in which $A_{0}$ is just a multiplier field, as such we do not know how these transformations should be extended to $A_{0}$. This will be improved upon below. 
Similarly on $\psi$ we get

$$
\left[i K_{i j k l}, \psi\right]=i e\left(x_{i} x_{k} F_{l j}-x_{i} x_{l} F_{k j}-x_{j} x_{k} F_{l i}+x_{j} x_{l} F_{k i}\right) \psi
$$

From these we deduce that the central term is

$$
\begin{aligned}
K_{i j k l}=\int d^{3} x\left(\partial _ { m } \left(x_{i} x_{k} F_{l j}\right.\right. & \left.-x_{i} x_{l} F_{k j}-x_{j} x_{k} F_{l i}+x_{j} x_{l} F_{k i}\right) E_{m} \\
& \left.-\left(x_{i} x_{k} F_{l j}-x_{i} x_{l} F_{k j}-x_{j} x_{k} F_{l i}+x_{j} x_{l} F_{k i}\right) e \psi^{\dagger} \psi\right)
\end{aligned}
$$

Upon integrating by parts this may be rewritten as a sum of two terms: a part containing Gauss' law

$$
-\int d^{3} x\left(x_{i} x_{k} F_{l j}-x_{i} x_{l} F_{k j}-x_{j} x_{k} F_{l i}+x_{j} x_{l} F_{k i}\right)\left(\partial_{m} E_{m}+e \psi^{\dagger} \psi\right),
$$

and the surface term

$$
\lim _{R \rightarrow \infty} \int_{S_{R}^{2}} d \boldsymbol{s} \cdot \boldsymbol{E}\left(x_{i} x_{k} F_{l j}-x_{i} x_{l} F_{k j}-x_{j} x_{k} F_{l i}+x_{j} x_{l} F_{k i}\right) .
$$

This surface term can be written as

$$
\left(\epsilon_{i j k} \delta_{l s}-\epsilon_{i j l} \delta_{k s}\right) \lim _{R \rightarrow \infty} \int_{S_{R}^{2}} d \boldsymbol{s} \cdot \boldsymbol{E} x_{s} x_{r} B_{r} .
$$

It is now a dynamical problem to determine when this surface term is zero. Clearly for a static charge it vanishes. This should not come as too much of a surprise since the form of the dressing in this case (4.5) is naively rotationally invariant. It is also zero for charges moving with a constant velocity since then the magnetic field satisfies $x_{r} B_{r}=0$. This would suggest that in this abelian theory charges can be rotated in the expected way. It is intriguing to note that the static quark does not appear to have this property.

We have seen that gauge invariance dictates the modifications of the Lorentz generators, a consequence of this is that it is only on the local physical observables that the Lorentz group acts in the expected way. On gauge non-invariant and gauge invariant but charged (and hence non-local) states the action is modified to such an extent that it cannot be implemented unitarily. Although this is clearly physically acceptable, its practical implications are rather unattractive. This analysis, though, is incomplete. As we have stressed earlier, the action on $A_{0}$ has not been determined due to the gauge invariance of (8.1). To remedy this we add a gauge fixing term which breaks the gauge invariance. 
This then reinstates $A_{0}$ as a dynamical variable, but now the requirement that the Lorentz generators are gauge invariant is unnatural (after all, the gauge fixed theory is no longer gauge invariant). To proceed we have to impose the condition that the generators must be BRST invariant. Insisting on BRST invariance in this way puts some restrictions on the form of the effective QED Lagrangian. Let us now discuss this point.

The gauged fixed QED action based now on the abelian version of the Lagrangian (3.33) is BRST invariant, but the Lagrangian itself is not: it picks up a total divergence. We saw the same thing for the Lorentz generators (8.6) and this is quite unobjectionable for a symmetry. (In fact it is mandatory for a spacetime symmetry, but optional for an internal one ${ }^{[99]}$.) However, an invariant stress-energy tensor must be built from an invariant Lagrangian, so we are forced to rewrite (3.33) so that it is strictly invariant under BRST. There are various way to do this, the most attractive is to introduce a scalar field $B$ and take as our effective Lagrangian

$$
\mathcal{L}_{\text {eff }}=\mathcal{L}_{\mathrm{QED}}+\frac{1}{2} B^{2}+\partial_{\mu} B A^{\mu}-i \partial_{\mu} \bar{c} \partial^{\mu} c
$$

The BRST transformations are then: $\delta A_{\mu}=\partial_{\mu} c ; \delta c=0 ; \delta \bar{c}=-i B ; \delta B=0 ; \delta \psi=-i e c \psi$ and $\delta \bar{\psi}=-i e \bar{\psi} c$. We now have, on top of the invariance of (8.38), the nilpotency, $\delta^{2}=0$, which lies at the heart of the BRST method, without use of an equation of motion. We see that the new equations of motion that follow from (8.38) are

$$
\partial_{\mu} F^{\mu \nu}=e \bar{\psi} \gamma^{\mu} \psi-\partial^{\mu} B
$$

and

$$
\partial_{\mu} A^{\mu}=\xi B
$$

We note that $A_{0}$ and $B$ are conjugate variables.

The infinitesimal Lorentz transformations are given by (8.3) and (8.4) supplemented by the scalar transformation rule for the ghosts and $B$, i.e., $\delta_{f} B=f^{\mu} \partial_{\mu} B$ and similarly for $c$ and $\bar{c}$. These result in a canonical stress-energy tensor

$$
\begin{aligned}
T_{\mathrm{c}}^{\mu \nu}=i \bar{\psi} \gamma^{\mu} \partial^{\nu} \psi & -F^{\mu \alpha} \partial^{\nu} A_{\alpha}+\frac{1}{4} g^{\mu \nu} F_{\lambda \rho} F^{\lambda \rho} \\
& +A^{\mu} \partial^{\nu} B-\frac{1}{2} g^{\mu \nu} \xi B^{2}-g^{\mu \nu} \partial_{\lambda} B A^{\lambda} \\
& -i\left(\partial^{\mu} \bar{c} \partial^{\nu} c+\partial^{\nu} \bar{c} \partial^{\mu} c\right)+i g^{\mu \nu} \partial_{\lambda} \bar{c} \partial^{\lambda} c .
\end{aligned}
$$


This is evidently not BRST invariant. To deal with this we improve it by adding the term $\partial_{\lambda}\left(F^{\mu \nu} A^{\nu}\right)$, as we did before. Now after using the equation of motion (8.39) we find the BRST invariant stress-energy tensor

$$
\begin{aligned}
T^{\mu \nu}=i \bar{\psi} \gamma^{\mu} D^{\nu} \psi & +F^{\mu \lambda} F_{\lambda}{ }^{\nu}+\frac{1}{4} g^{\mu \nu} F_{\lambda \rho} F^{\lambda \rho} \\
& +\left(A^{\mu} \partial^{\nu} B+A^{\nu} \partial^{\mu} B\right)-\frac{1}{2} g^{\mu \nu} \xi B^{2}-g^{\mu \nu} \partial_{\lambda} B A^{\lambda} \\
& -i\left(\partial^{\mu} \bar{c} \partial^{\nu} c+\partial^{\nu} \bar{c} \partial^{\mu} c\right)+i g^{\mu \nu} \partial_{\lambda} \bar{c} \partial^{\lambda} c .
\end{aligned}
$$

The same procedure goes through for the Lorentz generators and we end up with the improved, BRST invariant charge (8.22); note that we are now using the above stressenergy tensor.

As before, to investigate the action of this modified generator, we write

$$
\begin{aligned}
\bar{M}^{\alpha \beta}=M^{\alpha \beta}+\int d^{3} x( & F^{0 \alpha} A^{\beta}-F^{0 \beta} A^{\alpha} \\
+ & x^{\alpha}\left(F^{0 i} \partial_{i} A^{\beta}-e J^{0} A^{\beta}+\dot{B} A^{\beta}\right) \\
& \left.\quad-\left(F^{0 i} \partial_{i} A^{\alpha}-e J^{0} A^{\alpha}+\dot{B} A^{\alpha}\right) x^{\beta}\right) .
\end{aligned}
$$

After integrating by parts this gives

$$
\begin{aligned}
& \bar{M}^{\alpha \beta}=M^{\alpha \beta}+\int d^{3} x\left(-\partial_{i} F^{0 i}-e J^{0}+\dot{B}\right)\left(x^{\alpha} A^{\beta}-A^{\alpha} x^{\beta}\right) \\
& +\lim _{R \rightarrow \infty} \int_{S_{R}} d \boldsymbol{s} \cdot \boldsymbol{E}\left(x^{\alpha} A^{\beta}-x^{\beta} A^{\alpha}\right),
\end{aligned}
$$

where we recognise the surface term (8.29) encountered earlier. Note that $\dot{B}$ enters these expressions. This velocity can be replaced using (8.39): $-\partial_{i} F^{0 i}-e J^{0}+\dot{B}=0$. Hence, in marked contrast to what happened without ghosts, we now see that on all local fields, where as we have stated the surface term can be neglected, $\bar{M}^{\alpha \beta}=M^{\alpha \beta}$, and we recover the normal action of the Lorentz group.

We are familiar in non-abelian gauge theories with the introduction of ghosts to maintain unitarity. Our discussion shows that even in the abelian theory, where unitarity is not an issue in covariant gauges, ghosts are essential if one wants to preserve the standard form of the Lorentz generators on local observables and non-observables. A simple argument to see why this is the case comes from the example given earlier, (8.5). Combining the BRST-transformation $\delta \psi=-i e c \psi$ with the Lorentz transformations (8.4) and the scalar transformation rule, $\delta_{f} c=f^{\alpha} \partial_{\alpha} c$, we see that now

$$
\delta\left(\psi+\delta_{f} \psi\right)=\delta \psi+\delta_{f}(\delta \psi)
$$


Hence the reason underlying our initial unease with the Lorentz transformations may be avoided if BRST transformations are used instead of the gauge ones. From the above discussion, though, it is clear that there is still a modification to the Lorentz generators (even in this BRST-formulation) when charges are present. This is because the identification of the modified generators with the standard ones only holds if the surface term in (8.44) may be neglected. We have seen for charged states that this is generally not permitted. In the next section we will see how this is reflected in the construction of dynamical dressings for charged particles. 


\section{Beyond the Static Quark Approximation}

In the constituent quark model a proton, say, contains three light quarks, each of which will be moving. For this reason it is essential to have the correct dressings for non-static quarks. Indeed even in QED the dressings for non-static fermions are needed. In this section we will therefore boost a static charged fermion. This will allow us to find, for the first time, the dressing for a moving charge. Our discussion in the last section implies that care might be needed, and we will see that this is the case. We conclude this section by giving explicit dressings for relativistic and non-relativistic charges.

Up to now we have analysed in detail how a static dressing can be applied to the quark fields, and how this procedure allows us to derive the essential features of the constituent quark model from QCD. The existence of heavy quarks means that this static dressing can have physical implications. Explicitly we have seen in Sect.'s 6 and 7 how such non-local and non-covariant fields can be used to cure infra-red problems and to derive both a running mass and an inter-quark potential. Such results give us confidence in the approach we have adopted. However, we must consider moving quarks. Thus our proposed identification of the constituent quarks with the dressed quarks in QCD can only hope to be fully successful if we can show how to go beyond this static picture.

From our analysis in Sect. 5, we know that for any acceptable gauge fixing there corresponds a dressing. Thus one possible approach to this problem is to find an appropriate dressing gauge fixing. For the static dressing we have seen that the Coulomb gauge is needed. Now even in this simple situation, there does not seem to be any a priori reason for this connection between the Coulomb gauge and static charges. In Ref. 87 for instance the Coulomb gauge is deemed no more than convenient since then the coupling of transverse gluons to the heavy quarks may be neglected. In Ref. 67 it is again viewed as merely helpful to renormalize the electron propagator at the static point when in Coulomb gauge. (We have seen in Sect. 6 that there is, in fact, really no choice here.) This suggests that, with our present understanding of dressings, it is not fruitful to approach the form for dynamical dressings solely in terms of the type of dressing gauge fixing needed.

If we restrict ourselves to dressings that are momentum eigenstates, then another route to their construction is available - we can simply boost the known static dressing. However, as we have seen in some detail in the previous section, such a proposal comes with its own problems. We have argued that the addition of ghosts allows us to avoid 
complications with the action of the Lorentz group on local fields, but we have seen that modifications arise when dealing with charged fields. Also, from the discussion in Sect. 3, we cannot expect the Lorentz group to act unitarily on, for example, a static configuration.

What we propose to do initially is to construct dressings appropriate to slowly moving charges by just naively boosting. This will allow us to arrive at a rather natural proposal for such dressings and to see at first hand what these general arguments about problems with the Lorentz group really mean in practice. Of course, we cannot expect this rather limited class of dynamical dressings to be directly applicable to complicated bound state problems involving light quarks. In this context, an extension to, say, dressings that are angular momentum eigenstates would be very interesting, but this must be considered future work ${ }^{14}$.

As in the previous section, we will deal in the main with boosting abelian charges. For the non-abelian case this corresponds to working only to order $g$. The extension of this construction to quarks at all orders follows the algorithm described for static quarks once the (dynamical) dressing gauge fixing is identified: we will, though, not enter into these details here.

We recall that the static charge is described by

$$
\psi_{\mathrm{c}}(x)=\exp \left(-\frac{i e}{4 \pi} \int d^{3} y \frac{\partial_{i} A_{i}\left(x^{0}, \boldsymbol{y}\right)}{|\boldsymbol{x}-\boldsymbol{y}|}\right) \psi(x) \text {. }
$$

A boost by velocity $-\boldsymbol{v}$ is generated by the Lorentz charge, $i v_{i} M_{0 i}$. Acting on the spinor we have

$$
\left[i v_{i} M_{0 i}, \psi(x)\right]=\left(v_{i} x_{0} \partial_{i}-v_{i} x_{i} \partial_{0}\right) \psi(x)-\frac{i}{2} v_{i} \sigma_{0 i} \psi(x)
$$

So for small $\boldsymbol{v}$ we have

$$
\psi^{\prime}(x)=S(\Lambda) \psi\left(x^{\prime}\right)
$$

where $S(\Lambda)=\left(1-\frac{i}{2} v_{j} \sigma_{0 j}\right)$ and $x^{0}=x^{0}+v_{j} x^{j}, \boldsymbol{x}^{\prime}=\boldsymbol{x}-\boldsymbol{v} x^{0}$. Similarly on the potential we get

$$
A_{i}^{\prime}\left(x^{0}, \boldsymbol{y}\right)=A_{i}\left(x^{0}+v_{j} y^{j}, \boldsymbol{y}^{\prime}\right)+v_{i} A_{0}\left(x^{0}+v_{j} y^{j}, \boldsymbol{y}^{\prime}\right)
$$

\footnotetext{
14 As we have stated earlier, it seems clear that the dressings we are constructing are related to coherent states, indeed their good infra-red structure necessitates this connection. These coherent states are based on creation and annihilation operators, and hence their dynamical restriction to dressing with a definite momentum is not so surprising. In this context, we would expect that the use of generalized coherent states $^{[103]}$ would allow for a more varied dynamical dressing.
} 
where now $\boldsymbol{y}^{\prime}=\boldsymbol{y}-\boldsymbol{v} x^{0}$.

Naively applying these to the charged field (9.1) gives

$$
\psi_{\mathrm{c}}^{\prime}(x)=S(\Lambda) \exp \left(-\frac{i e}{4 \pi} \int d^{3} z \frac{\partial_{i} A_{i}\left(x^{0}+v_{j} z^{j}, \boldsymbol{z}\right)+v_{i} E_{i}\left(x^{0}+v_{j} z^{j}, \boldsymbol{z}\right)}{\left|\boldsymbol{x}^{\prime}-\boldsymbol{y}\right|}\right) \psi\left(x^{\prime}\right) .
$$

This suggests that a charged particle slowly moving with velocity $\boldsymbol{v}$ can be described by the field

$$
\psi_{\mathrm{v}}(x)=\exp \left(i e \frac{\partial_{i} A_{i}+v_{i} E_{i}}{\nabla^{2}}(x)\right) \psi(x)+O\left(\boldsymbol{v}^{2}\right)
$$

Note that in this expression we have chosen to neglect the overall matrix $S(\Lambda)$, and we have identified the static field $\psi_{\mathrm{c}}$ with $\psi_{\mathbf{o}}$. In keeping with our previous notation, we can also write $(9.6)$ as $\psi_{\mathrm{v}}=e^{i v_{\mathrm{v}}} \psi$.

The first thing to note about $\psi_{\mathrm{v}}$ is that it is gauge invariant: we have in effect only added an electric field term, which is in this abelian theory gauge invariant. We can also repeat the argument presented in Sect. 4, and see that the states created by this field will have, in addition to the Coulomb electric field, the magnetic field $\boldsymbol{B}=\boldsymbol{v} \times \boldsymbol{E}$ expected from such a non-relativistic charge, i.e., the correct associated fields up to first order in the velocity. As it stands, we seem to have been quite successful in boosting the static configuration - one might ask where have the problems with the implementation of the Lorentz group gone? Rather than looking at higher orders in $\boldsymbol{v}$ (where problems do arise, but only after much algebra), let us keep to small velocities but look at how we boost two static quarks.

Starting with two space-like separated static charges:

$$
\psi_{\mathrm{c}}\left(x^{0}-v_{i} x^{i}, \boldsymbol{x}+\boldsymbol{v} x^{0}\right) \psi_{\mathrm{c}}\left(x^{0}-v_{i} y^{i}, \boldsymbol{y}+\boldsymbol{v} x^{0}\right)
$$

boosting gives (up to overall $S(\Lambda)$ factors)

$$
\psi_{\mathrm{v}}\left(x^{0}, \boldsymbol{x}\right) \psi_{\mathrm{v}}\left(x^{0}, \boldsymbol{y}\right) .
$$

Now the initial static fields anti-commute, and that Fermi statistics apply is an important part of the constituent quark model. However, the naively boosted charges given in (9.8) do not commute, which is a direct expression of the subtleties associated with Lorentz transformations of charged states. To see this we consider the equal time anticommutator of two such fields:

$$
\begin{aligned}
{\left[\psi_{\mathrm{v}}(x), \psi_{\mathrm{v}}(y)\right]_{+} } & =\left[e^{i v_{\mathrm{v}}(x)}, e^{i v_{\mathrm{v}}(y)}\right] \psi(x) \psi(y) \\
& =\left(1-e^{\left[v_{\mathrm{v}}(x), v_{\mathrm{v}}(y)\right]}\right) \psi_{\mathrm{v}}(x) \psi_{\mathrm{v}}(y)
\end{aligned}
$$


where we have used the Baker-Campbell-Haussdorf relation

$$
e^{A} e^{B}=e^{A+B} e^{\frac{1}{2}[A, B]}, \quad \text { if } \quad[A,[A, B]]=[B,[A, B]]=0,
$$

which holds for the above photonic clouds. Thus, this anticommutator vanishes if, and only if, $\left[v_{\mathrm{v}}(x), v_{\mathrm{v}}(y)\right]=0$. However, a direct calculation shows that this does not happen. In fact we find that for this specific motion we get

$$
\left[v_{\mathrm{v}}(x), v_{\mathrm{v}}(y)\right]=-i v_{i} \int d^{3} z\left(\frac{\partial}{\partial z^{i}}\left(\frac{1}{|\boldsymbol{x}-\boldsymbol{z}|}\right) \frac{1}{|\boldsymbol{y}-\boldsymbol{z}|}-\frac{1}{|\boldsymbol{x}-\boldsymbol{z}|} \frac{\partial}{\partial z^{i}}\left(\frac{1}{|\boldsymbol{y}-\boldsymbol{z}|}\right)\right) .
$$

By inspection, this commutator generally does not vanish. We note, though, that it is, as expected, zero in the static limit and further vanishes for both $x \rightarrow y$ and, more specifically, if $\boldsymbol{v} \cdot \boldsymbol{x} \rightarrow \boldsymbol{v} \cdot \boldsymbol{y}$.

Here we see a direct manifestation of the fact that the Lorentz transformations fail to be implemented unitarily on such charged fields. In deriving (9.8) we have assumed that

$$
\left[i M_{0 i}, \psi_{c}(x) \psi_{c}(y)\right]=\left[i M_{0 i}, \psi_{c}(x)\right] \psi_{c}(y)+\psi_{c}(x)\left[i M_{0 i}, \psi_{c}(y)\right]
$$

However, in deriving this identity various integrations by parts are performed and we are now not free to discard the resulting surface terms. On top of this problem, we know from our discussion in Sect. 8 that additional surface terms are needed to complement the Lorentz transformations in the charged sector. All in all, we see that the correct implementation of the boost for the two charged system (9.7) is far from clear.

Rather that attempt to derive the correct form of the boost in this situation, we assume that they should anti-commute and find a compensating term ${ }^{15}$ that will ensure the anticommutativity of the boosted fields.

As our ansatz for the two charged system we take

$$
C_{\mathrm{v}}(x, y) \psi_{\mathrm{v}}(x) \psi_{\mathrm{v}}(y)
$$

We now demand that this satisfies the Fermi statistic relation

$$
C_{\mathrm{v}}(x, y) \psi_{\mathrm{v}}(x) \psi_{\mathrm{v}}(y)=-C_{\mathrm{v}}(y, x) \psi_{\mathrm{v}}(y) \psi_{\mathrm{v}}(x)
$$

15 A similar problem arises in conformal field theory where a generalized Klein transformation is needed to ensure the correct commutation relations for bosonised fields ${ }^{[104]}$. In is interesting to note the nonlocality in that construction as well. 
The solution to this is found to be

$$
C_{\mathrm{v}}(x, y)=e^{\left(\frac{1}{2}\left[v_{\mathrm{v}}(x), v_{\mathrm{v}}(y)\right]\right)} .
$$

This of course reduces to unity for the cases discussed above where the dressed fields anticommuted. The need for such phase factors can also be seen in systems of charges moving with different velocities. In fact even for a system composed of a static and a moving charge such terms are present. The non-abelian generalisations of these terms may be readily seen to be field dependent.

To summarize what we have shown so far in this section: the dressing for a slowly moving single charged field can be readily written down using the naive action of the Lorentz boost generators. However, the lack of a unitary implementation of the Lorentz group means now that additional terms are needed to enforce the anti-commutativity of such boosted fields. We also expect any attempt to go beyond the small velocity approximation to encounter difficulties when using the naive Lorentz transformations. Note further that from (9.6) we see that the dressing gauge fixing for small velocity is given by

$$
\partial_{i} A_{i}=-v_{i} E_{i}
$$

To conclude this discussion of dynamical dressings, we will write down a description of a relativistically dressed charge. Rather than building this up from the known static configuration, we will take the form (9.6) as a starting point and follow Dirac's original argument and look now for a gauge invariant field which formally creates the electric and magnetic fields appropriate for a relativistically moving charge.

For simplicity, consider the electron at the point $\boldsymbol{y}$ moving with speed $v$ in the $x^{1}$ direction, then we recall that its electric field at the point $\boldsymbol{x}$ is

$$
\boldsymbol{E}(x)=\frac{e}{4 \pi} \frac{1}{\sqrt{1-v^{2}}} \frac{\boldsymbol{x}-\boldsymbol{y}}{\left(\frac{\left(x_{1}-y_{1}\right)^{2}}{1-v^{2}}+\left(x_{2}-y_{2}\right)^{2}+\left(x_{3}-y_{3}\right)^{2}\right)^{\frac{3}{2}}}
$$

It also has a magnetic field given by

$$
\boldsymbol{B}(x)=\boldsymbol{v} \times \boldsymbol{E}(x) .
$$


The dressing of $\psi$ must reproduce these field configurations in much the same way as the static dressing did for the Coulomb field. A short calculation shows that these follow if we take $\psi_{\mathrm{v}}=e^{v_{\mathrm{v}}} \psi$, where now

$$
\begin{aligned}
v_{\mathrm{v}}(x)=-\frac{i e}{4 \pi} & \frac{1}{\sqrt{1-v^{2}}} \\
& \times \int d^{3} z \frac{\left(1-v^{2}\right) \partial_{1} A_{1}\left(x^{0}, \boldsymbol{z}\right)+\partial_{2} A_{2}\left(x^{0}, \boldsymbol{z}\right)+\partial_{3} A_{3}\left(x^{0}, \boldsymbol{z}\right)-v E_{1}\left(x^{0}, \boldsymbol{z}\right)}{\left(\frac{\left(x_{1}-z_{1}\right)^{2}}{1-v^{2}}+\left(x_{2}-z_{2}\right)^{2}+\left(x_{3}-z_{3}\right)^{2}\right)^{\frac{1}{2}}} .
\end{aligned}
$$

It is straightforward to show that this dressed particle is gauge invariant. Thus we have found the relativistic dressing for an electron.

In summary, we have seen that it is possible to construct dressings for moving charges. That they are generally not simple boosts of the static dressing is a consequence of the way Lorentz transformations must be implemented on charged states. Similarly we have seen the need for phase factors when the charged state contains more than one charge. Such terms need to be better understood. The dressing gauge of a moving charge is no longer the Coulomb one. An immediate conjecture is that the propagator for the moving, dressed charges is infra-red finite only if the appropriate mass shell is used. Similar arguments apply to the renormalisation of vertices. 


\section{Conclusions}

We have seen that in QCD the colour quantum number is only well defined upon gauge invariant states. Gauge invariant descriptions of quarks and gluons have been constructed in perturbation theory. We understood that the static interquark potential can be found more efficiently than in other approaches if the dressed quarks are used. The dressed quark propagator was shown to be free of infra-red divergences and effects of the QCD vacuum on this propagator were incorporated. These results support our proposal that constituent quarks can emerge from QCD as dressed Lagrangian quarks. The fact that quarks have never been seen in any experiment finds its expression in a non-perturbative obstruction to constructing the dressing.

Before we review this in more detail and consider the most obvious tasks at hand in this approach to QCD, we would like to summarise the implications of these ideas for the rest of the standard model. We have seen that descriptions of photonic dressings exist for both static ${ }^{[16]}$ and moving electrons. Here we are not faced with a non-perturbative obstruction. In many ways QED offers us a testing ground for understanding the functions of the dressings around (colour) charges. It would, for example, be interesting to study how, from a dressed electron and a dressed positron, one can form the positronium bound state. We should also stress that the fact that $U(1)$ theory can confine in three dimensions is in no way in disagreement with our ability to dress electrons: the lack of well-defined, gauge invariant, asymptotic fields in a theory is a sufficient but not a necessary condition for the theory to confine. The weak interaction poses a different problem: we observe, e.g., the $W$ and $Z$ bosons and yet their interactions come from a non-abelian gauge theory. The Gribov ambiguity can, however, be sidestepped in theories with spontaneous symmetry breaking by fixing the gauge in the scalar matter (Higgs) sector of the theory. We refer to Ref. 9 for further details. This enables us to construct gauge invariant physical fields in such theories, see also Ref. 105 and 106. Clearly at high temperatures, when the symmetry is restored, this construction will, as in QCD, be obstructed by the Gribov ambiguity. The implications of this, particularly for the early universe, remain to be explored. We should point out here that renormalisation group analyses indicate that the electroweak sector is confining at high temperatures ${ }^{[107]}$. In summary the ideas presented in this paper are in good agreement with the phenomenology of the standard model.

Returning to QCD, we have seen that a gauge invariant definition of a quark is possible inside perturbation theory and we have both found explicit solutions and shown how these 
solutions may be extended to higher orders in the coupling constant. Dressings for both static and non-static quarks were given. These gauge invariant quarks have been shown to possess various desirable attributes. For example, the colour charge, which is usually gauge dependent, was demonstrated to be gauge invariant on all locally gauge invariant states. An important consequence of this is that combinations of such dressed quarks may be put together in the usual group theoretical fashion to form hadrons. As well as such essential symmetry properties, the initial field theoretical investigations reported here were highly promising: we have seen that the infra-red divergence of the one-loop fermion propagator is removed by the dressing if and only if a physically motivated mass shell scheme is employed. Furthermore we have been able to incorporate non-perturbative vacuum effects, in the shape of the quark condensate, into the quark propagator in a gauge invariant way. This indicates how a gauge invariant, non-perturbative constituent quark mass may be constructed.

An attractive aspect of this approach is that a non-perturbative construction of constituent quarks is impossible. The gluonic dressing necessary to ensure gauge invariance cannot be fully defined. This is because the dressing can be used to construct a global gauge fixing and the Gribov ambiguity teaches us that such a perfect gauge fixing does not exist in QCD. This breakdown would be a catastrophe in an analogous description of an electron or, say, a $W$ boson since these are observed particles. However, full descriptions of physical, gauge invariant fields are available to us in both abelian theories like QED $^{[16]}$ and in non-abelian theories with spontaneous symmetry breakdown like the Salam-Weinberg model ${ }^{[9,105,106]}$. In QCD on the other hand we have quark and gluon confinement and our inability to form a non-perturbative definition of the dressing fields is a simple characterization of confinement. The moral of this tale is that at short distances, where perturbative physics prevails, it is possible to describe gauge invariant quarks which, as we have argued, have properties normally required of constituent quarks. At larger distances, in the nonperturbative domain, the breakdown tells us that we can only speak of colourless, gauge invariant hadrons. The quest for the confinement scale can be understood as finding where this breakdown takes place. This is, by its very nature a non-perturbative matter.

The general picture of confinement that emerges from the above parallels a more familiar one ${ }^{[17-19]}$ : at one-loop order in perturbation theory the dressing which removes the infra-red divergence of the quark propagator and allows a description of an asymptotic coloured field is relatively simple (essentially that of the one-loop dressed electron propagator), at two loops the dressing is more complicated, as a result of the non-abelian nature 
of the gauge group, and it becomes harder and harder at higher orders. Non-perturbatively there is no dressing in QCD and the infra-red problem for asymptotic quarks or gluons at the end of the day does not permit a cure.

Various results about charged states emerge naturally in the approach followed here. Our description of quarks is necessarily both non-local and non-covariant, which, we once more stress, are expected characteristics of any charged state. These properties manifest themselves in distinct ways in both QED and QCD, and the similarities and differences are instructive. The non-locality is in each theory a reflection of the long range nature of the force. An electric charge is surrounded by an electric field which falls off only as $1 / R$. This slow fall off is known to be responsible ${ }^{[11]}$ for the infra-red problem in QED. Although one can calculate scattering cross-sections in QED without taking the electromagnetic fields which accompany the asymptotic fields properly into account, one cannot properly discuss physical states without them. The situation is more serious in QCD: here the potential between coloured charges grows with distance. This worsens the infra-red behaviour of Chromodynamics and is responsible for the strong interaction confining colour charges into colour singlet hadrons, the residual interactions between which are of a short range nature.

As well as this non-locality, we have seen that charged states respond to Lorentz transformations in an unexpected way. For example, when there is more than one moving charge, phase factors must be introduced to maintain Fermi statistics. In QED these factors are fields independent. This does not appear to be the case in QCD.

We have argued in Sect. 4 against artificially restoring gauge invariance by attaching a string to a charged field. This introduces an unnatural dependence upon the path we give to the string. Similar criticism can be levelled at constructing hadrons by attaching strings between quarks. Additionally, it has been shown by Shabanov ${ }^{[46]}$ that if we take two static electric charges and connect them by a string then this configuration will radiate off until it eventually approaches the state of a static dressed electron-positron pair. The QCD equivalent of this is obviously a very difficult calculation. If the static charges are at short distances, then presumably they will radiate off gluons in a process which may be described by perturbation theory. However, as the gluons get further away from the fermions they will become sensitive to non-perturbative confining forces, although the vicinity of the fermions should still be in the realm of perturbation theory. If the fermions are further apart then one will presumably see the initial string thicken and form a meson. 
The dressed charges allow for a more direct evaluation of the perturbative static quark potential than has previously been the case. We have seen two ways of evaluating the confinement scale from such calculations: one may investigate at which separation of the quarks the hadronic dressing ceases to factorise into constituent parts or, alternatively, one can try to directly observe the onset of the Gribov problem when one moves two quarks away from each other.

There are many other immediate extensions to this work. The perturbative calculation of the one loop vertices and moving propagators for QED is one of the most obvious. Here we have various predictions about the infra-red behaviour of the dressed Green's functions. These are to be found in Sect.'s 7 and 9. Extensions to higher loops in QCD must also be carried out. As we have stressed, the constituent quark picture cannot arise from perturbation theory alone. It has been possible to include vacuum effects in the shape of the quark condensate. Further work is required to understand how gluonic, and in general higher dimensional, condensates can be included. The main question here is how the nonlocality of the gluonic dressing makes itself felt. In a similar vein, it would be interesting to see if and how instanton effects can be incorporated.

Much use is normally made of BRST and anti-BRST invariance in the analysis of the physical states of gauge theories. Such approaches, though, cannot distinguish the superselection sectors labelled by the velocity of a charge (recall our discussion in Sect. 3). In Ref. 13 we introduced a new, non-local, non-covariant symmetry for QED which could isolate the static charged sector of the theory. This symmetry, unlike BRST or anti-BRST, does not leave the boosted, dressed electrons invariant. We thus conjecture that our symmetry is but one representative of a whole class of new symmetries of QED parameterised by the velocities of the charges. The extension of even the 'static symmetry' to QCD is unknown; however, the work presented here suggests that, within perturbation theory, it may be possible.

The central role of the Gribov ambiguity in our account of confinement warrants our recalling that there is a history ${ }^{[108-109]}$ of linking this problem with confinement which extends right back to Gribov's original paper ${ }^{[52]}$. Most recently there have been attempts $^{[110,111]}$ to restrict non-abelian gauge theories to the region unaffected by the Gribov problem, the fundamental modular domain. This approach aims to construct well defined path integrals, as such it does not help us to build a dressing (see our discussion 
of Lagrangian versus dressing gauge fixings in Sect. 5). Such studies argue that this restriction introduces non-perturbative effects which could be responsible for confinement. The most difficult aspect of this idea is that the boundary of the domain contains all the topological information about the physical configuration space - this results in highly non-trivial identifications of various points on the boundary. The influence of these Gribov copies requires further study.

Finally we wish to list some topics where it seems that it might prove profitable to apply our dressed quarks and gluons. Two of the most obvious applications of dressed quarks are jet physics (see articles in Ref. 1) and the heavy quark effective theory (for a recent review see Ref. 112). Multijet events offer us a window upon quarks and gluons. Our picture is of coloured, dressed quarks and gluons exiting from the event vertex. Initially the dressings are factorised but after some time non-perturbative physics tears the dressings apart and separate colour singlet jets are formed, which hadronise and are then detected. In the effective theory the heavy quarks are surrounded by light degrees of freedom, the socalled 'brown muck' — our approach indicates that some of these light degrees of freedom should be associated with the heavy quark so as to give it a gauge invariant definition. It is important to note here that the preeminent role of the heavy quark's velocity in the effective theory has a natural counterpart in the boosted dressings that we have constructed. Both of these applications are highly intriguing.

There is also much interest in QCD at high temperature and densities ${ }^{[1]}$. Here we would expect hadrons to swell and multiquark structures to form. It also appears that there is some phase transition. The Gribov ambiguity does not vanish at finite temperature. This means that even at high temperatures individual quarks and gluons will not be observables. The implications of this are as yet unclear, however, we are reminded of the expected confining behaviour in the high temperature electroweak sector discussed at the start of this section.

Recent experimental data on that old story the pomeron indicate that it is composed of one hard gluon surrounded by soft degrees of freedom ${ }^{[113]}$. Assuming that this data holds up, it is natural to want to describe the hard gluon (where one might hope to apply perturbation theory) in a gauge invariant way. This would necessitate use of our formulae for dressed gluons from Sect. 5 (or some variant upon them using boosted dressings).

Dressing electric charges correctly can also be used to study hadronic electromagnetic mass differences. In QCD sum rules for hadrons with non-vanishing electric charges the 
currents, and hence the results of such sum rules, are not invariant under $\mathrm{U}(1)$ gauge transformations ${ }^{[114]}$. Although this gauge dependence may be neatly removed by connecting the currents in the correlation function by a string, this is not the most physical choice (the commutation relations would imply that the electric field was only non-zero along the string). A carefully chosen Dirac-type dressing seems more physical. It would be interesting to see how much this changed the results of Ref. 114 .

In conclusion in this paper we have developed a gauge invariant, perturbative description of individual quarks and gluons. These we identify with the constituents of hadrons. We have observed an obstruction to the non-perturbative construction of these fields. This we identify with the expected confinement of such coloured particles. The scale at which hadrons are formed is that where it becomes energetically favourable for the dressing of a hadronic state to no longer factorise into a product of the constituent dressings.

Acknowledgements We wish to thank the following for between them encouraging us, giving constructive criticism, supplying references and reading drafts of the manuscript: Emili Bagan, David Broadhurst, Robin Horan, Stephen Huggett, Alex Kalloniatis, Taro Kashiwa, John Klauder, Peter Landshoff, Peter Lévay, G. Münster, Martin Schaden, Karl Schilcher, Sergei Shabanov, Tom Steele, M. Stingl, John C. Taylor, Izumi Tsutsui, Tassos Vladikas and E. Werner. In addition we would like to thank the organisers of the 16th UK Theory Institute for affording us a chance to finish this paper together. MJL received support from the research project CICYT-AEN95-0815. We thank each other's institutes for hospitality.

Note Added Since the completion of this paper we have together with Emili Bagan verified the above conjectured infra-red finiteness and multiplicative renormalisability of the propagator of the dressed, non-static charge defined by (9.18) at the correct mass shell. This has been done both for small ${ }^{[115]}$ and relativistic ${ }^{[116]}$ velocities. 


\section{Appendix A. The static quark to order $g^{3}$}

In this appendix we shall show how the expression (4.12) for the static quark is derived, and how to extend it to order $g^{3}$. The extension to arbitrary order will be clear.

In our description of the physical quarks and gluons, (5.2) and (5.5), we expand $h_{\mathrm{c}}=e^{-v_{\mathrm{c}}}$ in a power series in the coupling $g$ where

$$
v_{\mathrm{c}}=g v_{(1)}+g^{2} v_{(2)}+g^{3} v_{(3)}+\cdots
$$

Then the quarks and gluons are, to these orders,

$$
\begin{aligned}
\psi^{h_{\mathrm{c}}}=(1+ & g v_{(1)}+g^{2}\left(\frac{1}{2} v_{(1)} v_{(1)}+v_{(2)}\right) \\
& \left.+g^{3}\left(\frac{1}{6} v_{(1)} v_{(1)} v_{(1)}+\frac{1}{2} v_{(1)} v_{(2)}+\frac{1}{2} v_{(2)} v_{(1)}+v_{(3)}\right)\right) \psi
\end{aligned}
$$

and

$$
\begin{aligned}
A_{i}^{h_{\mathrm{c}}}=A_{i} & -\partial_{i} v_{(1)} \\
+ & g\left(\left[v_{(1)}, A_{i}\right]+\frac{1}{2}\left[\partial_{i} v_{(1)}, v_{(1)}\right]-\partial_{i} v_{(2)}\right) \\
+ & g^{2}\left(\left[v_{(2)}, A_{i}\right]+\frac{1}{2}\left[v_{(1)},\left[v_{(1)}, A_{i}\right]\right]+\frac{1}{2}\left[\partial_{i} v_{(1)}, v_{(2)}\right]+\frac{1}{2}\left[\partial_{i} v_{(2)}, v_{(1)}\right]\right. \\
& \left.\quad-\frac{1}{6}\left[v_{(1)},\left[v_{(1)}, \partial_{i} v_{(1)}\right]\right]-\partial_{i} v_{(3)}\right)
\end{aligned}
$$

Our task now is to find $v_{(1)}, v_{(2)}$ and $v_{(3)}$. To this end we exploit the fact that for the static quark these must be such that $A_{i}^{h_{\mathrm{c}}}$ is transverse, i.e., $\partial_{i} A_{i}^{h_{\mathrm{c}}}=0$. This means that we must be able to write

$$
A_{i}^{h_{\mathrm{c}}}=\left(\delta_{i j}-\frac{\partial_{i} \partial_{j}}{\nabla^{2}}\right) \Phi_{j},
$$

where to this order in $g$ we have $\Phi_{i}=\Phi_{(0)} i+g \Phi_{(1)} i+g^{2} \Phi_{(2)} i$.

To order $g^{0},($ A.3) and (A.4) give

$$
\begin{aligned}
\left(\delta_{i j}-\frac{\partial_{i} \partial_{j}}{\nabla^{2}}\right) \Phi_{(0) j} & =A_{i}-\partial_{i} v_{(1)} \\
& =\left(\delta_{i j}-\frac{\partial_{i} \partial_{j}}{\nabla^{2}}\right) A_{j}+\frac{\partial_{i} \partial_{j}}{\nabla^{2}} A_{j}-\partial_{i} v_{(1)}
\end{aligned}
$$

from which we deduce that

$$
v_{(1)}=\frac{\partial_{j} A_{j}}{\nabla^{2}},
$$

and hence that $\Phi_{(0)} i=A_{i}$. 
To the next order in $g$ we get from (A.3) and (A.4)

$$
\begin{aligned}
\left(\delta_{i j}-\frac{\partial_{i} \partial_{j}}{\nabla^{2}}\right) \Phi_{(1)}=( & \left.\delta_{i j}-\frac{\partial_{i} \partial_{j}}{\nabla^{2}}\right)\left(\left[v_{(1)}, A_{j}\right]+\frac{1}{2}\left[\partial_{j} v_{(1)}, v_{(1)}\right]\right) \\
& +\frac{\partial_{i} \partial_{j}}{\nabla^{2}}\left(\left[v_{(1)}, A_{j}\right]+\frac{1}{2}\left[\partial_{j} v_{(1)}, v_{(1)}\right]\right)-\partial_{i} v_{(2)}
\end{aligned}
$$

which implies that

$$
v_{(2)}=\frac{\partial_{j}}{\nabla^{2}}\left(\left[v_{(1)}, A_{j}\right]+\frac{1}{2}\left[\partial_{j} v_{(1)}, v_{(1)}\right]\right),
$$

and $\Phi_{(1) j}=\left[v_{(1)}, A_{j}\right]+\frac{1}{2}\left[\partial_{j} v_{(1)}, v_{(1)}\right]$. Substituting (A.6) and (A.8) into (A.2) yields the expression (4.12) for the quark valid to order $g^{2}$. The expressions for $\Phi_{(0)} j$ and $\Phi_{(1)} j$ yield in (A.4) the description (5.7) for the gluon to order $g$.

This argument can clearly be extended to higher order in $g$. In particular, repeating this argument to order $g^{3}$ gives

$$
\begin{aligned}
v_{(3)}=\frac{\partial_{j}}{\nabla^{2}}\left(\left[v_{(2)}, A_{j}\right]+\frac{1}{2}\left[v_{(1)},\left[v_{(1)}, A_{j}\right]\right]\right. & +\frac{1}{2}\left[\partial_{j} v_{(1)}, v_{(2)}\right]+\frac{1}{2}\left[\partial_{j} v_{(2)}, v_{(1)}\right] \\
& \left.-\frac{1}{6}\left[v_{(1)},\left[v_{(1)}, \partial_{j} v_{(1)}\right]\right]\right)
\end{aligned}
$$

from which the static quark to order $g^{3}$ can be constructed. 


\section{References}

[1] For a recent review of very many aspects and the status of QCD see, QCD - 20 Years later, ed.'s P.M. Zerwas and H.A. Kastrup (World Scientific, two volumes, Singapore, 1993).

[2] M. Gell-Mann and Y. Ne'eman, The Eightfold Way, (W. Benjamin, New York, 1964).

[3] J.J.J. Kokkedee, The Quark Model, (W. Benjamin, New York, 1969).

[4] M. Gell-Mann, Caltech preprint CTSL-26, 1961, (unpublished) reprinted in Ref. 2.

[5] J.I. Friedman, H.W. Kendall and R.E. Taylor, in Nobel Lectures in Physics 1981-1990, ed. G. Ekspong (World Scientific, Singapore 1993).

[6] D. Gross and F. Wilczek, Phys. Rev. Lett. 26 (1973) 1343;

H.D. Politzer, Phys. Rev. Lett. 26 (1973) 1346.

[7] F. Strocchi and A.S. Wightman, J. Math. Phys. 15 (1974) 2198.

[8] M. Lavelle and D. McMullan, Dublin and Mainz preprint DIAS-STP-93-04 and MZ-TH/93-03 (unpublished); Phys. Lett. 329B (1994) 68.

[9] M. Lavelle and D. McMullan, Phys. Lett. 347B (1995) 89.

[10] J.M. Jauch and F. Rohrlich, The Theory of Photons and Electrons, Second Expanded Edition, (Springer-Verlag, New York, 1980).

[11] J.D. Dollard, J. Math. Phys. 5 (1964) 729.

[12] P. Kulish and L. Faddeev, Theor. Math. Phys. 4 (1970) 745.

[13] M. Lavelle and D. McMullan, Phys. Rev. Lett. 71 (1993) 3758.

[14] M. Lavelle and D. McMullan, Phys. Lett. 312B (1993) 211.

[15] P.A.M. Dirac, Canad. J. Phys. 33(1955) 650.

[16] P.A.M. Dirac, The Principles of Quantum Mechanics, (Oxford University Press, 1958).

[17] S. Weinberg, Phys. Rev. Lett. 31 (1973) 494.

[18] D.J. Gross and F. Wilczek, Phys. Rev. D8 (1973) 3633.

[19] H. Fritzsch, M. Gell-Mann and H. Leutwyler, Phys. Lett. 47B (1973) 269.

[20] E. d'Emilio and M. Mintchev, Fortschr. d. Phys. 32 (1984) 473, 503.

[21] O. Steinmann, Ann. Phys. 157 (1984) 232; Helv. Phys. Acta. 58 (1985) 232.

[22] S.V. Shabanov, Mod. Phys. Lett. A6 (1991) 909; Phys. Lett. 255B (1991) 398;

L.V. Prokhorov and S.V. Shabanov, Int. J. Mod. Phys. A7 (1992) 7815.

[23] F.A. Lunev, Mod. Phys. Lett. A9 (1994) 2281.

[24] G. Mack, Fortschr. d. Phys. 29 (1981) 135.

[25] A. Khvedelidze and V. Pervushin, Helv. Phys. Acta. 6 (1994) 610.

[26] Y. Neeman and D. Sijacki, CERN-TH-7461-94.

[27] T. Kugo and I. Ojima, Prog. Theor. Phys. Suppl. No. 66 (1979) 1.

[28] B. Povh et al., Particles and Nuclei, (Springer-Verlag, Berlin, Heidelberg, 1995).

[29] S. Gasiorowicz and J.L. Rosner, Am. J. Phys. 49 (1981) 954.

[30] D. Flamm and F. Schöberl, Introduction to the Quark Model of Elementary Particles Vol. 1, (Gordon and Breach, New York, 1982).

[31] J. Gasser and H. Leutwyler, Nucl. Phys. B94 (1975) 269.

[32] L. Montanet et al., Review of Particle Properties, Phys. Rev. D50 (1994) 1173. 
[33] For the introduction of colour see M. Gell-Mann in Ref. 1.

[34] R. Jackiw, Topological Investigations of Quantized Gauged Theories, in Current Algebra and Anomalies, ed.'s S.B. Treiman et al. (Princeton University Press, Princeton, 1985).

[35] N. Nakanishi and I. Ojima, Covariant Operator Formalism of Gauge Theories and Quantum Gravity (World Scientific, Singapore, 1990).

[36] M. Henneaux and C. Teitelboim, Quantization of Gauge Systems (Princeton University Press, Princeton, New Jersey, 1992).

[37] R. Haag, Local Quantum Physics, (Springer-Verlag, Berlin, Heidelberg, 1993).

[38] For further arguments in this direction see, D. Maison and D. Zwanziger, Nucl. Phys. B91 (1975) 425 and references therein.

[39] R. Haag and D. Kastler, J. Math. Phys. 5 (1964) 848.

[40] G. Roepstorff, Commun. Math. Phys. 19 (1970) 301.

[41] J. Fröhlich, G. Morchio and F. Strocchi, Ann. Phys. 119 (1979) 241.

[42] J.L. Gervais and D. Zwanziger, Phys. Lett. 94B (1980) 389.

[43] D. Buchholz, Phys. Lett. 174B (1986) 331.

[44] J. Polonyi, Phys. Lett. 213B (1988) 340.

[45] D. McMullan and I. Tsutsui, Ann. Phys. 237 (1995) 269.

[46] S.V. Shabanov, Dubna preprint JINR-E2-92-136 (unpublished).

[47] M. Lavelle and D. McMullan, Z. Phys. C59 (1993) 351.

[48] L.D. Faddeev, in Methods in Field Theory, ed.'s R. Balian and J. Zinn-Justin (North-Holland/World Scientific, 1981).

[49] I.M. Singer, Commun. Math. Phys. 60 (1978) 7.

[50] P.K. Mitter and C.M. Viallet, Commun. Math. Phys. 79 (1981) 457.

[51] J. Fuchs, M.G. Schmidt and C. Schweigert, Nucl. Phys. B425 (1994) 107.

[52] V.N. Gribov, Nucl. Phys. B139 (1978) 1.

[53] Encyclopedic Dictionary of Mathematics, ed.'s S. Iyanaga and Y. Kawada (MIT Press, Cambridge, 1977).

[54] See, e.g., B. Hatfield, Quantum Field Theory of Point Particles and Strings, (Addison-Wesley, Redwood City, 1992).

[55] D. Zwanziger, Phys. Rev. D11 (1975) 3481.

[56] V. Chung, Phys. Rev. B140 (1965) 1110.

[57] T.W.B. Kibble, Phys. Rev. 173 (1968) 1527; 174 (1968) 1882; empty175681624.

[58] J.K. Storrow, Nuovo Cimento 54 (1968) 15; 57 (1968) 763.

[59] See, e.g., Chap. 6 of T. Muta, Foundations of Quantum Chromodynamics, (World Scientific, Singapore, 1987).

[60] B. Schroer, Fortschr. d. Phys. 173 (1963) 1527.

[61] H.M. Fried and D.R. Yennie, Phys. Rev. 112 (1958) 1391.

[62] D.J. Broadhurst, N. Gray and K. Schilcher, Z. Phys. C48 (1991) 111.

[63] J.C. Breckenridge, M.J. Lavelle and T.G. Steele, Z. Phys. C65 (1995) 155.

[64] K. Johnson, Ann. Phys. 10 (1960) 536.

[65] R. Hagen, Phys. Rev. 130 (1963) 813.

[66] D. Heckathorn, Nucl. Phys. B156 (1979) 328. 
[67] G.S. Adkins, Phys. Rev. D27 (1983) 1814.

[68] For an overview see: Physical and Nonstandard Gauges, ed.'s P. Gaigg et al, Springer Lecture Notes in Physics 361 (Springer, Heidelberg, 1990).

[69] K.G. Wilson, Phys. Rev. 179 (1969) 1499.

[70] M. Shifman, A. Vainshtein and V. Zakharov, Nucl. Phys. B120 (1977) 385; 448; 519.

[71] H.D. Politzer, Nucl. Phys. B117 (1976) 397.

[72] E. Bagan and T.G. Steele, Phys. Lett. 226B (1989) 142.

[73] P. Pascual and E. de Rafael, Z. Phys. C12 (1982) 12.

[74] M. Lavelle and M. Oleszczuk, Mod. Phys. Lett. A7 (1992) 3617.

[75] J.C. Collins, Renormalisation, (Cambridge University Press, Cambridge, 1984).

[76] M. Lavelle and M. Oleszczuk, Mod. Phys. Lett. A275 (1992) 133.

[77] M. Stingl, Münster preprint MS-TPI-94-13;

U. Häbel et al, Z. Phys. A336 (1990) 423, 435.

[78] V. Elias and M.D. Scadron, Phys. Rev. D30 (1984) 647.

[79] M. Lavelle and M. Schaden, in Ref. 68.

[80] D.J. Broadhurst et al, Phys. Lett. 329B (1994) 103.

[81] K.G. Wilson, Phys. Rev. D10 (1974) 2445.

[82] W. Fischler, Nucl. Phys. B129 (1977) 157.

[83] E.S. Fradkin and G.A. Vilkovisky, Phys. Lett. 55B (1975) 224.

[84] M. Lavelle and D. McMullan, Int. J. Mod. Phys. A7 (1992) 5245.

[85] L.S. Brown and W.I. Weisberger, Phys. Rev. D20 (1979) 3239.

[86] D. Gromes in Phys. Rep. 200C (1991) 127.

[87] T. Appelquist, M. Dine and I.J. Muzinich, Phys. Lett. 69B (1977) 231; Phys. Rev. D17 (1978) 2074.

[88] See, e.g., G. Cella et al, Nucl. Phys. (Proc. Suppl.) B42 (1995) 228.

[89] K. Cahill and D.R. Stump, Phys. Rev. D20 (1979) 2096.

[90] C. Parrinello et al, Phys. Lett. 268B (1991) 236.

[91] M.W. Hecht et al, Phys. Rev. D47 (1992) 285.

[92] See M.L. Paciello et al, Phys. Lett. 341B (1994) 187 and ref.'s therein.

[93] P. de Forcrand and J.E. Hetrick, Nucl. Phys. (Proc. Suppl.) B42 (1995) 861.

[94] K. Cahill and D.R. Stump, Phys. Rev. D20 (1979) 540.

[95] See, e.g, J. Kamesberger et al, Few Body Systems, Suppl. 2, (1987) 529.

[96] M. Tanimoto, Phys. Lett. 116B (1982) 198.

[97] S. Ishida et al, Phys. Rev. D47 (1992) 179.

[98] Y. Ohnuki, Unitary Representations of the Poincaré Group and Relativistic Wave Equations (World Scientific, Singapore, 1988).

[99] R. Jackiw, Field Theoretic Investigations in Current Algebra, in Current Algebra and Anomalies, ed.'s S.B. Treiman et al. (Princeton University Press, Princeton, 1985).

[100] A.O. Barut and R. Raczka, Theory of Group Representations and Applications (Polish Sci., Warsaw, 1977).

[101] R. Jackiw, Phys. Rev. Lett. 41 (1978) 1635. 
[102] R. Jackiw and N.S. Manton, Ann. Phys. 127 (1980) 257.

[103] W. Zhang, D.H. Feng and R. Gilmore, Rev. Mod. Phys. 62 (1990) 867.

[104] P. Goddard and D. Olive, Int. J. Mod. Phys. A1 (1986) 303.

[105] G. 'tHooft, Cargese Lectures 1979.

[106] J. Fröhlich, G. Morchio and F. Strocchi, Phys. Lett. 97B (1980) 249; Nucl. Phys. B190 (1981) 553.

[107] B. Bergerhoff and C. Wetterich, Nucl. Phys. B440 (1995) 171; M. Reuter and C. Wetterich, Nucl. Phys. B408 (1993) 91.

[108] C.M. Bender, T. Eguchi and H. Pagels, Phys. Rev. D17 (1978) 1086.

[109] R.D. Peccei, Phys. Rev. D17 (1978) 1097.

[110] P. van Baal, Nucl. Phys. B369 (1992) 259.

[111] D. Zwanziger, Nucl. Phys. B345 (1990) 461, M. Schaden and D. Zwanziger, New York preprint NYU-ThPhSZ94-1.

[112] M. Neubert, Phys. Rep. 245C (1994) 259.

[113] R. Ball, private communication.

[114] L.S. Kisslinger and Z. Li, Phys. Rev. Lett. 74 (1995) 2168.

[115] E. Bagan, M. Lavelle and D. McMullan, Barcelona/Plymouth preprint UAB-FT-379/PLY-MS-95-08, to appear in Physics Letters B.

[116] E. Bagan, M. Lavelle and D. McMullan, Barcelona/Plymouth preprint UAB-FT-384/PLY-MS-96-01, submitted for publication. 
This figure "fig1-1.png" is available in "png" format from: http://arxiv.org/ps/hep-ph/9509344v2 